\title{
Parameter Degeneracy in Neutrino Oscillation — Solution Network and Structural Overview -
}

\author{
Hisakazu Minakataf and Shoichi Uchinamit \\ Department of Physics, Tokyo Metropolitan University \\ 1-1 Minami-Osawa, Hachioji, Tokyo 192-0397, Japan
}

(Dated: April 27, 2010)

\begin{abstract}
It is known that there is a phenomenon called "parameter degeneracy" in neutrino oscillation measurement of lepton mixing parameters; A set of the oscillation probabilities, e.g., $P\left(\nu_{\mu} \rightarrow \nu_{e}\right)$ and its CP-conjugate $P\left(\bar{\nu}_{\mu} \rightarrow \bar{\nu}_{e}\right)$ at a particular neutrino energy does not determine uniquely the values of $\theta_{13}$ and $\delta$. With use of the approximate form of the oscillation probability á la Cervera et al., a complete analysis of the eightfold parameter degeneracy is presented. We propose a unified view of the various types of the degeneracy as invariance of the oscillation probabilities under discrete mappings of the mixing parameters. Explicit form of the mapping is obtained either by symmetry argument, or by deriving exact analytic expressions of all the degeneracy solutions for a given true solution. Due to the one-to-one mapping structure the degeneracy solutions are shown to form a network. We extend our analysis into the parameter degeneracy in T- and CPT-conjugate measurement as well as to the setup with the golden and the silver channels, $P\left(\nu_{e} \rightarrow \nu_{\mu}\right)$ and $P\left(\nu_{e} \rightarrow \nu_{\tau}\right)$. Some characteristic features of the degeneracy solutions in CPconjugate measurement, in particular their energy dependences, are illuminated by utilizing the explicit analytic solutions.
\end{abstract}

PACS numbers:

*Electronic address: minakata@tmu.ac.jp

$\dagger$ Electronic address: uchinami@phys.metro-u.ac.jp 


\section{INTRODUCTION}

After establishing the neutrino masses and the lepton flavor mixing [1] by the atmospheric [2], the solar [3], and the reactor experiments [4], which is further supported by the accelerator experiments [5, 6], there seems to exist a consensus that the next step is to measure $\theta_{13}$ and $\delta$, the remaining unknowns in the MNS matrix, and to determine the neutrino mass hierarchy. It was proposed that if $\theta_{13}$ is relatively large an intense neutrino beam from nuclear reactors can be used to measure it by using the near-far two-detector setting [7, 8. Alternatively, or complimentarily, the accelerator search for nonzero $\theta_{13}$ has advantage of potential possibility of extending it to $\mathrm{CP}$ violation search. The reactor $9-12$ and the accelerator experiments [13, 14] are either ongoing or in construction to look for effects of nonzero $\theta_{13}$.

It is well known, however, that detection of $\mathrm{CP}$ violation due to the lepton KobayashiMaskawa phase $\delta$ [15], being the genuine three flavor effect, is suppressed by the two small factors, the ratio $\Delta m_{21}^{2} / \Delta m_{31}^{2}\left[5,6,16-18\right.$ ] and the value of $\theta_{13}$ bounded from above [19 22]. Therefore, high precision experiments are inevitably required to measure CP violating phase $\delta$. Once precision measurement becomes the necessity, the experiment is better characterized as a simultaneous determination of $\theta_{13}$ and $\delta$. It is because even though one enjoys prior crude knowledges of magnitude of $\theta_{13}$ (assuming it relatively large), which certainly propels $\mathrm{CP}$ measurement, the required precision for detecting tiny effects of $\delta$ necessitates simultaneous measurement of $\theta_{13}$ in a precision far beyond the previously achieved ones.

It is known that a set of measurement of the oscillation probabilities, e.g., $P\left(\nu_{\mu} \rightarrow \nu_{e}\right)$ and its CP conjugate $P\left(\bar{\nu}_{\mu} \rightarrow \bar{\nu}_{e}\right)$ at a particular neutrino energy, no matter how accurate, does not determine uniquely the values of $\theta_{13}$ and $\delta$, the problem of parameter degeneracy [23 25]. The nature of the degeneracy can be understood as the so called intrinsic degeneracy [23] duplicated by the unknown sign of $\Delta m_{31}^{2}$ [24] and $\theta_{23}$ octant [25], which entails the total eightfold degeneracy if $\theta_{23} \neq \pi / 4$. The feature can be seen in Fig. 1. Some features of the degeneracy were further discussed in [27 29], whose first two noticed special features that appear in the vacuum oscillation maximum. The notorious feature of the degeneracy is that difference between the true and the fake solutions can be so small that their distinction is extremely difficult, rendering resolution of completely different physical pictures, e.g., the mass hierarchies, untenable. Or, in the other cases, the difference between the true and the fake values of $\delta$ is so large to confuse $\mathrm{CP}$ violation with $\mathrm{CP}$ conservation.

It is the purpose of this paper to give a complete analysis of the parameter degeneracy in neutrino oscillations. To achieve a unified understanding of the phenomenon, we present and advance a new view of the degeneracy as an invariance of the oscillation probabilities under discrete mappings of the mixing parameters. With use of the approximate form of the oscillation probability obtained in [30] we present a self-contained derivation (rederivation in CP conjugate case) of the analytic expressions of all the degeneracy solutions as functions of the true solutions, which supplies the explicit form of the mapping. Having the analytic solutions of the eightfold degeneracy with the proper convention at hand, we demonstrate that they form a solution network, the one-to-one correspondence structure between solutions, which will be pictorially represented in Fig. 4 in Sec. V.

We use the analytic solutions to make plots of the differences between the true and the fake solutions to illuminate the global overview of the degeneracy. We illuminate, by using the plots, the characteristic features of the degeneracy and reveal the reasons why and how the sign- $\Delta m_{31}^{2}$ and the $\theta_{23}$ octant degeneracies are robust against the spectrum analysis. 

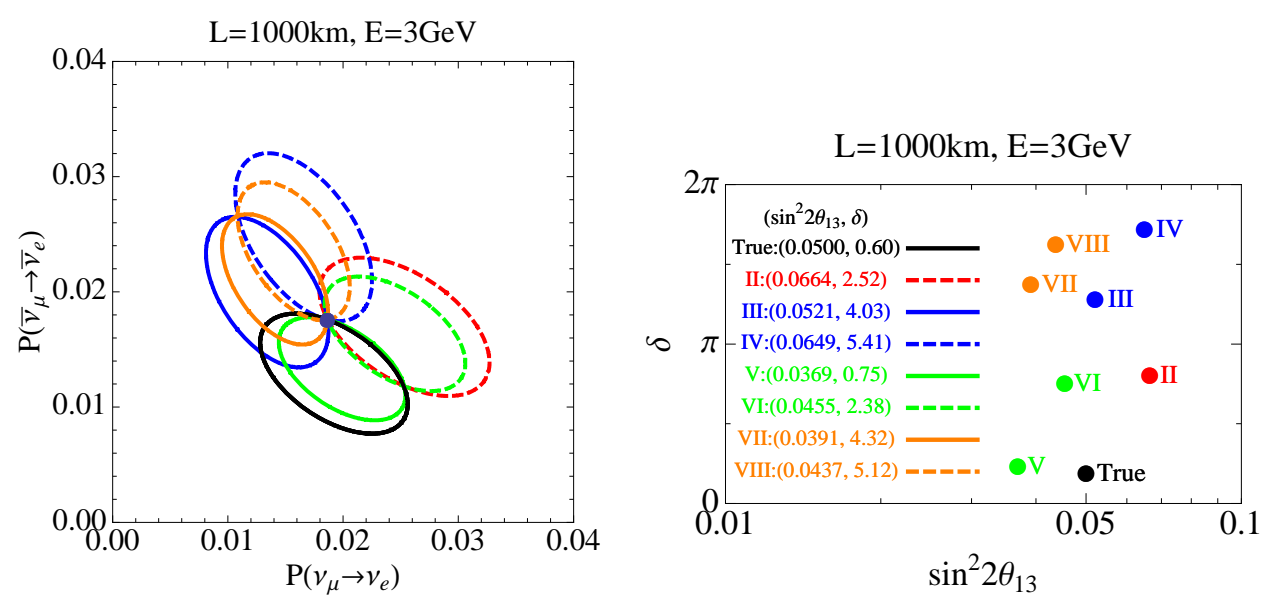

FIG. 1: Left panel: An illustrative example of the eightfold degeneracy is represented pictorially (as first appeared in [26]) in terms of the bi-probability plot in $P-P^{C P}$ space [24]. Right panel: The parameters $\left(\sin ^{2} 2 \theta_{13}, \delta\right)$ of the true solutions and the clone ones II-VIII are presented as numbers and also by the dots in $\sin ^{2} 2 \theta_{13}-\delta$ space. The correspondence between the ellipses and the solution labels are made manifest by using the same color lines/symbols in both panels.

We note that the first attempt toward analytic solutions of the degeneracy was pursuit by the authors of [27] who obtained the solution for the intrinsic degeneracy. Then, the similar analysis was extended in [31] to include the degeneracy solutions which involve the $\Delta m_{31}^{2}$-sign and/or the $\theta_{23}$ octant flips.

One may ask; Why is the parameter degeneracy defined as above way so relevant? Mathematically speaking, the degeneracy is easy to solve; Repeating measurement at one more energy (or baseline), or adding a different oscillation channel immediately solves degeneracy. Or, if the spectrum information is available it may be more powerful to resolve the degeneracy. Despite these valid reasonings, unfortunately, at least some type of the degeneracy is shown to be robust and survives in varying experimental settings. One of the reasons for it is, as we will see in Sec. VI, that the energy dependence of difference between the true and the fake solutions is so mild that spectrum information is not powerful enough to resolve the degeneracy.

We emphasize that need for resolution of the degeneracy is not only because precision measurement is always desirable, but also because, far more importantly, it leaves e.g., the neutrino mass hierarchy undetermined after huge experimental efforts. We want to warn the readers that we will not try to discuss how the degeneracy can be lifted by assuming concrete experimental settings. ${ }^{1}$ Rather, we focus in on a complete understanding of structure of the degeneracy. The explicit analytic solutions and knowledges of structure of the degeneracy should serve for clearer understanding of the experimental data taken in precision measurement in the future. It will be definitely called for if future neutrino experiments reveal features that may not fit in into the standard three-neutrino mixing to clearly discriminate

1 The analysis presented in this paper may be regarded as "pathological analysis of neutrino oscillation". It would not tell us directly the experimental method for solving the degeneracy, but as in the case of pathology of human body, the clearer understanding of the disease may ultimately provide with us the correct recipe for its resoluion. 
confusion by the degeneracy from new effects beyond the standard three-flavor mixing. For example, if they are so powerful to detect neutrino's nonstandard interactions (NSI) [32 36] (see [37] for further references therein), the event structure will be modified by the new ingredients and enriched with new type of the degeneracies [38, 39]. For the importance of lifting the degeneracy, quite naturally, a great amount of efforts were devoted to investigate how it can be done. The references [40 64] are nothing but only a small subset of them.

In the next section, we define our machinery and introduce a new view of the degeneracy as invariance under the discrete transformations. Then, in the following two sections (III) and IV), we first give a complete treatment of the parameter degeneracy with CP-conjugate measurement. In Sec. V, we present the explicit form of the discrete mapping and complete our understanding of the structure of the degeneracy. It will be supplemented by the discussions of degeneracy in T-conjugate (Sec. VII), the golden-silver (Sec. VIII), and CPTconjugate (Sec. IX) measurement combining the $\nu_{e} \rightarrow \nu_{\mu}$ (golden) and the $\nu_{e} \rightarrow \nu_{\tau}$ (silver) channels.

\section{NEUTRINO OSCILLATION PROBABILITY AND ITS INVARIANCE}

In our analysis in this paper, we rely on the approximate formula for the appearance oscillation probability derived by Cervera et al. [30]. A simple way of deriving the formula is to use perturbative framework in which $s_{13}$ is assumed to be of order $\frac{\Delta m_{21}^{2}}{\Delta m_{31}^{2}} \equiv \epsilon$ and keep the terms up to second order in $\epsilon$. For a review of this method, see e.g., [38]. Here, $\Delta m_{j i}^{2} \equiv m_{j}^{2}-m_{i}^{2}(i, j=1,2,3)$. However, in this paper, we take an attitude to utilize the formula as far as it is reasonably accurate, even outside the region of validity of the perturbative ansatz. In fact, it is known that the formula gives a reasonable description of the oscillation probability even with larger values of $s_{13}$ [65].

\section{A. Approximate formula of the neutrino oscillation probability}

To present the formula for the $\nu_{e}$ appearance probability in a compact way we use the simplified notations. We summarize them together with their magnitudes for convenience of the readers:

$$
\begin{aligned}
s & \equiv s_{13} \\
\Delta_{j i} & \equiv\left|\frac{\Delta m_{j i}^{2} L}{4 E}\right|=1.27\left(\frac{\left|\Delta m_{j i}^{2}\right|}{10^{-3} \mathrm{eV}^{2}}\right)\left(\frac{L}{1000 \mathrm{~km}}\right)\left(\frac{E}{1 \mathrm{GeV}}\right)^{-1} \quad(i, j=1,2,3), \\
A & \equiv \frac{a L}{4 E}=0.27\left(\frac{\rho}{2.8 \mathrm{~g} / \mathrm{cm}^{3}}\right)\left(\frac{L}{1000 \mathrm{~km}}\right)
\end{aligned}
$$

where $a \equiv 2 \sqrt{2} G_{F} N_{e} E$, the well known coefficient related to the index of refraction of neutrinos in matter [32]. $G_{F}$ is the Fermi constant, $\rho$ and $N_{e} \equiv \rho / m_{N}$ with $m_{N}$ being the nucleon mass denote, respectively, the averaged matter and the electron number densities along the neutrino trajectory in the earth, and we have assumed that the electron fraction $Y_{e}$ is 0.5 . By using the definition of $\Delta_{j i}$ as positive definite quantities we choose to display explicitly the sign of $\Delta m_{31}^{2}$ as \pm signs (sometimes called as the hierarchy signs) in the equations. 
It may be useful to remember the ratio between the vacuum and the matter parameters for understanding the feature of the degeneracy solutions in Sec. VI.

$$
\frac{A}{\Delta_{31}}=0.085\left(\frac{\left|\Delta m_{j i}^{2}\right|}{2.5 \times 10^{-3} \mathrm{eV}^{2}}\right)^{-1}\left(\frac{\rho}{2.8 \mathrm{~g} / \mathrm{cm}^{3}}\right)\left(\frac{E}{1 \mathrm{GeV}}\right) .
$$

Therefore, in typical low-energy superbeam [66]68] experiments the ratio is small, $A / \Delta_{31} \lesssim$ 0.1 , whereas in neutrino factory [69, 70] with baseline of several thousand kilometers the ratio is large, $A / \Delta_{31} \sim 3-6$.

The oscillation probabilities of the neutrino flavor conversion processes $\nu_{\mu} \rightarrow \nu_{e}$, its CPconjugate channel $\bar{\nu}_{\mu} \rightarrow \bar{\nu}_{e}$, the T-conjugate channel $\nu_{e} \rightarrow \nu_{\mu}$, and the CPT-conjugate channel $\bar{\nu}_{e} \rightarrow \bar{\nu}_{\mu}$, in matter are given under the constant matter density approximation as 30

$$
\begin{aligned}
P \equiv P\left(\nu_{\mu} \rightarrow \nu_{e}\right) & =X_{ \pm} s^{2}+Y_{ \pm} s \cos \left(\delta \pm \Delta_{31}\right)+Z \\
P^{C P} \equiv C P\left[P\left(\nu_{\mu} \rightarrow \nu_{e}\right)\right] & =P\left(\bar{\nu}_{\mu} \rightarrow \bar{\nu}_{e}\right)=\bar{X}_{ \pm} s^{2}+\bar{Y}_{ \pm} s \cos \left(\delta \mp \Delta_{31}\right)+Z \\
& =X_{\mp} s^{2}-Y_{\mp} s \cos \left(\delta \mp \Delta_{31}\right)+Z \\
P^{T} \equiv T\left[P\left(\nu_{\mu} \rightarrow \nu_{e}\right)\right] & =P\left(\nu_{e} \rightarrow \nu_{\mu}\right)=X_{ \pm} s^{2}+Y_{ \pm} s \cos \left(\delta \mp \Delta_{31}\right)+Z \\
P^{C P T} \equiv C P T\left[P\left(\nu_{\mu} \rightarrow \nu_{e}\right)\right] & =P\left(\bar{\nu}_{e} \rightarrow \bar{\nu}_{\mu}\right)=\bar{X}_{ \pm} s^{2}+\bar{Y}_{ \pm} s \cos \left(\delta \pm \Delta_{31}\right)+Z \\
& =X_{\mp} s^{2}-Y_{\mp} s \cos \left(\delta \pm \Delta_{31}\right)+Z
\end{aligned}
$$

where \pm indicates the mass hierarchy, namely, the normal and the inverted mass hierarchies for the positive and the negative $\Delta m_{31}^{2}$, respectively. The functions $X_{ \pm}, Y_{ \pm}$, and $Z$ are defined by

$$
\begin{aligned}
X_{ \pm} & =4 s_{23}^{2}\left[\frac{\Delta_{31} \sin \left(\Delta_{31} \mp A\right)}{\left(\Delta_{31} \mp A\right)}\right]^{2}, \\
Y_{ \pm} & = \pm 2 \sqrt{X_{ \pm} P_{\odot}}= \pm 4 \sin 2 \theta_{12} c_{23} s_{23}\left[\frac{\Delta_{31} \sin \left(\Delta_{31} \mp A\right)}{\left.\Delta_{31} \mp A\right)}\right]\left[\frac{\Delta_{21} \sin A}{A}\right] \\
Z & =c_{23}^{2} \sin ^{2} 2 \theta_{12}\left[\frac{\Delta_{21} \sin A}{A}\right]^{2} .
\end{aligned}
$$

Their forms imply that the oscillation probability can be written as $P=\mid \sqrt{X} s+$ $\left.\mathrm{e}^{i\left(\delta \pm \Delta_{31}\right)} \sqrt{Z}\right|^{2}$, which allows simple interpretation of the $\delta$-sensitive term as an interference between the atmospheric and the solar scale oscillations. $\bar{X}$ and $\bar{Y}$ in (4) and (6) are related to $X$ and $Y$ as

$$
\begin{gathered}
\bar{X}_{ \pm}(a)=X_{ \pm}(-a)=X_{\mp}(a) \\
\bar{Y}_{ \pm}(a)=Y_{ \pm}(-a)=-Y_{\mp}(a) .
\end{gathered}
$$

In our discussions in this paper, it is crucial to note the relation [29] between the coefficients $X_{ \pm}$and $Y_{ \pm}$:

$$
\frac{Y_{+}}{\sqrt{X_{+}}}=-\frac{Y_{-}}{\sqrt{X_{-}}}
$$

which follows from the definitions. Notice that (8) means that the same relation as (9) holds also for $\bar{X}$ and $\bar{Y}$. 
In this paper, our emphasis is placed on the oscillation channels between $\nu_{\mu}$ and $\nu_{e}$ and their anti-particles. To have a clearer view of the structure of parameter degeneracy, however, we will include the $\nu_{e} \rightarrow \nu_{\tau}$ appearance channel, which is sometimes called the "silver channel" [71]. See Sec. VIII. The oscillation probability $P\left(\nu_{e} \rightarrow \nu_{\tau}\right)$ is given by

$$
P^{S} \equiv P\left(\nu_{e} \rightarrow \nu_{\tau}\right)=\cot ^{2} \theta_{23} X_{ \pm} s^{2}-Y_{ \pm} s \cos \left(\delta \mp \Delta_{31}\right)+\tan ^{2} \theta_{23} Z .
$$

\section{B. Parameter degeneracy as an invariance of the oscillation probability under discrete mapping}

It is not so well recognized that the appearance oscillation probability in matter under the Cervera et al. approximation has an invariance. Namely, it is easy to show that the oscillation probabilities $P, P^{T}$, and $P^{S}$ defined in (3), (5) and (10), respectively, with positive $\Delta m_{31}^{2}$ is invariant under the transformation

$$
\begin{aligned}
\Delta m_{31}^{2} & \rightarrow-\Delta m_{31}^{2}, \\
s & \rightarrow \sqrt{\frac{X_{+}}{X_{-}}} s, \\
\delta & \rightarrow \pi-\delta .
\end{aligned}
$$

Notice that the first transformation transforms $X_{+}$and $Y_{+}$into $X_{-}$and $Y_{-}$, respectively, and use has been made of the key relation (9). Similarly, the oscillation probability with negative $\Delta m_{31}^{2}$ has an invariance similar to 11 , replacing the second one with $s \rightarrow \sqrt{\frac{X_{-}}{X_{+}}} s$. It is nothing but a generalization of the invariance of the oscillation probability in vacuum to that in matter, which was used to show the existence of degeneracy solutions with differing sign of $\Delta m_{31}^{2}[24$.

Then, it immediately follows that there exists the sign- $\Delta m_{31}^{2}$ degeneracy in measurement which combines any two of $P, P^{T}$, and $P^{S}$, and the explicit form of the degenerate solution can be obtained by the symmetry alone. Later in Sec. VII and Sec. VIII we will explicitly verify it by working out explicit solutions.

A better understanding of the implication of the invariance may be achieved by drawing the bi-probability plot [24]. Here, we take the particular one in $P-P^{T}$ space [72] as shown in Fig. 2. The simultaneous invariance of $P=P\left(\nu_{\mu} \rightarrow \nu_{e}\right)$ and $P^{T}=P\left(\nu_{e} \rightarrow \nu_{\mu}\right)$ for any values of $\theta_{13}$ and $\delta$ means that one can find always two completely overlapping ellipses, one with positive and the other negative $\Delta m_{31}^{2}$. The $\delta$ label, if placed onto the ellipses, are different between the two ellipses, and they are related by $\delta_{-}=\pi-\delta_{+}$, where $\delta_{ \pm}$denote the $\delta$ for the respective hierarchies. More comments on the degeneracy in T-conjugate measurement will follow in Sec. VII.

Now, let us focus on the degeneracy with CP-conjugate measurement. Unfortunately, the similar simple-minded symmetry argument does not go through in the settings with CP and CPT conjugate measurement; The positive $\Delta m_{31}^{2} \mathrm{CP}$ and CPT conjugate probabilities $P^{C P}$ and $P^{C P T}$ defined in (4) and (6) are not simultaneously invariant under (11). That is, $P^{C P}$ and $P^{C P T}$ are invariant under a transformation $s \rightarrow \sqrt{\bar{X}_{+} / \bar{X}_{-}} s$ accompanied with the other transformations in (11). But, the transformation cannot be identical with (11) in matter. It is also true that the similar symmetry argument does not go through for the intrinsic and the $\theta_{23}$ octant degeneracies. 


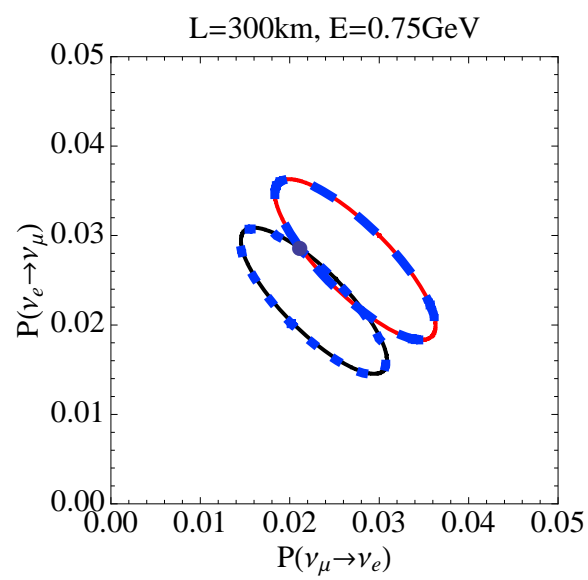

FIG. 2: $P-P^{T}$ bi-probability plot. $P-P^{T}$ bi-probability plot by using the approximate formulas given in (3) and (5). $\sin ^{2} 2 \theta_{13}=0.05$ and $\mathrm{NH}$ (black solid), 0.061 and $\mathrm{NH}$ (red solid), 0.058 and IH (blue dotted), 0.069 and IH (blue dashed).

However, we will show by the discussions throughout the following two sections and Sec. IX that there is a similar invariance of a pair of the oscillation probabilities, e.g., $P$ and $P^{C P}$ in the $\mathrm{CP}$-conjugate measurement, under the transformation

$$
\begin{aligned}
s_{1} & \rightarrow s_{\mathrm{N}}=\xi_{\mathrm{N}}\left(s_{1}, \delta_{1}, \theta_{23}^{\text {true }}\right), \\
\delta_{1} & \rightarrow \delta_{\mathrm{N}}=\eta_{\mathrm{N}}\left(s_{1}, \delta_{1}, \theta_{23}^{\text {true }}\right) \\
\Delta m_{31}^{2} & \rightarrow+\Delta m_{31}^{2}, \text { or }-\Delta m_{31}^{2} \\
\theta_{23}^{\text {true }} & \rightarrow \theta_{23}^{\text {true }}, \text { or } \theta_{23}^{\text {false }}
\end{aligned}
$$

In (12), $s_{1}, \delta_{1}$, and $\theta_{23}^{\text {true }}$ is the true parameters with subscript " 1 ", the unique case with Arabic numerals. The degeneracy solutions are labeled by using Roman subscripts $\mathrm{N}=\mathrm{II}-\mathrm{VIII}{ }^{2}$ Alternative choices in the last two transformations determine whether the degeneracy is of the type involving the sign change of $\Delta m_{31}^{2}$, or the $\theta_{23}$ octant flip. In this sense, the parameter degeneracy is nothing but the statement of invariance of $P$ and $P^{C P}$ under the discrete transformation (12). Furthermore, what is to be really stressed is that the mapping in (12) can be constructed by the basic three mappings. See Sec. V.

\section{INTRINSIC AND SIGN- $\Delta m_{31}^{2}$ DEGENERACIES; CP-CONJUGATE MEA- SUREMENT}

\section{A. Preliminary remarks}

Since this is the first section to actually solve the degeneracy problem to obtain the clone solutions we make some preliminary remarks. In the rest of this paper, we analyze the

2 We note that each type of degeneracy is only two-fold merely because of the approximate form of the probabilities we use, or in other word, due to the smallness of $\theta_{13}$. Existence of more solutions may be signaled e.g., by the $s_{13}^{3}$ terms in the oscillation probabilities in a large $-\theta_{13}$ perturbation theory [73]. 
structure of parameter degeneracy from various viewpoints, in particular, explicit analytic solutions, the symmetry aspect (as already mentioned), and use of the bi-probability plot to illuminate the respective characteristic features of the degeneracy. While the solutions were presented in a condensed way in [31], we present a step-by-step derivation of the degeneracy solutions because it is reader friendly and makes the understanding of structure of degeneracy much easier. We emphasize that the analytic solution with the proper convention for its definition is the integral part of our discussion of solution network to be given in Sec. $\mathrm{V}$.

In most part of this paper, we confine ourselves into the oscillation channels between $\nu_{\mu}$ and $\nu_{e}$ and their antiparticles. In particular, we focus on $\nu_{e}$ (and $\bar{\nu}_{e}$ ) appearance channel which will be available in conventional $\nu_{\mu}$ superbeam in this and the next sections. On the other hand, the T-conjugate channels, $\nu_{e} \rightarrow \nu_{\mu}$ and $\bar{\nu}_{e} \rightarrow \bar{\nu}_{\mu}$, would be provided by neutrino factory and the beta beam [74, 75]. Given understanding the degeneracy of the former channels, the corresponding informations on the latter may be obtained by regarding $\delta$ by $2 \pi-\delta$. We include $\nu_{\tau}$ appearance channel in Sec. VIII.

In this paper we take the method for obtaining the degeneracy solutions for a given set of true parameters $\left(s_{1}, \delta_{1}\right)$. Equivalently, one can choose an alternative way of obtaining the degeneracy solutions as a function of "observable", e.g., $P$ and $P^{C P}$, as pursued in [29]. If one want to take this attitude, one can simply do it (at least numerically) by regarding that both the clone solutions and $\left(P, P^{C P}\right)$ are parametrically represented by $\left(s_{1}, \delta_{1}\right)$.

Notational comment: We denote the mass hierarchy of the true solution by the \pm signs (+ for the normal and - for the inverted) to make the hierarchy choice always explicit. The relationship between the degeneracy solutions with input true mass hierarchies will be further discussed in Sec. V.

Now, let us start our discussion of parameter degeneracy by taking CP-conjugate measurement. The setting seems to be the most promising one experimentally in the near future. In this section we confine ourselves to the degeneracy solutions which have the same $\theta_{23}$ octant, though we treat generically the case of arbitrary values of $\theta_{23}$. The degeneracy solutions across octants of $\theta_{23}$ will be discussed in the next section.

\section{B. The intrinsic degeneracy in CP-conjugate measurement}

With expression of the oscillation probabilities in (3) and (4), the intrinsic degeneracy solutions $\left(s_{i}, \delta_{i}\right)(\mathrm{i}=1,2)$ in $\mathrm{CP}$-conjugate measurement are defined with $\nu_{\mu} \rightarrow \nu_{e}$ channel by

$$
\begin{aligned}
& P-Z=X_{ \pm} s_{1}^{2}+Y_{ \pm} s_{1}\left(\cos \delta_{1} \cos \Delta_{31} \mp \sin \delta_{1} \sin \Delta_{31}\right), \\
& P-Z=X_{ \pm} s_{2}^{2}+Y_{ \pm} s_{2}\left(\cos \delta_{2} \cos \Delta_{31} \mp \sin \delta_{2} \sin \Delta_{31}\right),
\end{aligned}
$$

and in CP-conjugate channel by

$$
\begin{aligned}
& P^{C P}-Z=X_{\mp} s_{1}^{2}-Y_{\mp} s_{1}\left(\cos \delta_{1} \cos \Delta_{31} \pm \sin \delta_{1} \sin \Delta_{31}\right), \\
& P^{C P}-Z=X_{\mp} s_{2}^{2}-Y_{\mp} s_{2}\left(\cos \delta_{2} \cos \Delta_{31} \pm \sin \delta_{2} \sin \Delta_{31}\right) .
\end{aligned}
$$

By subtracting two equations in (13) and (14) respectively, we obtain

$$
\begin{aligned}
& \left(s_{1}^{2}-s_{2}^{2}\right)+\frac{Y_{ \pm}}{X_{ \pm}} \cos \Delta_{31}\left(s_{1} \cos \delta_{1}-s_{2} \cos \delta_{2}\right) \mp \frac{Y_{ \pm}}{X_{ \pm}} \sin \Delta_{31}\left(s_{1} \sin \delta_{1}-s_{2} \sin \delta_{2}\right)=0, \\
& \left(s_{1}^{2}-s_{2}^{2}\right)-\frac{Y_{\mp}}{X_{\mp}} \cos \Delta_{31}\left(s_{1} \cos \delta_{1}-s_{2} \cos \delta_{2}\right) \mp \frac{Y_{\mp}}{X_{\mp}} \sin \Delta_{31}\left(s_{1} \sin \delta_{1}-s_{2} \sin \delta_{2}\right)=0 .
\end{aligned}
$$


From (15) we can obtain the expressions of $\cos \delta_{2}$ and $\sin \delta_{2}$ as

$$
\begin{aligned}
& s_{2} \cos \delta_{2}=s_{1} \cos \delta_{1} \pm \frac{2}{\cos \Delta_{31}} \frac{C^{(-)}}{\left(C^{(+)}\right)^{2}-\left(C^{(-)}\right)^{2}}\left(s_{2}^{2}-s_{1}^{2}\right) \\
& s_{2} \sin \delta_{2}=s_{1} \sin \delta_{1} \pm \frac{2}{\sin \Delta_{31}} \frac{C^{(+)}}{\left(C^{(+)}\right)^{2}-\left(C^{(-)}\right)^{2}}\left(s_{2}^{2}-s_{1}^{2}\right)
\end{aligned}
$$

where $C^{( \pm)}$is defined by

$$
C^{( \pm)} \equiv \frac{Y_{+}}{X_{+}} \pm \frac{Y_{-}}{X_{-}}
$$

Inserting (16) into $\cos ^{2} \delta_{2}+\sin ^{2} \delta_{2}=1$ gives a quartic equation for $s_{2}$ as $\left(s_{2}^{2}-s_{1}^{2}\right)\left(s_{2}^{2}-s_{\mathrm{II}}^{2}\right)=$ 0 . Of course, we obtain the trivial solution $s_{2}=s_{1}$, the situation unique to discussions of the intrinsic degeneracy. The genuine intrinsic degeneracy solution is given by

$$
\begin{aligned}
s_{\text {II }}=\left[s_{1}^{2} \pm \frac{1}{\left(1+R^{2}\right)}\left(s_{1} \cos \delta_{1}\right.\right. & \left.+R s_{1} \sin \delta_{1}\right)\left(C^{(-)} \cos \Delta_{31}-R C^{(+)} \sin \Delta_{31}\right) \\
& \left.+\frac{1}{4\left(1+R^{2}\right)}\left(C^{(-)} \cos \Delta_{31}-R C^{(+)} \sin \Delta_{31}\right)^{2}\right]^{1 / 2}
\end{aligned}
$$

where $R$ is defined by ${ }^{3}$

$$
R \equiv \frac{C^{(+)}}{C^{(-)}} \cot \Delta_{31}
$$

Notice that the $\pm \operatorname{sign}$ in (18) represents the mass hierarchy of the true solution. By using (18) into (16) we obtain the solution of $\delta_{\mathrm{II}}$. From (16) one can obtain the following expressions:

$$
\begin{aligned}
s_{\mathrm{II}} \sin \left(\delta_{1}+\delta_{\mathrm{II}}\right) & =-\frac{2 R s_{1}}{1+R^{2}} \mp \frac{1}{2\left(1+R^{2}\right)}\left(\sin \delta_{1}+R \cos \delta_{1}\right)\left(C^{(-)} \cos \Delta_{31}-R C^{(+)} \sin \Delta_{31}\right), \\
s_{\mathrm{II}} \cos \left(\delta_{1}+\delta_{\mathrm{II}}\right) & =-\left(\frac{1-R^{2}}{1+R^{2}}\right) s_{1} \\
& \mp \frac{1}{2\left(1+R^{2}\right)}\left(\cos \delta_{1}-R \sin \delta_{1}\right)\left(C^{(-)} \cos \Delta_{31}-R C^{(+)} \sin \Delta_{31}\right) .
\end{aligned}
$$

If we further expand the solution in 18 by the solar-atmospheric ratio $\frac{\Delta m_{21}^{2}}{\Delta m_{31}^{2}}$, we obtain

$$
s_{\mathrm{II}}=s_{1}+\frac{s_{1}}{2\left(1+R^{2}\right)}\left(\cos \delta_{1}+R \sin \delta_{1}\right)\left(C^{(-)} \cos \Delta_{31}-R C^{(+)} \sin \Delta_{31}\right) .
$$

Similarly, the equation (20) also simplifies to $\cos \left(\delta_{1}+\delta_{2}\right)=-\left(1-R^{2}\right) /\left(1+R^{2}\right)$. These expressions reproduce the ones derived in [23, 29].

3 We note the relationship between the notations in this paper and in the previous papers [23, 29], denoted here as MNP: $\left.C^{( \pm)}\right|_{\text {this }}=\left.2 C^{(\mp)}\right|_{\text {MNP }}$, and $\left.R\right|_{\text {this }}=\left.z^{-1}\right|_{\text {MNP }}$. 


\section{The sign- $\Delta m^{2}$ degeneracy in CP-conjugate measurement}

We turn to the flipped $\Delta m^{2}$-sign degeneracy in CP-conjugate measurement. The true input solution $\left(s_{1}, \delta_{1}\right)$ and the opposite sign clone solution $\left(s_{3}, \delta_{3}\right)$ satisfy the following equations. In the neutrino channel,

$$
\begin{aligned}
& P-Z=X_{ \pm} s_{1}^{2}+Y_{ \pm} s_{1}\left(\cos \delta_{1} \cos \Delta_{31} \mp \sin \delta_{1} \sin \Delta_{31}\right), \\
& P-Z=X_{\mp} s_{3}^{2}+Y_{\mp} s_{3}\left(\cos \delta_{3} \cos \Delta_{31} \pm \sin \delta_{3} \sin \Delta_{31}\right),
\end{aligned}
$$

and in CP-conjugate channel

$$
\begin{aligned}
& P^{C P}-Z=X_{\mp} s_{1}^{2}-Y_{\mp} s_{1}\left(\cos \delta_{1} \cos \Delta_{31} \pm \sin \delta_{1} \sin \Delta_{31}\right), \\
& P^{C P}-Z=X_{ \pm} s_{3}^{2}-Y_{ \pm} s_{3}\left(\cos \delta_{3} \cos \Delta_{31} \mp \sin \delta_{3} \sin \Delta_{31}\right),
\end{aligned}
$$

By combining the first and the second equations in (22) and (23) we obtain

$$
\begin{aligned}
& \frac{X_{ \pm}}{X_{\mp}} s_{1}^{2}-s_{3}^{2}+\cos \Delta_{31}\left(\frac{Y_{ \pm}}{X_{\mp}} s_{1} \cos \delta_{1}-\frac{Y_{\mp}}{X_{\mp}} s_{3} \cos \delta_{3}\right) \\
& \mp \sin \Delta_{31}\left(\frac{Y_{ \pm}}{X_{\mp}} s_{1} \sin \delta_{1}+\frac{Y_{\mp}}{X_{\mp}} s_{3} \sin \delta_{3}\right)=0, \\
& \frac{X_{\mp}}{X_{ \pm}} s_{1}^{2}-s_{3}^{2}-\cos \Delta_{31}\left(\frac{Y_{\mp}}{X_{ \pm}} s_{1} \cos \delta_{1}-\frac{Y_{ \pm}}{X_{ \pm}} s_{3} \cos \delta_{3}\right) \\
& \mp \sin \Delta_{31}\left(\frac{Y_{\mp}}{X_{ \pm}} s_{1} \sin \delta_{1}+\frac{Y_{ \pm}}{X_{ \pm}} s_{3} \sin \delta_{3}\right)=0 .
\end{aligned}
$$

Using $C^{( \pm)}$defined in 17 we can simplify the equations. By subtracting and adding two equations in (24) we obtain

$$
\begin{array}{r}
T_{1 \pm}^{C P}-C^{(+)} s_{3} \cos \delta_{3} \cos \Delta_{31}+C^{(-)} s_{3} \sin \delta_{3} \sin \Delta_{31}=0, \\
T_{2 \pm}^{C P}-2 s_{3}^{2} \pm C^{(-)} s_{3} \cos \delta_{3} \cos \Delta_{31} \mp C^{(+)} s_{3} \sin \delta_{3} \sin \Delta_{31}=0,
\end{array}
$$

In $(25), T_{1 \pm}^{C P}$ and $T_{1 \pm}^{C P}$ are defined as:

$$
\begin{aligned}
& T_{1 \pm}^{C P} \equiv \pm E^{(-)} s_{1}^{2}+D^{(+)} s_{1} \cos \delta_{1} \cos \Delta_{31}-D^{(-)} s_{1} \sin \delta_{1} \sin \Delta_{31}, \\
& T_{2 \pm}^{C P} \equiv E^{(+)} s_{1}^{2} \pm D^{(-)} s_{1} \cos \delta_{1} \cos \Delta_{31} \mp D^{(+)} s_{1} \sin \delta_{1} \sin \Delta_{31},
\end{aligned}
$$

where we have introduced the new notations $D^{( \pm)}$and $E^{( \pm)}$as

$$
D^{( \pm)} \equiv \frac{Y_{+}}{X_{-}} \pm \frac{Y_{-}}{X_{+}}, \quad E^{( \pm)} \equiv \frac{X_{+}}{X_{-}} \pm \frac{X_{-}}{X_{+}}
$$

From (25) we obtain the expressions of $\cos \delta_{3}$ and $\sin \delta_{3}$ as

$$
\begin{aligned}
& s_{3} \cos \delta_{3}=\frac{1}{\cos \Delta_{31}} \frac{1}{\left(C^{(+)}\right)^{2}-\left(C^{(-)}\right)^{2}}\left[C^{(+)} T_{1 \pm}^{C P} \pm C^{(-)}\left(T_{2 \pm}^{C P}-2 s_{3}^{2}\right)\right], \\
& s_{3} \sin \delta_{3}=\frac{1}{\sin \Delta_{31}} \frac{1}{\left(C^{(+)}\right)^{2}-\left(C^{(-)}\right)^{2}}\left[C^{(-)} T_{1 \pm}^{C P} \pm C^{(+)}\left(T_{2 \pm}^{C P}-2 s_{3}^{2}\right)\right] .
\end{aligned}
$$


We insert (28) into $\cos ^{2} \delta_{3}+\sin ^{2} \delta_{3}=1$ we obtain the quartic equation for $s_{3}$ as

$$
4\left(1+R^{2}\right) s_{3}^{4}-4 U_{ \pm} s_{3}^{2}+V_{ \pm}=0
$$

where

$$
\begin{gathered}
U_{ \pm} \equiv \frac{1}{4} \cos ^{2} \Delta_{31}\left(C^{(-)}\right)^{2}\left[1-\left(\frac{C^{(+)}}{C^{(-)}}\right)^{2}\right]^{2} \pm \frac{1}{\sin ^{2} \Delta_{31}}\left(\frac{C^{(+)}}{C^{(-)}}\right) T_{1 \pm}^{C P}+\left(1+R^{2}\right) T_{2 \pm}^{C P} \\
V_{ \pm} \equiv\left[\cot ^{2} \Delta_{31}+\left(\frac{C^{(+)}}{C^{(-)}}\right)^{2}\right]\left(T_{1 \pm}^{C P}\right)^{2} \pm \frac{2}{\sin ^{2} \Delta_{31}}\left(\frac{C^{(+)}}{C^{(-)}}\right) T_{1 \pm}^{C P} T_{2 \pm}^{C P}+\left(1+R^{2}\right)\left(T_{2 \pm}^{C P}\right)^{2}
\end{gathered}
$$

Notice that $R$ is defined in (19). Equation (29) has the obvious solutions

$$
s_{3}^{2}=\frac{1}{2\left(1+R^{2}\right)}\left[U_{ \pm}[ \pm]^{*} \sqrt{U_{ \pm}^{2}-\left(1+R^{2}\right) V_{ \pm}}\right]
$$

where $[ \pm]^{*}$ denotes a temporary sign which is independent of the hierarchy sign. We discuss immediately below (Sec. IIID) the way how to determine the sign convention. One can easily show by using Cauchy-Schwarz inequality that $V_{ \pm} \geq 0$, and hence the right-hand-side of (32) is non-negative. Therefore, there are four real solutions of $s_{3}$ and the two positive ones are physical; The sign- $\Delta m_{31}^{2}$ degeneracy is two-fold.

The region specified by $D_{ \pm}^{\text {sign }} \equiv U_{ \pm}^{2}-\left(1+R^{2}\right) V_{ \pm} \leq 0$ defines the region in which there is no sign- $\Delta m_{31}^{2}$ degeneracy solution. The region of no solution is displayed in Fig. 3 by taking the three typical setups for superbeam type experiments, SB1, MB1, and MB2 settings, which will be defined in Sec. VIA ${ }^{4}$ In this figure the true mass hierarchy is taken to be the inverted one. If we take the input normal hierarchy we must have the figure with $\delta$ shifted by $\pi$, as one can confirm by looking at the white region in Figs. 10 and 12 in Sec. VIC. This is expected by the general discussion to be given in Sec. VB.

A notable feature is that the region of absent solution occupies mostly around $\delta \sim \pi / 2$ in the left two panels in Fig. 3 where $L / E$ taken are at around the first oscillation maximum. The region is farthest to the "central region" populated by the both $\Delta m_{31}^{2}$-sign ellipses in the bi-probability space, and hence it is the region of lucky resolution of the sign- $\Delta m_{31}^{2}$ degeneracy [76] for the inverted $(\delta \sim 3 \pi / 2$ for the normal) mass hierarchy. Generally speaking the no sign-degeneracy region grows for longer baseline, and the tendency continues to e.g., $L=4000 \mathrm{~km}$ and $E=20 \mathrm{GeV}$ (Figs. 18 and 19 in Sec. VIE). However, the feature changes for a region of the second oscillation maximum as seen in the third panel in Fig. 3 . Because of the dynamic behavior of the bi-probability ellipses (see e.g., Fig. 2 in [53]) there are much better chance of having the sign- $\Delta m_{31}^{2}$ degeneracy.

\section{Problem of convention of labeling the degenerate solution}

We denote the two solutions in (32) as $s_{\mathrm{III}}$ and $s_{\mathrm{IV}}$. It is a highly nontrivial issue how to define these two solutions. In principle there are two ways:

4 Apparently, a complete description of the no solution region of the sign- $\Delta m_{31}^{2}$ and the $\theta_{23}$ octant degeneracies seems to be lacking in the literature. 

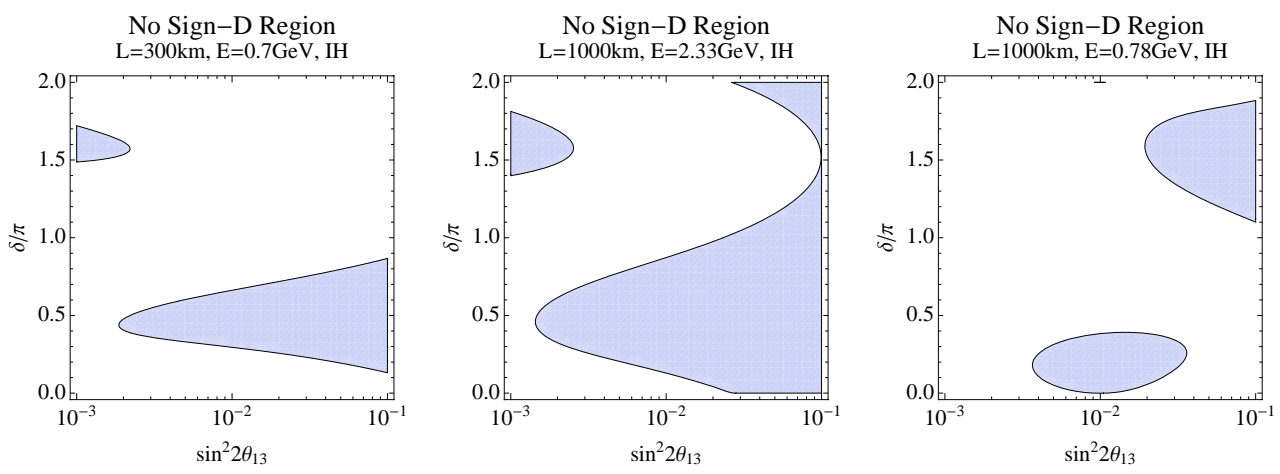

FIG. 3: Depicted as the shaded areas in the $\sin ^{2} 2 \theta_{13}-\delta / \pi$ space are the regions where no sign$\Delta m_{31}^{2}$ degeneracy solution exists for the same three set of the baselines and the neutrino energies as in Figs. 10 and 12. The true mass hierarchy is taken to be the inverted one, and it may help to understand the relationship between the degeneracy solutions with different true mass hierarchies to be discussed in Sec. VB.

Convention A: One can take the convention such that always $s_{\mathrm{IV}} \geq s_{\mathrm{III}}$. That is, the plus and the minus signs in $(32)$ correspond to $s_{\mathrm{IV}}$ and $s_{\mathrm{III}}$, respectively.

Convention B: One may choose the other convention such that the vacuum limit of the degenerate solutions can be taken smoothly.

For reasons explained below we adopt the convention B. We note that $D_{ \pm}^{\text {sign }}$ defined as $D_{ \pm}^{\text {sign }} \equiv U_{ \pm}^{2}-\left(1+R^{2}\right) V_{ \pm}$can be written as

$$
\begin{aligned}
& D_{ \pm}^{\text {sign }}=\left\{1-\left(\frac{C^{(+)}}{C^{(-)}}\right)^{2}\right\}^{2}\left[\frac{1}{16} \cos ^{4} \Delta_{31}\left(C^{(-)}\right)^{4}\left\{1-\left(\frac{C^{(+)}}{C^{(-)}}\right)^{2}\right\}^{2}\right. \\
& \left.-\cot ^{2} \Delta_{31}\left(T_{1 \pm}^{C P}\right)^{2} \pm \frac{1}{2} \cot ^{2} \Delta_{31} C^{(+)} C^{(-)} T_{1 \pm}^{C P}+\frac{1}{2} \cos ^{2} \Delta_{31}\left(C^{(-)}\right)^{2}\left(1+R^{2}\right) T_{2 \pm}^{C P}\right] .
\end{aligned}
$$

In the vacuum oscillation limit, $a \rightarrow 0, C^{(+)}, D^{(+)}, R$, and $E^{(-)}$all vanish, and $E^{(+)}=2$ and $D^{(-)}=C^{(-)}=C_{\text {vac }}^{(-)}$hold, where

$$
C_{\mathrm{vac}}^{(-)}=\lim _{a \rightarrow 0} \frac{2 Y_{+}}{X_{ \pm}}=\frac{2 \Delta_{21}}{\sin \Delta_{31}} \sin 2 \theta_{12} \cot \theta_{23}
$$

Then, $D_{ \pm}^{\text {sign }}$ has a vacuum limit $D_{ \pm}^{\text {oct-vac }} \equiv \lim _{a \rightarrow 0} D_{ \pm}^{\text {sign }} \equiv\left(d_{ \pm}^{\text {sign }}\right)^{2}$ where

$$
d_{ \pm}^{\text {sign }}=C_{\mathrm{vac}}^{(-)} \cos \Delta_{31}\left(\frac{1}{4} C_{\mathrm{vac}}^{(-)} \cos \Delta_{31} \pm s_{1} \cos \delta_{1}\right) \text {. }
$$

The smooth limit to the sign- $\Delta m_{31}^{2}$ degenerate solution in vacuum can be achieved by taking the sign convention

$$
\begin{gathered}
s_{\mathrm{III}}^{2}=\frac{1}{2\left(1+R^{2}\right)}\left[U_{ \pm}-d_{ \pm}^{\text {sign }} \sqrt{\frac{D_{ \pm}^{\text {sign }}}{\left(d_{ \pm}^{\text {sign }}\right)^{2}}}\right] \\
s_{\mathrm{IV}}^{2}=\frac{1}{2\left(1+R^{2}\right)}\left[U_{ \pm}+d_{ \pm}^{\text {sign }} \sqrt{\frac{D_{ \pm}^{\text {sign }}}{\left(d_{ \pm}^{\text {sign }}\right)^{2}}}\right] .
\end{gathered}
$$


In (36) we have taken the convention such that in $a \rightarrow 0$ limit $s_{\text {III }}$ and $s_{\text {IV }}$ smoothly tend to $s_{\mathrm{III}}^{\mathrm{vac}}$ and $s_{\mathrm{IV}}^{\mathrm{vac}}$, respectively, in vacuum defined in Sec. VIID. Once the solutions of $s_{3}$ are specified with the well defined convention the solutions $\delta_{\mathrm{III}}$ and $\delta_{\mathrm{IV}}$ can be obtained by inserting $s_{\mathrm{III}}$ and $s_{\mathrm{IV}}$, respectively, into (28).

We take the convention $\mathrm{B}$ because of number of desirable features. The matter perturbation theory [77, 78] can be formulated only with the convention because it requires the existence of smooth limit $a \rightarrow 0$ in each solution. More importantly, the convention B makes the structure of the degenerate solutions transparent. That is, if we denote $s_{\text {III }}$ in (36) and $\delta_{\text {III }}$ in 28 in an abstract fashion as

$$
s_{\mathrm{III}}=\xi_{ \pm}^{\mathrm{CP} \operatorname{sign}}\left(s_{1}, \delta_{1}\right), \quad \delta_{\mathrm{III}}=\eta_{ \pm}^{\mathrm{CP} \operatorname{sign}}\left(s_{1}, \delta_{1}\right),
$$

then, one can show that

$$
s_{\mathrm{IV}}=\xi_{ \pm}^{\mathrm{CP} \operatorname{sign}}\left(s_{\mathrm{II}}, \delta_{\mathrm{II}}\right), \quad \delta_{\mathrm{IV}}=\eta_{ \pm}^{\mathrm{CP} \operatorname{sign}}\left(s_{\mathrm{II}}, \delta_{\mathrm{II}}\right) .
$$

In this sense there is the one-to-one correspondence between the two intrinsic degeneracy solutions and the sign- $\Delta m_{31}^{2}$ degeneracy solutions, a charming property which one can enjoy only with the convention B. ${ }^{5}$ The relations will be further extended into the other types pf degeneracies and completed in Sec. V.

However, there exists a somewhat disturbing feature of this convention; The solutions have discontinuity as a function of $E$, the neutrino energy. It by no means, however, that the solutions are unphysical. It merely implies that the two solutions interchange themselves at the discontinuous point.

\section{IV. $\theta_{23}$ OCTANT DEGENERACY IN CP-CONJUGATE MEASUREMENT}

In this section we address the parameter degeneracy solutions, assuming that $\theta_{23} \neq \pi / 4$, which have the different $\theta_{23}$ octant from the true one. We denote the octant in which the quantity lives by the superscript "true" or "false" where the true $\theta_{23}$ can be in either the first or the second octants. Our treatment of the octant degeneracy will be done under the approximation that the two solutions of $\theta_{23}$ has the same value of $\sin 2 \theta_{23}$. In this approximation $Y_{ \pm}$is independent of the octant, but $X_{ \pm}$and $Z$ in (7) have to have additional superscripts such as $X_{ \pm}^{\text {true }}$ or $X_{ \pm}^{\text {false }}$ to indicate in which octant they live. Using the definition of $X_{ \pm}$and $Z_{ \pm}$given in (7) they are related with each other as

$$
X_{ \pm}^{\text {false }}=\cot ^{2} \theta_{23}^{\text {true }} X_{ \pm}^{\text {true }}, \quad Z_{ \pm}^{\text {false }}=\tan ^{2} \theta_{23}^{\text {true }} Z_{ \pm}^{\text {true }}
$$

\section{A. Intrinsic degeneracy in the false $\theta_{23}$ octant}

We first discuss the case in which the intrinsic degeneracy solutions exist in a $\theta_{23}$ octant different from the true one. The input solution $\left(s_{1}, \delta_{1}\right)$ and the different octant clone solution

5 Notice that $s_{\mathrm{II}} \geq s_{1}$, or $s_{\mathrm{II}} \leq s_{1}$, depending upon the region of experimental parameters. Therefore, once the correspondence relations (37) and (38) are established, we cannot take the convention A which implies that always $s_{\mathrm{IV}} \geq s_{\mathrm{III}}$. If we try to solve the problem of obtaining the eightfold degeneracy solutions for a given set of $\left(P, P^{C P}\right)$. it would be possible to take the convention A. 
$\left(s_{5}, \delta_{5}\right)$ satisfy the following equations

$$
\begin{aligned}
& P=X_{ \pm}^{\text {true }} s_{1}^{2}+Y_{ \pm} s_{1}\left(\cos \delta_{1} \cos \Delta_{31} \mp \sin \delta_{1} \sin \Delta_{31}\right)+Z^{\text {true }} \\
& P=X_{ \pm}^{\text {false }} s_{5}^{2}+Y_{ \pm} s_{5}\left(\cos \delta_{5} \cos \Delta_{31} \mp \sin \delta_{5} \sin \Delta_{31}\right)+Z^{\text {false }}
\end{aligned}
$$

in the neutrino channel, and

$$
\begin{aligned}
& P^{C P}=X_{\mp}^{\text {true }} s_{1}^{2}-Y_{\mp} s_{1}\left(\cos \delta_{1} \cos \Delta_{31} \pm \sin \delta_{1} \sin \Delta_{31}\right)+Z^{\text {true }} \\
& P^{C P}=X_{\mp}^{\text {false }} s_{5}^{2}-Y_{\mp} s_{5}\left(\cos \delta_{5} \cos \Delta_{31} \pm \sin \delta_{5} \sin \Delta_{31}\right)+Z^{\text {false }}
\end{aligned}
$$

in the CP-conjugate channel. The way we obtain $s_{5}$ and $\delta_{5}$ follows exactly the one for the sign- $\Delta m_{31}^{2}$ degeneracy solutions in Sec. IIIC. Therefore, we can skip many equations and just say that (40) and (41) lead to

$$
\begin{aligned}
s_{5} \cos \delta_{5} & =\frac{1}{\cos \Delta_{31}} \frac{1}{\left(C_{2}^{(+)}\right)^{2}-\left(C_{2}^{(-)}\right)^{2}}\left[C_{2}^{(+)} T_{3 \pm}^{C P} \mp C_{2}^{(-)}\left(T_{4 \pm}^{C P}-2 s_{5}^{2}\right)\right], \\
s_{5} \sin \delta_{5} & =\frac{1}{\sin \Delta_{31}} \frac{1}{\left(C_{2}^{(+)}\right)^{2}-\left(C_{2}^{(-)}\right)^{2}}\left[C_{2}^{(-)} T_{3 \pm}^{C P} \mp C_{2}^{(+)}\left(T_{4 \pm}^{C P}-2 s_{5}^{2}\right)\right] .
\end{aligned}
$$

In (42), we have used the new notations which essentially is a generalization of the previous one as well as new ones:

$$
\begin{aligned}
C_{2}^{( \pm)} & \equiv \frac{Y_{+}}{X_{+}^{\text {false }}} \pm \frac{Y_{-}}{X_{-}^{\text {false }}}, \quad F^{( \pm)} \equiv \frac{X_{+}^{\text {true }}}{X_{+}^{\text {false }}} \pm \frac{X_{-}^{\text {true }}}{X_{-}^{\text {false }}} \\
G^{( \pm)} & \equiv\left(\frac{1}{X_{+}^{\text {false }}} \pm \frac{1}{X_{-}^{\text {false }}}\right)\left(Z^{\text {true }}-Z^{\text {false }}\right)
\end{aligned}
$$

and defined $T_{3 \pm}^{C P}$ and $T_{4 \pm}^{C P}$, in parallel with $T_{1 \pm}^{C P}$ and $T_{2 \pm}^{C P}$ in $(26)$, as

$$
\begin{aligned}
& T_{3 \pm}^{C P} \equiv \pm F^{(-)} s_{1}^{2} \pm G^{(-)}+C_{2}^{(+)} s_{1} \cos \delta_{1} \cos \Delta_{31}-C_{2}^{(-)} s_{1} \sin \delta_{1} \sin \Delta_{31}, \\
& T_{4 \pm}^{C P} \equiv F^{(+)} s_{1}^{2}+G^{(+)} \pm C_{2}^{(-)} s_{1} \cos \delta_{1} \cos \Delta_{31} \mp C_{2}^{(+)} s_{1} \sin \delta_{1} \sin \Delta_{31} .
\end{aligned}
$$

Inserting (42) into $\cos ^{2} \delta_{5}+\sin ^{2} \delta_{5}=1$ we obtain the quartic equation for $s_{5}$ as

$$
4\left(1+R_{2}^{2}\right) s_{5}^{4}-4 H_{ \pm} s_{5}^{2}+I_{ \pm}=0
$$

which is actually a quadratic equation of $s_{13}^{2}$ because of the quadratic dependence on $s_{13}$ of $s_{5} \cos \delta_{5}$ and $s_{5} \sin \delta_{5}$. Thus, there exist only two physical (i.e., positive) solutions, which implies that the octant degeneracy is two-fold. In (45) $H$ and $I$ are defined as

$$
\begin{aligned}
& H_{ \pm}=\frac{1}{4} \cos ^{2} \Delta_{31}\left(C_{2}^{(-)}\right)^{2}\left\{1-\left(\frac{C_{2}^{(+)}}{C_{2}^{(-)}}\right)^{2}\right\}^{2} \mp \frac{1}{\sin ^{2} \Delta_{31}}\left(\frac{C_{2}^{(+)}}{C_{2}^{(-)}}\right) T_{3 \pm}^{C P}+\left(1+R_{2}^{2}\right) T_{4 \pm}^{C P}, \\
& I_{ \pm}=\left[\cot ^{2} \Delta_{31}+\left(\frac{C_{2}^{(+)}}{C_{2}^{(-)}}\right)^{2}\right]\left(T_{3 \pm}^{C P}\right)^{2} \mp \frac{2}{\sin ^{2} \Delta_{31}}\left(\frac{C_{2}^{(+)}}{C_{2}^{(-)}}\right) T_{3 \pm}^{C P} T_{4 \pm}^{C P}+\left(1+R_{2}^{2}\right)\left(T_{4 \pm}^{C P}\right)^{2} \cdot(47
\end{aligned}
$$


where $R_{2} \equiv \frac{C_{2}^{(+)}}{C_{2}^{(-)}} \cot \Delta_{31}$. Then, the octant degeneracy solution $s_{5}$ is given by

$$
s_{5}^{2}=\frac{1}{2\left(1+R_{2}^{2}\right)}\left[H_{ \pm}[ \pm]^{*} \sqrt{D_{ \pm}^{\text {oct-intr }}}\right],
$$

where $[ \pm]^{*}$ is the temporary sign to be specified below, and $D_{ \pm}^{\text {oct-intr }}$ is defined as

$$
\begin{aligned}
D_{ \pm}^{\text {oct-intr }} & =\left\{1-\left(\frac{C_{2}^{(+)}}{C_{2}^{(-)}}\right)^{2}\right\}^{2}\left[\frac{1}{16} \cos ^{4} \Delta_{31}\left(C_{2}^{(-)}\right)^{4}\left\{1-\left(\frac{C_{2}^{(+)}}{C_{2}^{(-)}}\right)^{2}\right\}^{2}\right. \\
& \left.-\cot ^{2} \Delta_{31}\left(T_{3 \pm}^{C P}\right)^{2} \mp \frac{1}{2} \cot ^{2} \Delta_{31} C_{2}^{(+)} C_{2}^{(-)} T_{3 \pm}^{C P}+\frac{1}{2} \cos ^{2} \Delta_{31}\left(C_{2}^{(-)}\right)^{2}\left(1+R_{2}^{2}\right) T_{4 \pm}^{C P}\right] .
\end{aligned}
$$

The region defined by $D_{ \pm}^{\text {oct-intr }} \leq 0$ defines the region in which there is no intrinsic degeneracy solution in an octant different from the true $\theta_{23}$. Once there is a solution, it must be obvious that $s_{5}^{2}$ is positive definite following the similar argument as in Sec. IIIC.

The region of no $\theta_{23}$ octant degeneracy solution is displayed as the white regions in the top two panels in Figs. 14 and 16 for superbeam type settings, SB1, MB1, and MB2 (to be defined in Sec. VIA). The corresponding informations for neutrino factory setting NF are given in Figs. 18 and 19. In fact, one can observe that the feature of no octant degeneracy region is insensitive to the baseline and energies, but depend on in which $\theta_{23}$ octant the true solution exists. If $\theta_{23}^{\text {true }}$ is in the first octant, no-degeneracy regions are around $\delta \sim \pi / 2$ and $3 \pi / 2$, whereas if it is in the second octant it is confined into the small $\theta_{13}$ region, $\sin ^{2} 2 \theta_{13} \lesssim 10^{-3}$. Both of the features can be easily understood by drawing the bi-probability plot; The region of no degeneracy solution is the one spanned only by the ellipses with a single octant $\theta_{23}$ [79].

Now, we have to revisit the issue of convention to define unambiguously the octant degeneracy solutions. We take the following new convention:

Convention C: We define $\left(s_{\mathrm{V}}, \delta_{\mathrm{V}}\right)$ and $\left(s_{\mathrm{VI}}, \delta_{\mathrm{VI}}\right)$ such that they have a smooth limit to the intrinsic degeneracy solutions $\left(s_{1}, \delta_{1}\right)$ and $\left(s_{\mathrm{II}}, \delta_{\mathrm{II}}\right)$, respectively, when the maximum $\theta_{23}$ limit $\theta_{23} \rightarrow \pi / 4$ is taken. It can be understood as a consistency condition.

The convention allows us to formulate perturbative framework with use of the small expansion parameter $\theta_{23}-\pi / 4$, as we will do in Appendix B. Notice that the degenerate solutions $\left(s_{\mathrm{II}}, \delta_{\mathrm{II}}\right),\left(s_{\mathrm{III}}, \delta_{\mathrm{III}}\right)$, and $\left(s_{\mathrm{IV}}, \delta_{\mathrm{IV}}\right)$ themselves obtained in Sec. III are valid independent of the value of $\theta_{23}$.

In the maximum $\theta_{23}$ limit, $F^{(-)}$and $G^{( \pm)}$all vanish, and $F^{(+)}=2, C_{2}^{( \pm)}=C^{( \pm)}$and $R_{2}=R$ hold. Then, $D_{ \pm}^{\text {oct-intr }}$ has the maximum $\theta_{23}$ limit

$$
D_{ \pm}^{\text {oct-intr-max }} \equiv \lim _{\theta_{23} \rightarrow \pi / 4} D_{ \pm}^{\text {oct-intr }} \equiv\left(d_{ \pm}^{\text {oct-intr }}\right)^{2}
$$

where

$$
\begin{aligned}
d_{ \pm}^{\text {oct-intr }} & =\cos \Delta_{31}\left\{1-\left(\frac{C^{(+)}}{C^{(-)}}\right)^{2}\right\} \\
& \times\left[\frac{1}{4} \cos \Delta_{31}\left(C^{(-)}\right)^{2}\left\{1-\left(\frac{C^{(+)}}{C^{(-)}}\right)^{2}\right\} \pm C^{(-)}\left(s_{1} \cos \delta_{1}+R s_{1} \sin \delta_{1}\right)\right] .
\end{aligned}
$$


The smooth limit to the same-octant intrinsic degeneracy solution can be achieved by taking the sign convention

$$
\begin{aligned}
& s_{\mathrm{V}}^{2}=\frac{1}{2\left(1+R_{2}^{2}\right)}\left[H_{ \pm}-d_{ \pm}^{\text {oct-intr }} \sqrt{\frac{D_{ \pm}^{\text {oct-intr }}}{\left(d_{ \pm}^{\text {oct-intr }}\right)^{2}}}\right], \\
& s_{\mathrm{VI}}^{2}=\frac{1}{2\left(1+R_{2}^{2}\right)}\left[H_{ \pm}+d_{ \pm}^{\text {oct-intr }} \sqrt{\frac{D_{ \pm}^{\text {oct-intr }}}{\left(d_{ \pm}^{\text {oct-intr }}\right)^{2}}}\right] .
\end{aligned}
$$

One can easily verify that in the maximum $\theta_{23}$ limit $s_{\mathrm{V}}$ and $s_{\mathrm{VI}}$ tend to $s_{1}$ and $s_{\mathrm{II}}$, respectively.

\section{B. Sign- $\Delta m_{31}^{2}$ degeneracy in the false $\theta_{23}$ octant}

We next discuss the sign- $\Delta m_{31}^{2}$ degeneracy in a $\theta_{23}$ octant different from the true one. The input solution $\left(s_{1}, \delta_{1}\right)$ and the false octant clone solution $\left(s_{7}, \delta_{7}\right)$ satisfy the following equations

$$
\begin{aligned}
& P=X_{ \pm}^{\text {true }} s_{1}^{2}+Y_{ \pm} s_{1}\left(\cos \delta_{1} \cos \Delta_{31} \mp \sin \delta_{1} \sin \Delta_{31}\right)+Z^{\text {true }} \\
& P=X_{\mp}^{\text {false }} s_{7}^{2}+Y_{\mp} s_{7}\left(\cos \delta_{7} \cos \Delta_{31} \pm \sin \delta_{7} \sin \Delta_{31}\right)+Z^{\text {false }}
\end{aligned}
$$

in the neutrino channel, and

$$
\begin{aligned}
& P^{C P}=X_{\mp}^{\text {true }} s_{1}^{2}-Y_{\mp} s_{1}\left(\cos \delta_{1} \cos \Delta_{31} \pm \sin \delta_{1} \sin \Delta_{31}\right)+Z^{\text {true }} \\
& P^{C P}=X_{ \pm}^{\text {false }} s_{7}^{2}-Y_{ \pm} s_{7}\left(\cos \delta_{7} \cos \Delta_{31} \mp \sin \delta_{7} \sin \Delta_{31}\right)+Z^{\text {false }}
\end{aligned}
$$

in the CP-conjugate channel. Proceeding along the same way as in Secs. IIIC and IV A we obtain

$$
\begin{aligned}
s_{7} \cos \delta_{7} & =\frac{1}{\cos \Delta_{31}} \frac{1}{\left(C_{2}^{(+)}\right)^{2}-\left(C_{2}^{(-)}\right)^{2}}\left[C_{2}^{(+)} T_{5 \pm}^{C P} \pm C_{2}^{(-)}\left(T_{6 \pm}^{C P}-2 s_{7}^{2}\right)\right], \\
s_{7} \sin \delta_{7} & =\frac{1}{\sin \Delta_{31}} \frac{1}{\left(C_{2}^{(+)}\right)^{2}-\left(C_{2}^{(-)}\right)^{2}}\left[C_{2}^{(-)} T_{5 \pm}^{C P} \pm C_{2}^{(+)}\left(T_{6 \pm}^{C P}-2 s_{7}^{2}\right)\right] .
\end{aligned}
$$

where we have defined

$$
\begin{aligned}
& T_{5 \pm}^{C P} \equiv \pm E_{2}^{(-)} s_{1}^{2} \mp G^{(-)}+D_{2}^{(+)} s_{1} \cos \delta_{1} \cos \Delta_{31}-D_{2}^{(-)} s_{1} \sin \delta_{1} \sin \Delta_{31}, \\
& T_{6 \pm}^{C P} \equiv E_{2}^{(+)} s_{1}^{2}+G^{(+)} \pm D_{2}^{(-)} s_{1} \cos \delta_{1} \cos \Delta_{31} \mp D_{2}^{(+)} s_{1} \sin \delta_{1} \sin \Delta_{31} .
\end{aligned}
$$

We have used the notations

$$
\begin{aligned}
D_{2}^{( \pm)} & \equiv \frac{Y_{+}}{X_{-}^{\text {false }}} \pm \frac{Y_{-}}{X_{+}^{\text {false }}}, \quad E_{2}^{( \pm)} \equiv \frac{X_{+}^{\text {true }}}{X_{-}^{\text {false }}} \pm \frac{X_{-}^{\text {true }}}{X_{+}^{\text {false }}} \\
G^{( \pm)} & \equiv\left(\frac{1}{X_{+}^{\text {false }}} \pm \frac{1}{X_{-}^{\text {false }}}\right)\left(Z^{\text {true }}-Z^{\text {false }}\right)
\end{aligned}
$$

Inserting (55) into $\cos ^{2} \delta_{7}+\sin ^{2} \delta_{7}=1$ we obtain the quartic equation of $s_{13}$,

$$
4\left(1+R_{2}^{2}\right) s_{7}^{4}-4 J_{ \pm} s_{7}^{2}+K_{ \pm}=0,
$$


which is actually a quadratic equation of $s_{13}^{2}$ because of the quadratic dependence on $s_{13}$ of $s_{7} \cos \delta_{7}$ and $s_{7} \sin \delta_{7}$. Thus, there exist only two physical (i.e., positive) solutions, which implies that the octant degeneracy is two-fold. In (58) $J$ and $K$ are defined as

$$
\begin{gathered}
J_{ \pm}=\frac{1}{4} \cos ^{2} \Delta_{31}\left(C_{2}^{(-)}\right)^{2}\left[1-\left(\frac{C_{2}^{(+)}}{C_{2}^{(-)}}\right)^{2}\right]^{2} \pm \frac{1}{\sin ^{2} \Delta_{31}}\left(\frac{C_{2}^{(+)}}{C_{2}^{(-)}}\right) T_{5 \pm}^{C P}+\left(1+R_{2}^{2}\right) T_{6 \pm}^{C P}, \\
K_{ \pm}=\left[\cot ^{2} \Delta_{31}+\left(\frac{C_{2}^{(+)}}{C_{2}^{(-)}}\right)^{2}\right]\left(T_{5 \pm}^{C P}\right)^{2} \pm \frac{2}{\sin ^{2} \Delta_{31}}\left(\frac{C_{2}^{(+)}}{C_{2}^{(-)}}\right) T_{5 \pm}^{C P} T_{6 \pm}^{C P}+\left(1+R_{2}^{2}\right)\left(T_{6 \pm}^{C P}\right)^{2} .
\end{gathered}
$$

Then, the sign- $\Delta m_{31}^{2}$ degeneracy solution across $\theta_{23}$ octant is given by

$$
s_{7}^{2}=\frac{1}{2\left(1+R_{2}^{2}\right)}\left[J_{ \pm}[ \pm]^{*} \sqrt{D_{ \pm}^{\text {oct-sign }}}\right]
$$

where $[ \pm]^{*}$ is the temporary sign to be specified below and $D_{ \pm}^{\text {oct-sign }}$ is defined as

$$
\begin{aligned}
D_{ \pm}^{\text {oct-sign }} & =\left\{1-\left(\frac{C_{2}^{(+)}}{C_{2}^{(-)}}\right)^{2}\right\}^{2}\left[\frac{1}{16} \cos ^{4} \Delta_{31}\left(C_{2}^{(-)}\right)^{4}\left\{1-\left(\frac{C_{2}^{(+)}}{C_{2}^{(-)}}\right)^{2}\right\}^{2}\right. \\
& \left.-\cot ^{2} \Delta_{31}\left(T_{5 \pm}^{C P}\right)^{2} \pm \frac{1}{2} \cot ^{2} \Delta_{31} C_{2}^{(+)} C_{2}^{(-)} T_{5 \pm}^{C P}+\frac{1}{2} \cos ^{2} \Delta_{31}\left(C_{2}^{(-)}\right)^{2}\left(1+R_{2}^{2}\right) T_{6 \pm}^{C P}\right] .
\end{aligned}
$$

The region defined by $D_{ \pm}^{\text {oct-sign }} \leq 0$ defines the region of no sign- $\Delta m_{31}^{2}$ degeneracy solutions in the false $\theta_{23}$ octant. The region of no sign-octant degeneracy solution is displayed as the white regions in the bottom two panels in Figs. 14 and 16 for superbeam type settings, SB1, MB1, and MB2. The corresponding informations for neutrino factory setting NF are given in Figs. 18 and 19. Once there is a solution, the same argument as before assures that the solutions for $s_{7}^{2}$ in (61) are positive definite.

To define unambiguously the sign- $\Delta m_{31}^{2}$ degeneracy solutions in the false $\theta_{23}$ octant we need the following new convention. That is, we need to take both the maximum $\theta_{23}$ and the vacuum limits.

Convention D: We take the convention such that $\left(s_{\mathrm{VII}}, \delta_{\mathrm{VII}}\right)$ and $\left(s_{\mathrm{VIII}}, \delta_{\mathrm{VIII}}\right)$ tend to $\left(s_{\mathrm{III}}^{\mathrm{vac}}, \delta_{\mathrm{III}}^{\mathrm{vac}}\right)$ and $\left(s_{\mathrm{IV}}^{\mathrm{vac}}, \delta_{\mathrm{IV}}^{\mathrm{vac}}\right)$, respectively, in the simultaneous maximum- $\theta_{23}$ and the vacuum limit.

One can easily show in the combined limit that $\lim _{\theta_{23} \rightarrow \pi / 4 \text {,vacuum }} D_{ \pm}^{\text {oct-sign }}=\left(d_{ \pm}^{\text {oct-sign }}\right)^{2}$, where $d_{ \pm}^{\text {oct-sign }}=d_{ \pm}^{\text {sign }}\left(\theta_{23}=\pi / 4\right)$ with $d_{ \pm}^{\text {sign }}$ being defined in 35 . The smooth limit to the maximum $\theta_{23}$ of the sign- $\Delta m_{31}^{2}$ degeneracy solutions can be achieved by taking the sign convention $\mathrm{D}$. The solutions read

$$
\begin{aligned}
& s_{\mathrm{VII}}^{2}=\frac{1}{2\left(1+R_{2}^{2}\right)}\left[J_{ \pm}-d_{ \pm}^{\text {oct-sign }} \sqrt{\frac{D_{ \pm}^{\text {oct-sign }}}{\left(d_{ \pm}^{\text {oct-sign }}\right)^{2}}}\right], \\
& s_{\mathrm{VIII}}^{2}=\frac{1}{2\left(1+R_{2}^{2}\right)}\left[J_{ \pm}+d_{ \pm}^{\text {oct-sign }} \sqrt{\frac{D_{ \pm}^{\text {oct-sign }}}{\left(d_{ \pm}^{\text {oct-sign }}\right)^{2}}}\right] .
\end{aligned}
$$




\section{STRUCTURE OF PARAMETER DEGENERACY}

We now make the structure of parameter degeneracy transparent based on knowledges obtained in the previous two sections by using CP-conjugate measurement. First, we summarize the relationships between the true and the degeneracy solutions.

\section{A. Mappings between the true and the degeneracy solutions}

Let us start by putting the relationship between each intrinsic degeneracy pair of the solutions in order. If we denote the relationship between the intrinsic degeneracy solution derived in Sec. III B as $s_{\mathrm{II}}=\zeta_{ \pm}\left(s_{1}, \delta_{1}, \theta_{23}^{\text {true }}\right)$ and $\delta_{\mathrm{II}}=\iota_{ \pm}\left(s_{1}, \delta_{1}, \theta_{23}^{\text {true }}\right)$ then the other intrinsic degeneracy pairs of the solutions satisfy

$$
\begin{array}{rlrl}
s_{\mathrm{IV}} & =\zeta_{\mp}\left(s_{\mathrm{III}}, \delta_{\mathrm{III}}, \theta_{23}^{\text {true }}\right), & \delta_{\mathrm{IV}}=\iota_{\mp}\left(s_{\mathrm{III}}, \delta_{\mathrm{III}}, \theta_{23}^{\text {true }}\right), \\
s_{\mathrm{VI}} & =\zeta_{ \pm}\left(s_{\mathrm{V}}, \delta_{\mathrm{V}}, \pi / 2-\theta_{23}^{\text {true }}\right), & & \delta_{\mathrm{VI}}=\iota_{ \pm}\left(s_{\mathrm{V}}, \delta_{\mathrm{V}}, \pi / 2-\theta_{23}^{\text {true }}\right), \\
s_{\mathrm{VIII}} & =\zeta_{\mp}\left(s_{\mathrm{VII}}, \delta_{\mathrm{VII}}, \pi / 2-\theta_{23}^{\text {true }}\right), & & \delta_{\mathrm{VIII}}=\iota_{\mp}\left(s_{\mathrm{VII}}, \delta_{\mathrm{VII}}, \pi / 2-\theta_{23}^{\text {true }}\right),
\end{array}
$$

completing the one-to-one correspondence between them. We then summarize the one-toone correspondence relations between the solutions which involve the $\Delta m_{31}^{2}$-sign and/or the $\theta_{23}$ octant flips:

$$
\begin{aligned}
s_{\mathrm{III}}=\xi_{ \pm}^{\mathrm{CP} \text { sign }}\left(s_{1}, \delta_{1}, \theta_{23}^{\text {true }}\right), & s_{\mathrm{IV}}=\xi_{ \pm}^{\mathrm{CP} \operatorname{sign}}\left(s_{\mathrm{II}}, \delta_{\mathrm{II}}, \theta_{23}^{\text {true }}\right), \\
s_{\mathrm{V}}=\xi_{ \pm}^{\mathrm{CP} \text { oct }}\left(s_{1}, \delta_{1}, \theta_{23}^{\text {true }}\right), & s_{\mathrm{VI}}=\xi_{ \pm}^{\mathrm{CP} \text { oct }}\left(s_{\mathrm{II}}, \delta_{\mathrm{II}}, \theta_{23}^{\text {true }}\right), \\
s_{\mathrm{VII}}=\xi_{ \pm}^{\mathrm{CP} \text { oct-sign }}\left(s_{1}, \delta_{1}, \theta_{23}^{\text {true }}\right), & s_{\mathrm{VIII}}=\xi_{ \pm}^{\mathrm{CP} \text { oct-sign }}\left(s_{\mathrm{II}}, \delta_{\mathrm{II}}, \theta_{23}^{\text {true }}\right), \\
s_{\mathrm{VII}}=\xi_{ \pm}^{\mathrm{CP} \text { sign }}\left(s_{\mathrm{V}}, \delta_{\mathrm{V}}, \pi / 2-\theta_{23}^{\text {true }}\right), & s_{\mathrm{VIII}}=\xi_{ \pm}^{\mathrm{CP} \text { sign }}\left(s_{\mathrm{VI}}, \delta_{\mathrm{VI}}, \pi / 2-\theta_{23}^{\text {true }}\right), \\
s_{\mathrm{VII}}=\xi_{\mp}^{\mathrm{CP} \text { oct }}\left(s_{\mathrm{III}}, \delta_{\mathrm{III}}, \theta_{23}^{\text {true }}\right), & s_{\mathrm{VIII}}=\xi_{\mp}^{\mathrm{CP} \text { oct }}\left(s_{\mathrm{IV}}, \delta_{\mathrm{IV}}, \theta_{23}^{\text {true }}\right), \\
s_{\mathrm{V}}=\xi_{\mp}^{\mathrm{CP} \text { oct-sign }}\left(s_{\mathrm{III}}, \delta_{\mathrm{III}}, \theta_{23}^{\text {true }}\right), & s_{\mathrm{VI}}=\xi_{\mp}^{\mathrm{CP} \text { oct-sign }}\left(s_{\mathrm{IV}}, \delta_{\mathrm{IV}}, \theta_{23}^{\text {true }}\right) .
\end{aligned}
$$

The functional form of $\xi_{ \pm}^{\mathrm{CP} \text { sign }}, \xi_{ \pm}^{\mathrm{CP} \text { oct}}$, and $\xi_{ \pm}^{\mathrm{CP} \text { oct-sign }}$ are defined in 37 in Sec. IIID, the first line in (52) in Sec. IV A, and the first line in (63), respectively. There exist the similar relationships between $\delta$ 's through the function $\eta_{ \pm}^{\mathrm{CP}}$ sign , but we do not display them explicitly here. The correspondence relations (65) between the true and the degeneracy solutions II-VIII are pictorially represented in Fig. 4. The relationship between the degeneracy solutions in (64) and (65) as well as in Fig. 4 is the precise meaning of the statement that nature of the parameter degeneracy is the intrinsic degeneracy duplicated respectively by the $\Delta m_{31}^{2}$-sign and the $\theta_{23}$ octant flips.

It is easy to prove 65 by considering the original defining equations for the degeneracy. For example, it is easy to show that $\xi_{ \pm}^{\mathrm{CP} \text { sign }}\left(s_{\mathrm{V}}, \delta_{\mathrm{V}}, \pi / 2-\theta_{23}^{\text {true }}\right) \equiv s_{\mathrm{X}}$ and $\eta_{ \pm}^{\mathrm{CP}}{ }^{\text {sign }}\left(s_{\mathrm{V}}, \delta_{\mathrm{V}}, \pi / 2-\right.$ $\left.\theta_{23}^{\text {true }}\right) \equiv \delta_{\mathrm{X}}$ are solutions of the equation

$$
\begin{aligned}
& X_{ \pm}^{(\text {false })} s_{\mathrm{V}}^{2}+Y_{ \pm} s_{\mathrm{V}} \cos \left(\delta_{\mathrm{V}} \pm \Delta_{31}\right)+Z^{(\text {false })}=X_{\mp}^{(\text {false })} s_{\mathrm{X}}^{2}+Y_{\mp} s_{\mathrm{X}} \cos \left(\delta_{\mathrm{X}} \mp \Delta_{31}\right)+Z^{(\text {false })} \\
& X_{\mp}^{(\text {false })} s_{\mathrm{V}}^{2}-Y_{\mp} s_{\mathrm{V}} \cos \left(\delta_{\mathrm{V}} \mp \Delta_{31}\right)+Z^{(\text {false })}=X_{ \pm}^{(\text {false })} s_{\mathrm{X}}^{2}-Y_{ \pm} s_{\mathrm{X}} \cos \left(\delta_{\mathrm{X}} \pm \Delta_{31}\right)+Z^{(\text {false })} .
\end{aligned}
$$

Note that left hand side of Eq.(66) can be expressed by $s_{1}, \delta_{1}$, and $\theta_{23}^{\text {true }}$ from Eq. (40) and (41), it is nothing but the equations of sign-octant degeneracy. 


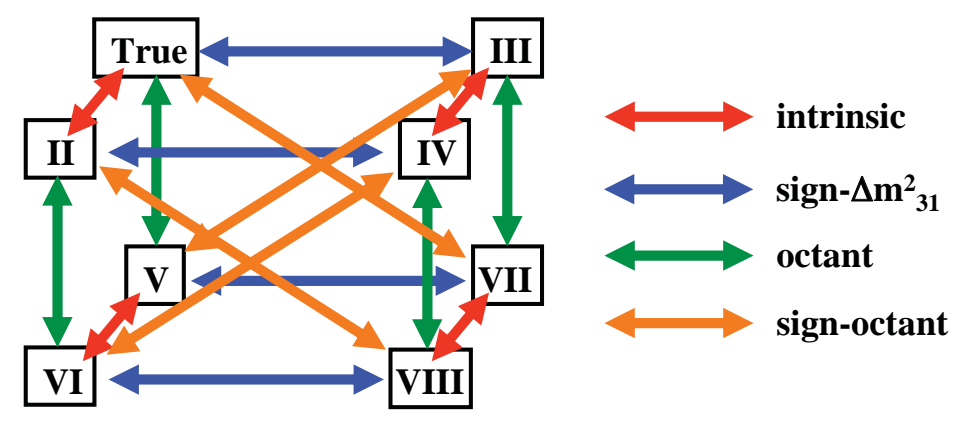

FIG. 4: The correspondence relations (65) between the solutions, the true one and the clones II-VIII, are pictorially represented.

Though we do not present explicit formulas the similar structure exists in all the degeneracy solutions in other settings, T-conjugate, Golden-Silver, and CPT-conjugate measurement to be discussed in the following sections.

\section{B. Relation between cases of true normal vs. true inverted mass hierarchies}

Here, we note an important property of the degeneracy solutions. Namely, if we know the degeneracy solutions for the input true normal mass hierarchy, then the solutions for the true inverted mass hierarchy can be immediately obtained from the former ones. ${ }^{6}$ Since the statement can be confusing to the readers, we give an explicit proof as below.

Let us take the sign- $\Delta m_{31}^{2}$ degeneracy (without $\theta_{23}$ octant flip) for definiteness. For clarity, we denote the degeneracy solution for the case of true normal and true inverted mass hierarchies as $\left(s_{3 \mathrm{~N}}, \delta_{3 \mathrm{~N}}\right)$ and $\left(s_{3 \mathrm{I}}, \delta_{3 \mathrm{I}}\right)$, respectively, where the subscript " 3 " implies either III or IV. For a given set of the probabilities $P$ and $P^{C P}$, assuming that the true mass hierarchy is normal, the true solution $\left(s_{1}, \delta_{1}\right)$ and the fake one $\left(s_{3 \mathrm{~N}}, \delta_{3 \mathrm{~N}}\right)$ satisfy

$$
\begin{aligned}
&(P=) X_{+} s_{1}^{2}+Y_{+} s_{1} \cos \left(\delta_{1}+\Delta_{31}\right)+Z=X_{-} s_{3 \mathrm{~N}}^{2}+Y_{-} s_{3 \mathrm{~N}} \cos \left(\delta_{3 \mathrm{~N}}-\Delta_{31}\right)+Z, \\
&\left(P^{C P}=\right) X_{-} s_{1}^{2}-Y_{-} s_{1} \cos \left(\delta_{1}-\Delta_{31}\right)+Z=X_{+} s_{3 \mathrm{~N}}^{2}-Y_{+} s_{3 \mathrm{~N}} \cos \left(\delta_{3 \mathrm{~N}}+\Delta_{31}\right)+Z .
\end{aligned}
$$

If the true mass hierarchy is inverted, then the degeneracy solution satisfies a different set of equations as

$$
\begin{aligned}
&(P=) X_{-} s_{1}^{2}+Y_{-} s_{1} \cos \left(\delta_{1}-\Delta_{31}\right)+Z=X_{+} s_{3 \mathrm{I}}^{2}+Y_{+} s_{3 \mathrm{I}} \cos \left(\delta_{3 \mathrm{I}}+\Delta_{31}\right)+Z \\
&\left(P^{C P}=\right) X_{+} s_{1}^{2}-Y_{+} s_{1} \cos \left(\delta_{1}+\Delta_{31}\right)+Z=X_{-} s_{3 \mathrm{I}}^{2}-Y_{-} s_{3 \mathrm{I}} \cos \left(\delta_{3 \mathrm{I}}-\Delta_{31}\right)+Z .
\end{aligned}
$$

We define $\delta_{1 \text { new }}$ and $\delta_{3 \text { Inew }}$ as $\delta_{1}=\delta_{1 \text { new }}+\pi$ and $\delta_{3 \mathrm{I}}=\delta_{3 \text { Inew }}+\pi$, respectively, and rewrite (68) by using them. It reads

$$
\begin{aligned}
& X_{-} s_{1}^{2}-Y_{-} s_{1} \cos \left(\delta_{1 \text { new }}-\Delta_{31}\right)+Z=X_{+} s_{3 \mathrm{I}}^{2}-Y_{+} s_{3 \mathrm{I}} \cos \left(\delta_{3 \text { Inew }}+\Delta_{31}\right)+Z, \\
& X_{+} s_{1}^{2}+Y_{+} s_{1} \cos \left(\delta_{1 \text { new }}+\Delta_{31}\right)+Z=X_{-} s_{3 \mathrm{I}}^{2}+Y_{-} s_{3 \mathrm{I}} \cos \left(\delta_{3 \text { Inew }}-\Delta_{31}\right)+Z .
\end{aligned}
$$

\footnotetext{
6 In spite of this charming and useful property we have been denoting explicitly the input mass hierarchies as the \pm signs in the subscript for clarity and simplicity of keep tracking of the true mass hierarchy we are working.
} 
Comparison between (67) and (69) tells us that if the set $\left(s_{3 \mathrm{~N}}, \delta_{3 \mathrm{~N}}\right)$ is the solution to the sign- $\Delta m_{31}^{2}$ degeneracy equation for the true normal hierarchy, then the set $\left(s_{3 \mathrm{I}}, \delta_{3 \mathrm{Inew}}\right)$ is the solution for the true inverted hierarchy, provided that the true value of $\delta\left(=\delta_{1}\right)$ is replaced by $\delta_{1 \text { new }}$. Stated more explicitly, if the first two equations in 12 define the sign- $\Delta m_{31}^{2}$ degeneracy solution for the true normal hierarchy,

$$
s_{3 \mathrm{~N}}=\xi_{3}\left(s_{1}, \delta_{1}\right), \quad \delta_{3 \mathrm{~N}}=\eta_{3}\left(s_{1}, \delta_{1}\right)
$$

then, the degeneracy solution for the true inverted hierarchy is given, if expressed in terms of the true input parameters, as

$$
s_{3 \mathrm{I}}=\xi_{3}\left(s_{1}, \delta_{1}-\pi\right), \quad \delta_{3 \mathrm{I}}=\eta_{3}\left(s_{1}, \delta_{1}-\pi\right)+\pi .
$$

In other word, the mapping functions $\xi_{ \pm}$and $\eta_{ \pm}$are related with each other as

$$
\begin{aligned}
& \xi_{-}^{\mathrm{CP} \operatorname{sign}}\left(s_{1}, \delta_{1}\right)=\xi_{+}^{\mathrm{CP} \operatorname{sign}}\left(s_{1}, \delta_{1}-\pi\right), \\
& \eta_{-}^{\mathrm{CP} \operatorname{sign}}\left(s_{1}, \delta_{1}\right)=\eta_{+}^{\mathrm{CP} \operatorname{sign}}\left(s_{1}, \delta_{1}-\pi\right)+\pi .
\end{aligned}
$$

Thus, the sign- $\Delta m_{31}^{2}$ degeneracy solutions for the true inverted hierarchy are essentially determined by the solutions for the true normal hierarchy. The relationship between the degeneracy solutions with the normal and the inverted hierarchies as the true solution may be understood better by comparing the no-slotuion regions given in Fig. 3 which are drawn with the inverted hierarchy as the input true solution to those of Figs. 10 and 12 .

It can be easily seen that the same treatment goes through for other types of degeneracies, the intrinsic and the sign- $\Delta m_{31}^{2}$ degeneracies in the same or the different $\theta_{23}$ octants. Therefore, the relationship (72) holds for the whole eightfold degeneracy.

\section{Asymptotic expansion}

At the end of this section, which is devoted to illuminate general properties of the degeneracy solutions, we make comments on high-energy behavior of the solutions. It would help clarifying some features of the energy dependence of the degeneracy solutions which are discussed in the next section.

First of all, it can be easily verified that all the degeneracy solutions $s_{\mathrm{N}}$ and $\delta_{\mathrm{N}}$ $(\mathrm{N}=\mathrm{II}-\mathrm{VIII})$ derived in Secs. III and IV have finite asymptotic limit as $E \rightarrow \infty$. Then, we note an interesting property that they are invariant under the transformation $E \rightarrow-E$, or $\Delta_{j 1} \rightarrow-\Delta_{j 1}(j=2,3)$. The invariance, of course, stems from the one of the oscillation probabilities. Though the transformation is unphysical in nature, the transformation property is useful. It means that when we do asymptotic expansion of the degeneracy solutions as $s_{\mathrm{N}}=\sum_{n=0} a_{n}^{\mathrm{N}}\left(\Delta_{31}\right)^{n}$ the odd terms are absent:

$$
\begin{aligned}
& s_{\mathrm{N}}=a_{0}^{\mathrm{N}}+a_{2}^{\mathrm{N}} \Delta_{31}^{2}+\mathcal{O}\left(\Delta_{31}^{4}\right), \\
& \delta_{\mathrm{N}}=b_{0}^{\mathrm{N}}+b_{2}^{\mathrm{N}} \Delta_{31}^{2}+\mathcal{O}\left(\Delta_{31}^{4}\right) .
\end{aligned}
$$

Absence of the first-order term in $1 / E$ implies that onset to the high-energy asymptotic behavior of the degeneracy solutions is relatively fast, as we will confirm in the next section. 


\section{OVERVIEW OF THE EIGHTFOLD PARAMETER DEGENERACY IN CP- CONJUGATE MEASUREMENT}

In this section, we try to give an overview of the intrinsic, the sign- $\Delta m_{31}^{2}$, and the $\theta_{23}$ octant degeneracies. In fact, the features of the degeneracy solutions are quite different for differing baselines and neutrino energies, which makes the overview in a genuine sense extremely difficult. Therefore, we restrict ourselves in this paper into a few typical settings which may be relevant for the settings of future neutrino experiments discussed in the literatures. If the readers want to examine features of the degeneracy with some alternative experimental parameters, they can do it quite easily by using the analytic solutions presented in this paper.

We want to warn the readers that all of our comments to be made in this section are qualitative in nature. Therefore, when we say, "spectrum analysis would resolve the degeneracy" it actually means that it may be possible to resolve it if appropriate experimental settings are provided. ${ }^{7}$ Yet, we try to be based the experiences gained in some previous analyses. The readers may still wonder whether the discussion of degeneracy based on the probability makes sense because the observable in the experiments must be obtained after convolution with neutrino fluxes and cross sections. However, this is not the only possible attitude to take. One can, in principe, obtain the "experimental data of probability" by de-convoluting the fluxes and cross sections as shown in [80].

We also remind the readers of the fact that limited statistics in measurement has a nontrivial impact on the features of the degeneracy to be observed in the actual experiments. If two (or more) degeneracy solutions exist in nearby locations in the mixing parameter space they can merge together, producing an apparent single solution, which could be misinterpreted as no degeneracy. We have discussed in Secs. III and IV] the regions of no degenerate solutions. However, there might be cases that the degeneracy solutions do exist in these regions because of lack of statistics and/or shift of the allowed regions due to systematic uncertainties. Discussion of these features is outside the scope of this paper.

\section{A. Variables used for display and baselines and neutrino energies adopted}

We try to illuminate some characteristic features of the degeneracy by presenting the differences between the true solution and the fake ones. To display the difference between the solutions we define the ratio $R_{\mathrm{N}}$ as

$$
R_{\mathrm{N}} \equiv \frac{\sin ^{2} 2 \theta_{13}^{\mathrm{N}}-\sin ^{2} 2 \theta_{13}^{\text {true }}}{\sin ^{2} 2 \theta_{13}^{\text {true }}}
$$

where $\mathrm{N}=$ II-VIII denote the degeneracy solution labels. We use the variable $\sin ^{2} 2 \theta_{13}$ because it is closer to the experimentally measured quantity. Similarly, we define the quantity $D_{\mathrm{N}}$ to represent the differences between the true and the clone solutions. For this purpose,

\footnotetext{
7 Similarly, when we say "the degeneracy A is easier to lift than the degeneracy B" it actually means so provided that an appropriate experimental condition is prepared such that the similar sensitivities would be expected for both the solutions A and B.
} 
there are two appropriate ways to define it, the types (1) and (2);

$$
D_{\mathrm{N}}^{(1)} \equiv \frac{\left(\delta^{\mathrm{N}}-\delta^{\text {true }}\right)}{\pi}, \quad D_{\mathrm{N}}^{(2)} \equiv \frac{\delta^{\mathrm{N}}-\left(\pi-\delta^{\text {true }}\right)}{\pi} .
$$

We use either one of $D_{\mathrm{N}}^{(1)}$ or $D_{\mathrm{N}}^{(2)}$ whichever appropriate depending upon the degeneracy types.

As typical experimental settings, we use the following four cases of baselines and neutrino energies:

- SB1: $L=300 \mathrm{~km}, E=700 \mathrm{MeV}$; A short baseline low energy $\nu_{\mu}$ (and $\bar{\nu}_{\mu}$ ) superbeam near the first oscillation maximum

- MB1: $L=1000 \mathrm{~km}, E=2.33 \mathrm{GeV}$; A medium baseline superbeam near the first oscillation maximum

- MB2: $L=1000 \mathrm{~km}, E=780 \mathrm{MeV}$; A medium baseline superbeam near the second oscillation maximum

- NF: $L=4000 \mathrm{~km}, E=20 \mathrm{GeV}$; A typical setting for neutrino factory

The first three settings, SB1, MB2, and MB1, are examined in the following three subsections (Secs. VIB, VIC, and VID), while the last one, NF, in Sec. VIE, We should remark here that the energies corresponding to each baseline are chosen rather arbitrarily just for display, while their order of magnitudes are dictated by the baseline distances. ${ }^{8}$ In some limited cases, the features of degeneracy solutions of the first two cases, SB1 and MB1, are so similar that we omit MB1 plots. Comparison between MB1 and MB2 settings, former (latter) being around the first (second) oscillation maximum, would be interesting to know physics behind the potential of the two-detector setting [81] and/or the BNL-type wide band beam approach 52 .

The key to resolve the degeneracy is to utilize spectrum informations. Therefore, we also present the energy dependence of the difference between the true and the degeneracy solutions. A point of interest is how the energy dependence differs among the three different types of the degeneracies.

We note that in all the figures presented in this section we take the normal mass hierarchy as the input true solution. If one wants to have the corresponding informations for the inverted mass hierarchy, one can do it just by changing the ordinate label of the figures as $\delta^{\text {true }}-\pi$ not $\delta^{\text {true }}$. This is discussed in detail in Sec. VB.

We use the following values for the mixing parameters as summarized below: $\Delta m_{21}^{2}=$ $7.9 \times 10^{-5} \mathrm{eV}^{2}, \sin ^{2} \theta_{12}=0.31$, and $\Delta m_{31}^{2}=2.5 \times 10^{-3} \mathrm{eV}^{2}$. The matter density is taken as $\rho=2.8 \mathrm{~g} / \mathrm{cm}^{3}$ for SB1, MB1, and MB2 settings, and $\rho=3.6 \mathrm{~g} / \mathrm{cm}^{3}$ for NF setting.

8 To avoid the energy of perfect oscillation maximum of about $600 \mathrm{MeV}$ at $300 \mathrm{~km}$, where the features of the degeneracy are special, we tentatively added $100 \mathrm{MeV}$. Hence, $\epsilon$, the deviation of $\Delta_{31}$ from $\pi / 2$ is given by $\Delta_{31}(L=300 \mathrm{~km}, E=700 \mathrm{MeV})=\pi / 2-\epsilon$ from which $\epsilon$ is determined as $\epsilon=\frac{\pi}{2} \frac{1}{7}=0.224$. For the second oscillation maximum, we have arbitrarily chosen that $\Delta_{31}(L=1000 \mathrm{~km}, E)=3(\pi / 2-\epsilon)$, that is $E=780 \mathrm{MeV}$. 


\section{B. Intrinsic degeneracy in the true $\theta_{23}$ octant}

In Figs. 5 and 6 , we present $R_{\mathrm{II}}$, the normalized difference of $\sin ^{2} 2 \theta_{13}$, and $D_{\mathrm{II}}^{(2)}$, a difference of $\delta / \pi$ defined in (75), respectively, between the true and the intrinsic degeneracy solutions for the two typical cases of energies and baselines, SB1 and MB2. We do not present the same plots for MB1 setting because they are so similar to those of SB1. A color variation is used to clearly represent the ratio $R_{\mathrm{II}}$ and $D_{\mathrm{II}}^{(2)}$ in a visual way, which will be used later also to all of $R_{\mathrm{N}}$ and $D_{\mathrm{N}}^{(i)}$. From blue to red $R_{\mathrm{II}}$ and $D_{\mathrm{II}}^{(2)}$ vary from -1 to +1 . The only exception to this rule is $R_{\mathrm{N}}$ at color graduation of the deepest red; It contains the region with $R_{\mathrm{N}}$ greater than 1 . Notice that there is no region of $R_{\mathrm{N}}<-1$ by definition in (74). ${ }^{9}$ We should note that in the case of $D_{\mathrm{N}}^{(i)}$, unlike $R_{\mathrm{N}}$, the deep blue region smoothly continues to the deep red because of the periodicity in $\delta$.
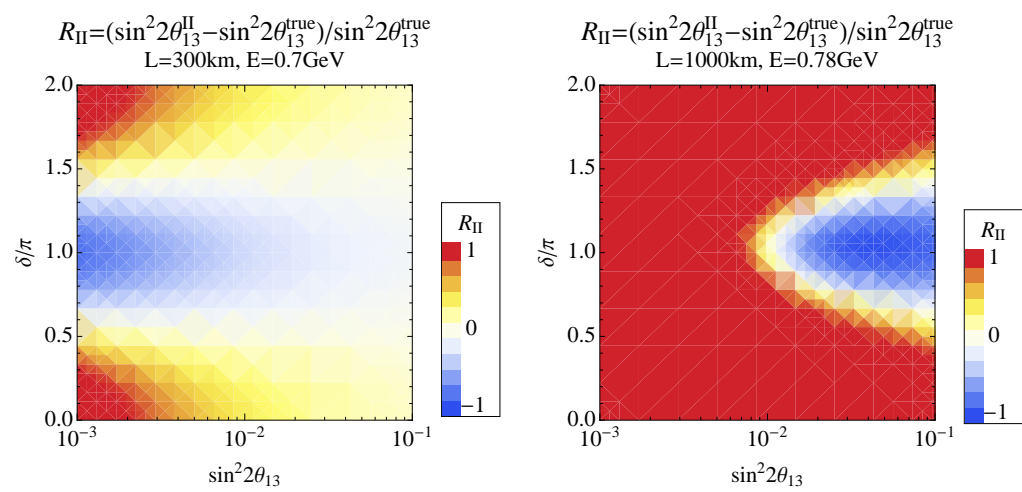

FIG. 5: The ratio $R_{\mathrm{II}}=\left[\sin ^{2} 2 \theta_{13}^{\mathrm{N}}-\sin ^{2} 2 \theta_{13}^{\text {true }}\right] / \sin ^{2} 2 \theta_{13}^{\text {true }}$ defined in $\left(74\right.$ ) is presented in $\sin ^{2} 2 \theta_{13}^{\text {true }}-$ $\delta^{\text {true }} / \pi$ space for the two typical cases of energies and baselines, SB1 and MB2, defined in Sec. VIA. We do not present the same plots for the setting MB1 because they are so similar to those of SB1.
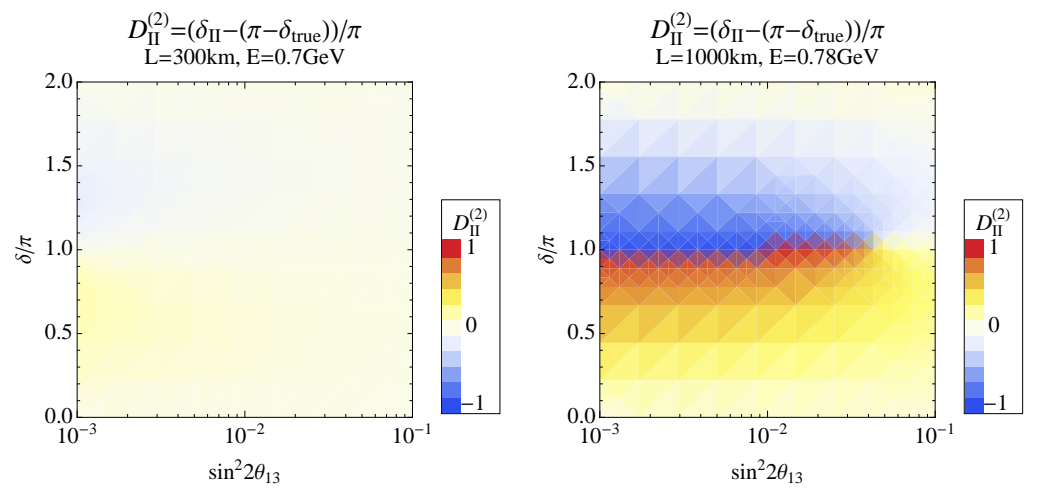

FIG. 6: The normalized difference $D_{\mathrm{II}}^{(2)} \equiv\left[\delta^{\mathrm{II}}-\left(\pi-\delta^{\text {true }}\right)\right] / \pi$, defined in 75$)$ is presented in $\sin ^{2} 2 \theta_{13}^{\text {true }}-\delta^{\text {true }} / \pi$ space for the two typical settings SB1 and MB2 defined in Sec. VIA. We do not present the same plots for the setting MB1 because they are so similar to those of SB1.

9 Here we need to mention about some details of color variation. 20 color graduation are used to draw $R_{\mathrm{N}}$ and $D_{\mathrm{N}}^{(i)}$ so that a single color graduation spans $5 \%$ of the entire region. 

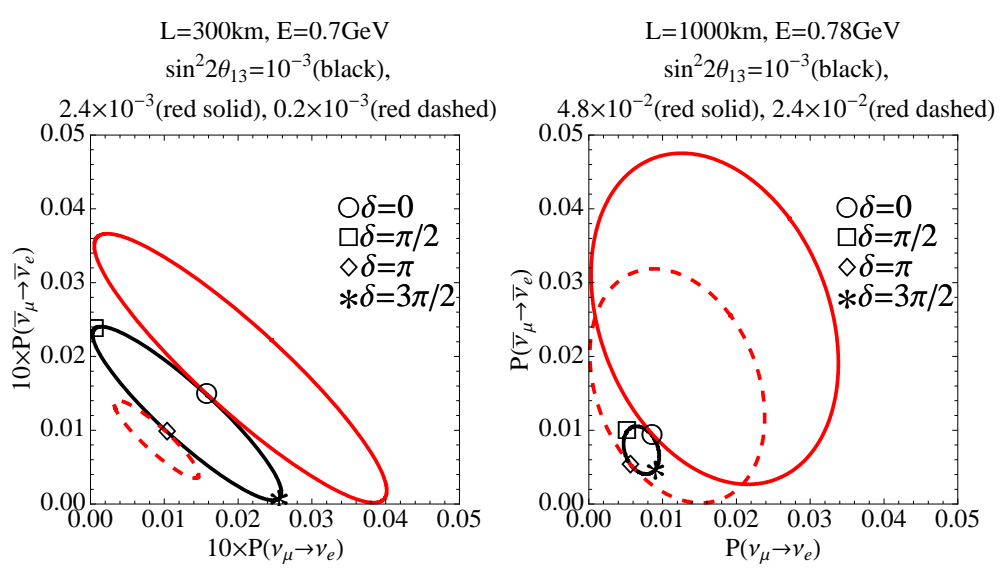

FIG. 7: Bi-probability plot for settings of SB1 (left panel) and MB2 (right panel). In both plots the true value of $\theta_{13}$ is taken to be $\sin ^{2} 2 \theta_{13}=10^{-3}$. The ellipses of the intrinsic degeneracy solutions are depicted by the red solid and red dashed lines.

One of the most notable features in Fig. 5 is a clear difference between SB1 (left panel) and MB2 (right panel) settings. In large $\theta_{13}$ region in SB1 setting, $\sin ^{2} 2 \theta_{13} \gtrsim 10^{-2}, R_{\mathrm{II}}$ is small. At small $\theta_{13}$ in SB1 setting and at large $\theta_{13}$ in MB2 setting, $R_{\mathrm{II}}$ is large and positive (negative) at $\delta \sim 0(\pi)$. In small $\theta_{13}$ region in MB2 setting, $\sin ^{2} 2 \theta_{13} \lesssim 10^{-2}, R_{\mathrm{II}}$ is large and positive independent of $\delta .{ }^{10}$ Let us understand these features.

We start from the above first feature. It can be understood by the analytic solution (18). If $\theta_{13}$ is relatively large, $s_{13} \gg \Delta m_{21}^{2} / \Delta m_{31}^{2}, s_{1}$ is the dominant term in $s_{\text {II }}$ in (21), and $R_{\mathrm{II}} \sim \mathcal{O}\left(s_{1}^{-1} \Delta m_{21}^{2} / \Delta m_{31}^{2}\right)$. Therefore, $R_{\mathrm{II}}$ is small at large $\theta_{13}$ in SB1 setting. Now, the behavior of $R_{\mathrm{II}}$ at small $\theta_{13}$ can be easily understood by looking into the bi-probability plot, the left panel in Fig. 7. The degeneracy ellipse which shares the point around $\delta=0(\delta=\pi)$ of the true ellipse is the dashed (solid) one with considerably larger (smaller) $\theta_{13}$. The similar consideration explains the feature of $R_{\mathrm{II}}$ at large $\theta_{13}$ in MB2 setting. The remaining feature that needs explanation is the large positive $R_{\mathrm{II}}$ at small $\theta_{13}$ in MB2 setting. At such small $\theta_{13}$ as $\sin ^{2} 2 \theta_{13} \sim 10^{-3}$ and the baseline $L=1000 \mathrm{~km}$, the oscillation probability is dominated by the solar term $Z$. Since it is independent of $\delta$ the probability ellipse shrinks to a small "circle", as can be seen in the right panel in Fig. 7. Then, the degeneracy solution ellipses are inevitably large as indicated by the red solid and dashed lines, resulting degenerate solutions much larger than the true $s_{13}$.

We observe for $D_{\mathrm{II}}^{(2)}$ plotted in Fig. 6 that in SB1 (and MB1) setting $D_{\mathrm{II}}^{(2)}$ is small in the entire region of $\sin ^{2} 2 \theta_{13}-\delta / \pi$ space covered. ${ }^{11}$ It means that the approximate formula $\delta^{\mathrm{II}} \simeq \pi-\delta^{\text {true }}$ works well [23]. It is a nice feature of measurement by SB1 setting because $\mathrm{CP}$ violation is unlikely to be confused with $\mathrm{CP}$ conservation. Whereas for MB2 setting $D_{\text {II }}^{(2)}$ is small only in a limited region $-0.2 \lesssim \delta / \pi \lesssim 0.2$, and in a small strip around $\delta \simeq 0$ at large $\theta_{13}$. The deviation from the approximation $\delta^{\mathrm{II}} \simeq \pi-\delta^{\text {true }}$ is significant in the

10 At extremely small $\theta_{13}$, the left plot in Fig. 5 for SB1 almost looks like the right plot for MB2, but with scale of $\sin ^{2} 2 \theta_{13}$ two orders of magnitude smaller than that of the right panel in Fig. 5 .

11 Hereafter, when we talk about $\sin ^{2} 2 \theta_{13}$ and $\delta$ such as $\sin ^{2} 2 \theta_{13}-\delta / \pi$ space, it actually means the $\sin ^{2} 2 \theta_{13}^{\text {true }}$ and $\delta^{\text {true }}$, respectively. We use the simpler notation to avoid cumbersome superscript "true" as much as possible. 
second and the third quadrants of $\delta$, in particular in region $\sin ^{2} 2 \theta_{13} \lesssim$ a few $\times 10^{-2}$ in MB2 setting. It is possible to understand this behavior of $D_{\mathrm{II}}^{(2)}$ qualitatively at very small $\theta_{13}$, $\sin ^{2} 2 \theta_{13} \sim 10^{-3}$ by using the bi-probability plot for MB2 setting (right panel in Fig. 7). For the true value of $\delta^{\text {true }} \simeq 0$ the degeneracy ellipse is depicted by the solid line and $\delta_{\mathrm{II}} \simeq \pi$, which implies $D_{\mathrm{II}}^{(2)} \ll 1$. On the other hand, for $\delta^{\text {true }} \simeq \pi$ the degeneracy ellipse depicted by the dashed line touches to the true ellipse also at around $\delta \simeq \pi$, hence $D_{\mathrm{II}}^{(2)}$ is of order unity. Notice again that the deep blue region smoothly continues to the deep red because of the periodicity in $\delta$.
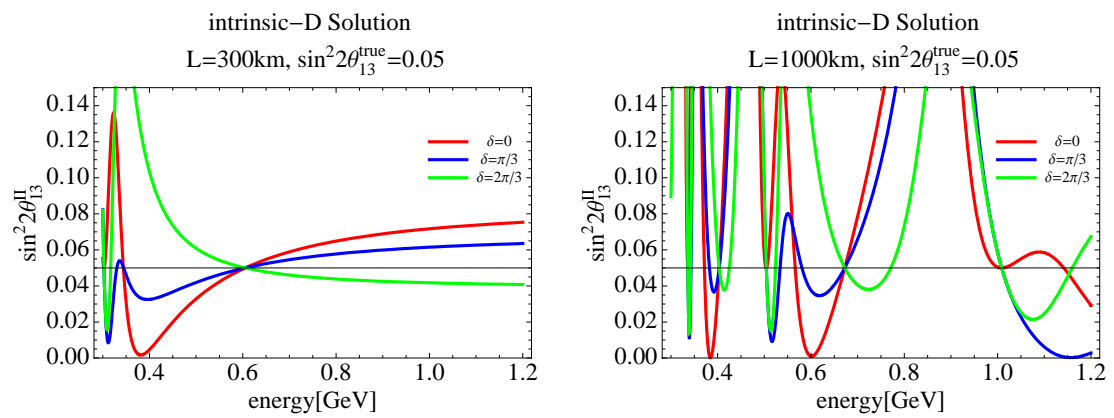

FIG. 8: The energy dependence of $\sin ^{2} 2 \theta_{13}^{\mathrm{II}}$ is plotted for the two typical settings SB1 (left panel) and MB2 (right panel) defined in Sec. VIA. The true value of $\theta_{13}$ is taken as $\sin ^{2} 2 \theta_{13}=0.05$, which is indicated by the horizontal solid line in the figure.
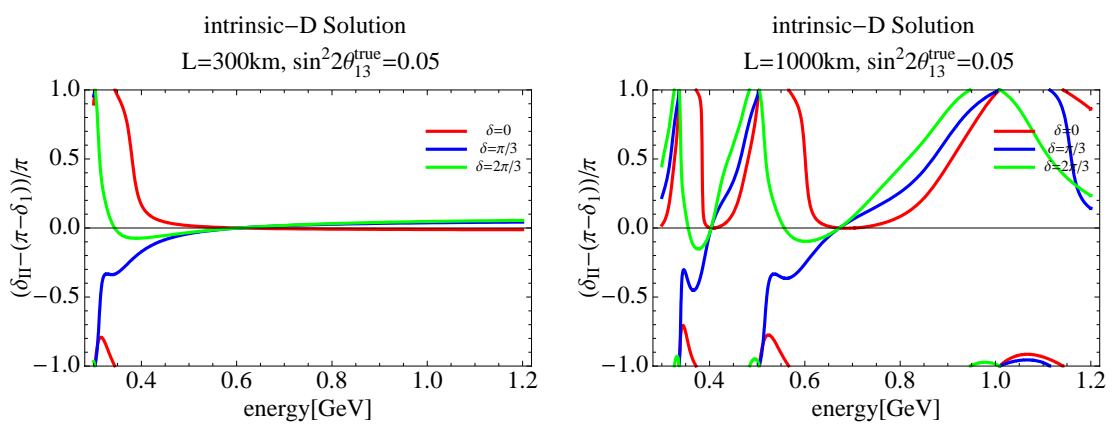

FIG. 9: The energy dependence of the ratio $D_{\mathrm{II}}^{(2)}$ defined in 75 is plotted for the two typical settings SB1 (left panel) and MB2 (right panel) defined in Sec. VI A. The true value of $\theta_{13}$ is taken as $\sin ^{2} 2 \theta_{13}=0.05$.

One of the most important issues to find possible ways to resolve the degeneracy is to know the energy dependence of the difference between the true and the clone solutions. Therefore, we present in Fig. 8 the energy dependence of $\sin ^{2} 2 \theta_{13}^{\mathrm{II}}$ for SB1 (left panel) and MB2 (right panel) settings, assuming the true value of $\sin ^{2} 2 \theta_{13}=0.05$. It may be regarded as a typical value for relatively large $\theta_{13}$ to which we will have an access by the ongoing experiments. Hereafter, whenever we present the similar figures of the energy dependence of the degeneracy solutions, we use only the three values of $\delta, \delta=0, \pi / 3$, and $2 \pi / 3$, for better visibility. (We have tried $\delta=0,2 \pi / 3$, and $4 \pi / 3$, but the latter two curves tend to overlap.) Similarly, in Fig. 9 the energy dependence of $D_{\mathrm{II}}^{(2)}$ is plotted for the same settings, 
SB1 and MB2, with the same true value of $\theta_{13}$. Again, we do not present the case of MB1 setting because the plots are very similar to those of SB1 apart from minor differences at low energies, $E \lesssim 1 \mathrm{GeV} .{ }^{12}$

Clearly, there exists a significant energy dependence of $\sin ^{2} 2 \theta_{13}^{\mathrm{II}}$ even for SB1 setting. One can see that they vary by a factor of $2-4(30 \%-40 \%)$, or more at low (high) energies depending upon $\delta$ in region of $E=0.4-1.2 \mathrm{GeV}$ for $L=300 \mathrm{~km}$. It must be contrasted to almost flat curves of energy dependence given in the following subsections, Figs. 11 (Sec. VIC) and 15 (Sec. VID) for the sign- $\Delta m_{31}^{2}$ and the octant degeneracies, respectively. Then, the spectrum analysis must be powerful in resolving the intrinsic degeneracy. It has been seen to be the case in the analysis of T2K II experiment [13] done in [53]. For $D_{\mathrm{II}}^{(2)}$ the energy dependence is significant only at low energies, below the first oscillation maximum, where usually the signal-to-background ratio is not helpful. Therefore, potential power for the spectrum analysis relies more on the energy dependence of $\sin ^{2} 2 \theta_{13}^{\mathrm{II}}$ not on $\delta_{\mathrm{II}}$ 's.

The energy dependences of $\sin ^{2} 2 \theta_{13}^{\mathrm{II}}$ and $D_{\mathrm{II}}^{(2)}$ plotted for MB2 setting magnify the low energy part of MB1 setting (not shown) at the same baseline of $L=1000 \mathrm{~km}$. The energy dependences are far more pronounced and depend sensitivity on $\delta$, and at some particular energies $\sin ^{2} 2 \theta_{13}^{\mathrm{II}}$ and $D_{\mathrm{II}}^{(2)}$ are pinned to $\sin ^{2} 2 \theta_{13}^{\text {true }}$ and zero, respectively. To understand better these features, we first note that the oscillation probabilities show violent energy dependences below the first oscillation maximum. The $\delta$-dependent strong energy dependence of the degeneracy solutions, however, is under restriction by pinning to the true value or zero at the energies corresponding to the $n$-th oscillation maxima $(n=1,2,3)$, as seen in the left and the right panels of Fig. 8. In SB1 setting $(300 \mathrm{~km})$ the first oscillation maximum is reached at $E \simeq 600 \mathrm{MeV}$, while for $L=1000 \mathrm{~km}$ the first, second and the third minima are approximately at $E \simeq 2 \mathrm{GeV}, 700 \mathrm{MeV}$, and $400 \mathrm{MeV}$, respectively. The zeros of $\sin ^{2} 2 \theta_{13}^{\mathrm{II}}-\sin ^{2} 2 \theta_{13}^{\text {true }}$ and $D_{\mathrm{II}}^{(2)}$ arise due to the special feature of the parameter degeneracy at the $n$-th oscillation maxima [27, 28], which can be seen explicitly from the formulas in Sec. IIIB, It can also be intuitively understood by having a flattened ellipse in the biprobaility diagram at the oscillation maxima $[24] .{ }^{13}$ The regularity of alternating "pinning to zero" and violent energy dependence gives us at least clear picture of what is seen in energy dependence of the degeneracy solutions in MB2 setting. The similar features will be seen in many figures of energy dependence in MB2 setting presented in the rest of this paper, which will allow analogous explanations.

Despite a possibility of confusion due to too complicated dependence on energy and $\delta$ at MB2 only setting, combination of MB2 at somewhat off the oscillation maxima with more quiet SB1 settings would be an ideal machinery for resolving the degeneracy. This was observed to occur in [53, 54] which utilizes the informations at the second oscillation maximum by a Korean detector, and most probably gives an explanation for high sensitivity achievable in the BNL-type wide band beam strategy [52, 57].

12 For more extensive presentation of these plots including those of MB1 setting and energy dependence plots with other values of $\delta$, see [79], and partly [82].

13 The similar zero at $E=1 \mathrm{GeV}$ can be understood as shrinking the ellipse into a small size at the oscillation minimum and is not interesting to us. 


\section{Sign- $\Delta m_{31}^{2}$ degeneracy in the true $\theta_{23}$ octant}

We now turn to the sign- $\Delta m_{31}^{2}$ degeneracy which exists in the same $\theta_{23}$ octant as the true one. Since there are two solutions, $\left(s_{\mathrm{III}}, \delta_{\mathrm{III}}\right)$ and $\left(s_{\mathrm{IV}}, \delta_{\mathrm{IV}}\right)$, we present them in the same figures. In Fig. $10, R_{\mathrm{III}}$ and $R_{\mathrm{IV}}$ defined in $(74)$ are plotted in $\sin ^{2} 2 \theta_{13}-\delta / \pi$ space. White region is the region of no degenerate solution as discussed in Sec. IIIC. We now show also MB1 case because difference from SB1 setting becomes non-negligible, in particular in small $\theta_{13}$ region. Nevertheless, the difference is not so significant at large $\theta_{13} \sin ^{2} 2 \theta_{13} \gtrsim 10^{-2}$ apart from the change in the no-solution region.
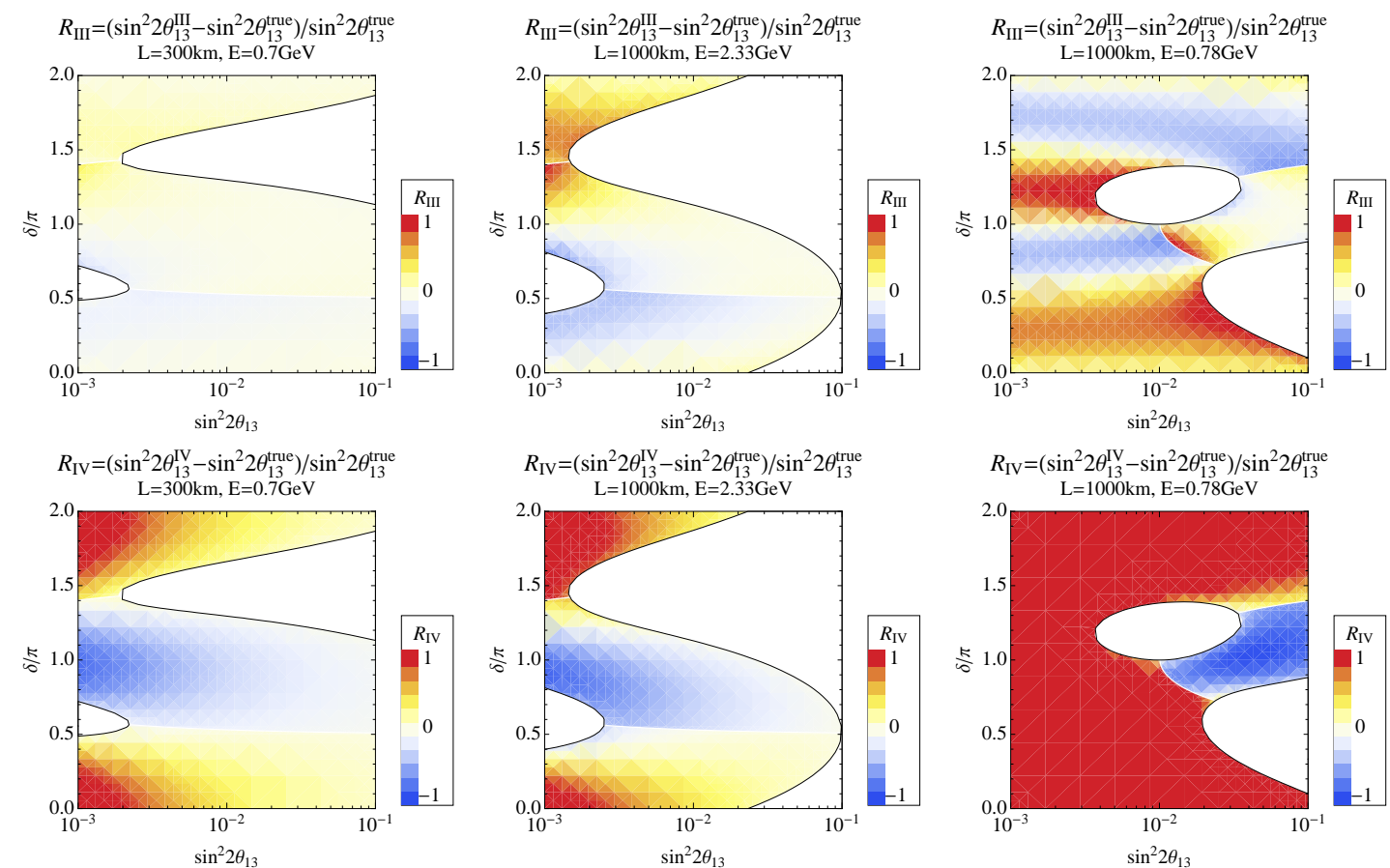

FIG. 10: The ratios $R_{\mathrm{III}}=\left[\sin ^{2} 2 \theta_{13}^{\mathrm{III}}-\sin ^{2} 2 \theta_{13}^{\text {true }}\right] / \sin ^{2} 2 \theta_{13}^{\text {true }}$ (upper three panels) and $R_{\mathrm{IV}}$ (lower three panels) defined in 74 in $\sin ^{2} 2 \theta_{13}-\delta / \pi$ space is presented for the three typical settings SB1 (left panel), MB1 (middle panel), and MB2 (right panel) defined in Sec. VIA. The regions of white color denote the regions of no sign-degeneracy solution.

By comparing Fig. 10 with Fig. 5 , it is evident that the difference of $\sin ^{2} 2 \theta_{13}$ between the true solution $s_{1}$ and $s_{\mathrm{III}}$ is much smaller than the case of intrinsic degeneracy solutions for SB1 and MB1 settings. Furthermore, the energy dependence of $\sin ^{2} 2 \theta_{13}^{\mathrm{III}}$ is much milder than the case of solution $\left(s_{\mathrm{II}}, \delta_{\mathrm{II}}\right)$ of the intrinsic degeneracy, as one can clearly see by comparing Fig. 11 with Fig. 8. These features make resolution of the sign- $\Delta m_{31}^{2}$ degeneracy much more difficult compared to the intrinsic degeneracy in these settings.

The difference between energy dependences of the intrinsic and the sign- $\Delta m_{31}^{2}$ degeneracies in SB1 and MB1 settings can be easily understood at least qualitatively. As we learned in Sec. VC, by lacking $\sim 1 / E$ terms, reach to high-energy asymptotic behavior is relatively fast. At low energies, $\sin ^{2} 2 \theta_{13}^{\mathrm{III}}$ is constrained to be small as one can show by the formulas based on the matter perturbation theory given in Appendix A. The first order correction term, from which the energy dependence comes in is small, of the order of 

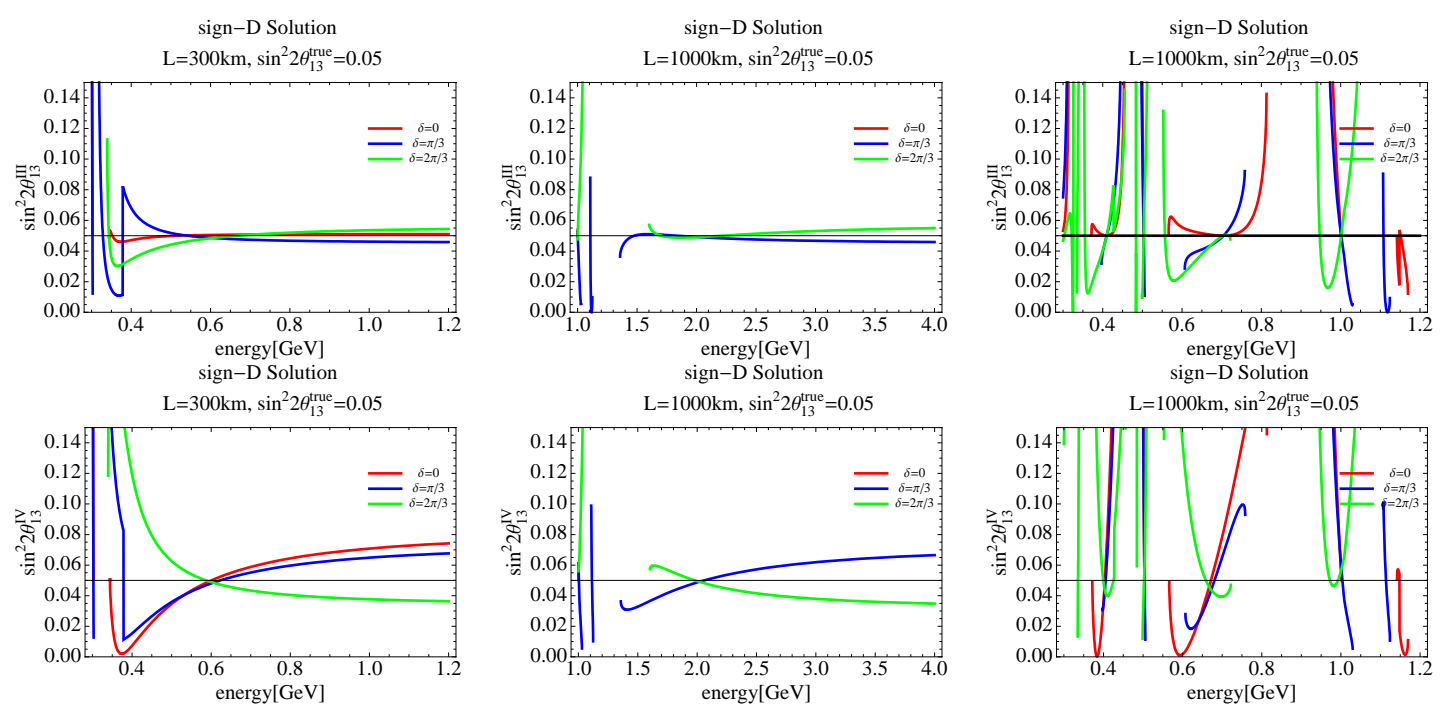

FIG. 11: The energy dependence of $\sin ^{2} 2 \theta_{13}^{\mathrm{III}}$ (upper three panels) and $\sin ^{2} 2 \theta_{13}^{\mathrm{IV}}$ (lower three panels) are plotted for the three typical settings SB1 (left panel), MB1 (middle panel), and MB2 (right panel) defined in Sec. VIA. The true value of $\theta_{13}$ is taken as $\sin ^{2} 2 \theta_{13}=0.05$, which is indicated by the horizontal solid line in the figure.

$A / \Delta_{31} \simeq 0.06-0.07(0.2)$ for SB1 and MB2 (MB1) settings. Whereas for $\sin ^{2} 2 \theta_{13}^{\mathrm{II}}$ there is no small parameter which forces it small. The mild energy dependence and the pinning to a small value makes $\sin ^{2} 2 \theta_{13}^{\mathrm{III}}$ small in the entire region of $E$. The relatively fast reach to the asymptotic behavior can be seen in most of the plots of energy dependence of the degeneracy solutions for SB1 and MB1 settings. Notice, however, that the asymptotic energy can be reached at much higher energies for MB2 setting.

Here are comments on the solution $\left(s_{\mathrm{IV}}, \delta_{\mathrm{IV}}\right): R_{\mathrm{IV}}$ essentially looks like $R_{\mathrm{II}}$ apart from the presence of no-solution regions. Given smallness of $R_{\mathrm{III}}, R_{\mathrm{IV}}$ must looks like $R_{\mathrm{II}}$ because they are the intrinsic degeneracy pairs, as discussed in Sec. V. It is also true that the energy dependence of $\sin ^{2} 2 \theta_{13}^{\mathrm{IV}}$ is very similar to the behavior of $\sin ^{2} 2 \theta_{13}^{\mathrm{II}}$. Therefore, lifting degeneracy between $\theta_{13}^{\mathrm{IV}}$ and $\theta_{13}^{\mathrm{III}}$ can be done with spectrum analysis via a similar manner as in the case of intrinsic degeneracy. If powerful enough the spectrum informations would solve both the degeneracy between the true solution and $\theta_{13}^{\mathrm{II}}$, and the one between $\theta_{13}^{\mathrm{IV}}$ and $\theta_{13}^{\mathrm{III}}$ at the same time.

Next, we discuss $D_{\mathrm{III}}^{(2)}$ and $D_{\mathrm{IV}}^{(1)}$ which are presented in the upper and lower three panels, respectively, in Fig. 12. We note that they are small in SB1 setting, leaving the sign$\Delta m_{31}^{2}$ degeneracy intact in this short baseline setting. Notice, however, that it is not all bad, because the smallness of $D_{\text {III }}^{(2)}$ implies that no severe confusion takes place between $\mathrm{CP}$ violation and $\mathrm{CP}$ conservation. Now, the difference between SB1 and MB1 settings further develops in particular in large $\theta_{13}$ region. The clear distinction between SB1 and MB1 settings is also prominent in the energy dependence presented in Fig. 13 , Of course, it is basically due to larger matter effect in MB1 setting. It is interesting to observe that the difference shows up first in $\delta$, but not quite for $\theta_{13}$ at large $\theta_{13}$.

In the SB1 and MB1 settings, as can be seen in Fig. $12, D_{\mathrm{III}}^{(2)}$ and $D_{\mathrm{IV}}^{(1)}$ are largest in region of the largest possible $\theta_{13}$ for which the sign- $\Delta m_{31}^{2}$ degeneracy solution exist. In this region $\delta \sim \pi / 2$. On the other hand, $R_{\mathrm{III}}$ and $R_{\mathrm{IV}}$ are small in the region as is seen in 

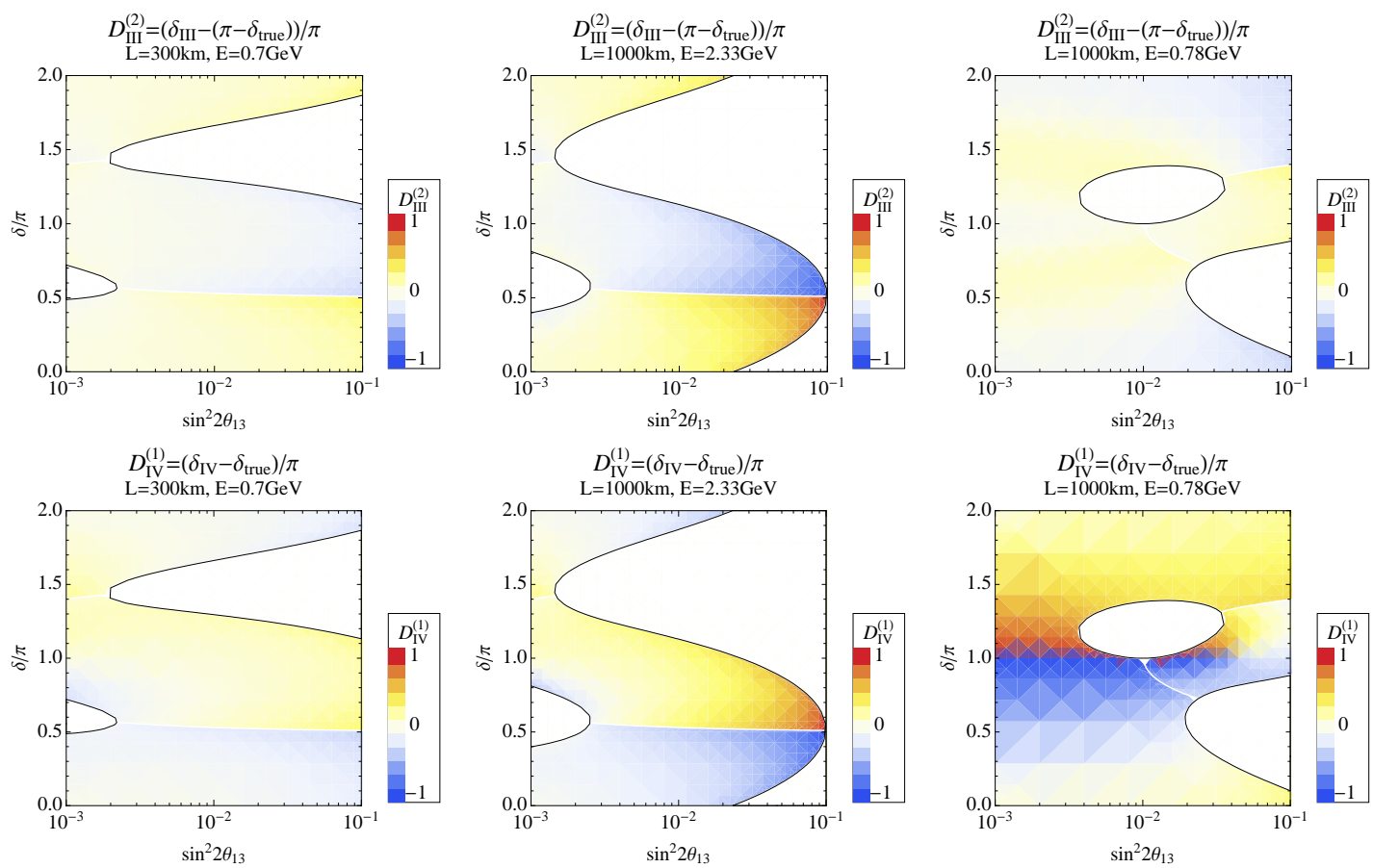

FIG. 12: $\quad D_{\text {III }}^{(2)} \equiv\left[\delta^{\mathrm{III}}-\left(\pi-\delta^{\text {true }}\right)\right] / \pi$ (upper three panels) and $D_{\mathrm{IV}}^{(1)} \equiv\left(\delta^{\mathrm{IV}}-\delta^{\text {true }}\right) / \pi$ (lower three panels) defined in 75 is presented in $\sin ^{2} 2 \theta_{13}-\delta / \pi$ space for the three typical settings SB1 (left panel), MB1 (middle panel), and MB2 (right panel) defined in Sec. VIA. The regions of white color denote the regions of no sign-degeneracy solution.
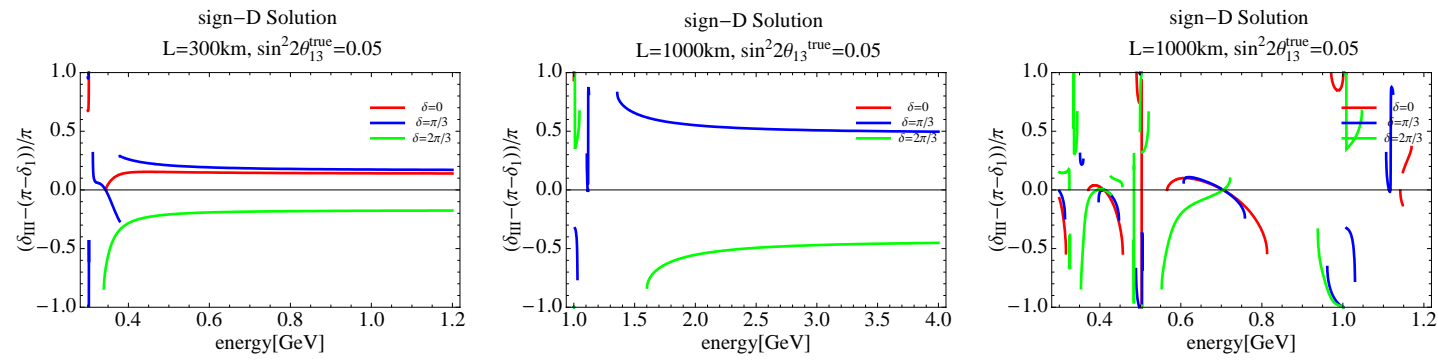

FIG. 13: The energy dependence of the ratios $D_{\text {III }}^{(2)}$ defined in 75 are plotted for the three typical settings SB1 (left panel), MB1 (middle panel), and MB2 (right panel) defined in Sec. VIA. $D_{\text {IV }}^{(1)}$ is not shown because the similarity in its energy dependence, apart from reversing the positive and negative regions of the ordinate. The true value of $\theta_{13}$ is taken as $\sin ^{2} 2 \theta_{13}=0.05$.

Fig. 10. It is easy to understand these features. At around the largest value of $\theta_{13}$ which allows the sign- $\Delta m_{31}^{2}$ degeneracy the two ellipses, the ones with normal and inverted mass hierarchies, barely overlap with each other. See Fig. 8 in [29]. The general feature of the bi-probability plot [24] tells us that in the overlap regions of the two ellipses the point of $\delta \sim \pi / 2$ in the positive $\Delta m_{31}^{2}$ ellipse is close to point of $\delta \sim 3 \pi / 2$ of the negative $\Delta m_{31}^{2}$ ellipse. Therefore, $D_{\text {III }}^{(2)} \simeq 1$ and $D_{\text {IV }}^{(1)} \simeq 1$ hold, explaining the above features. Because the center of the two ellipses are located at almost the same distances from the origin (which is 
equal to $\left.s_{23}^{2} \sin ^{2} 2 \theta_{13}\right), s_{\mathrm{III}} \simeq s_{\mathrm{IV}} \simeq s_{1}$.

We want to note that the energy dependences of $D_{\mathrm{III}}^{(2)}$ and $D_{\mathrm{IV}}^{(1)}$ are quite mild in energy region above the first oscillation maximum for SB1 and MB1 settings. Because the energy dependence of $D_{\mathrm{IV}}^{(1)}$ is similar to that of $D_{\mathrm{III}}^{(2)}$ in for SB1 and MB1 settings, apart from reversing the positive and negative regions of ordinate, we do not present it in Fig. 13. (Hereafter, we just quote the reference either [82] or [79] if the omitted figures are available in them.) For MB2 setting qualitative features of rapid up and down are very similar in all the figures of energy dependences presented, or abbreviated. Considering the almost no energy dependence of $\sin ^{2} 2 \theta_{13}^{\mathrm{III}}-\sin ^{2} 2 \theta_{13}^{\text {true }}$ as given in Fig. 11, and noting that spectrum analysis is highly challenging at low energies, it would be difficult to resolve the sign- $\Delta m_{31}^{2}$ degeneracy by a single detector setting of either SB1 or MB1.

We notice that the difference between SB1-MB1 and MB2 settings is always evident as can be seen in Figs. 10, 11, 12 and 13. Therefore, MB2 setting alone may have chance to resolve the sign- $\Delta m_{31}^{2}$ degeneracy [55, 56]. Or, if the informations gained at around the second oscillation maximum can somehow be combined it would greatly help resolving the sign- $\Delta m_{31}^{2}$ degeneracy [52 54, 57]. It may be expected even from our formulas obtained for a "mono-energetic neutrino beam" because the parameter regions with degeneracy solutions in SB1 and MB2 settings tend to "repel" (avoid to overlap) with each other at large $\theta_{13}$, though not completely.

Finally, we should note that abrupt termination of lines in the figures that appears in Figs. 11 and 13 are either due to disappearance of the degeneracy solutions, or switching phenomenon between solutions that takes place due to our convention of labeling degeneracy solutions. The feature will be seen also in the foregoing subsections. See Secs. IIID and IV for discussion on this point.

\section{Intrinsic and sign- $\Delta m_{31}^{2}$ degeneracies in the false $\theta_{23}$ octant}

Now, we turn to the $\theta_{23}$ octant degeneracy with solutions which lives in the different $\theta_{23}$ octant from the true solution. Having the overview at hand, we present the intrinsic and the sign- $\Delta m_{31}^{2}$ degeneracy solutions at the same time. Presented in Fig. 14 in $\sin ^{2} 2 \theta_{13}-\delta / \pi$ space are the ratios $R_{\mathrm{V}}=\left(\sin ^{2} 2 \theta_{13}^{\mathrm{V}}-\sin ^{2} 2 \theta_{13}^{\text {true }}\right) / \sin ^{2} 2 \theta_{13}^{\text {true }}$ (top three panels), $R_{\mathrm{VI}}$ (next to top panels), $R_{\mathrm{VII}}$ (next next to top panels), and $R_{\mathrm{VIII}}$ (bottom three panels) defined in (74) for three typical cases of energies and baselines, SB1 (left panels), MB1 (middle panels), and MB2 (right panels) defined in Sec. VIA. In Fig. 15, the energy dependence of degeneracy solutions of $\sin ^{2} 2 \theta_{13}^{\mathrm{V}}$ is presented. We note that the energy dependence of $\sin ^{2} 2 \theta_{13}^{\mathrm{VII}}$ is quite similar to that of $\sin ^{2} 2 \theta_{13}^{\mathrm{V}}$. The relation between the energy dependences of $\sin ^{2} 2 \theta_{13}^{\mathrm{V}}$ and $\sin ^{2} 2 \theta_{13}^{\mathrm{VI}}\left(\sin ^{2} 2 \theta_{13}^{\mathrm{VII}}\right.$ and $\left.\sin ^{2} 2 \theta_{13}^{\mathrm{VIII}}\right)$ is similar to the one between $\sin ^{2} 2 \theta_{13}^{\mathrm{III}}$ and $\sin ^{2} 2 \theta_{13}^{\mathrm{IV}}$ given in Fig. 11. (See [79, 82].) It is natural because they are the intrinsic degeneracy partners, and hence they are not shown.

One of the most notable features in Fig. 14 is again quite distinct behaviors in the MB2 setting. In general, $R_{\mathrm{N}}$ are large (apart from the strips where $R_{\mathrm{N}}$ switches its sign) with notable exceptions of $R_{\mathrm{V}}$ for SB1 and MB1 settings, and $R_{\mathrm{VII}}$ for SB1 setting. It is also notable that behavior of $R_{\mathrm{VI}}$ and $R_{\mathrm{VIII}}$ is reminiscent of the one of $R_{\mathrm{II}}$ in Fig. 6] in SB1 and MB1 settings in small $\theta_{13}$ region, except for the presence of no-solution region. Considering the small values of $R_{\mathrm{V}}$ and $R_{\mathrm{VII}}$ (except for $R_{\mathrm{VII}}$ for $\mathrm{MB} 1$ ), it is quite natural to see the

behavior given the fact that they are the intrinsic degeneracy partners. The feature that 
$R_{\mathrm{VI}}$ and $R_{\mathrm{VIII}}$ trace the behavior of their intrinsic degeneracy partners also applies to MB2 setting. The behavior of $R_{\mathrm{V}}$ and $R_{\mathrm{VII}}\left(R_{\mathrm{VI}}\right.$ and $\left.R_{\mathrm{VIII}}\right)$ is somewhat similar, apart from the presence of no-solution region, to that of $R_{\mathrm{II}}$ for SB1 (MB2) setting. It may be understood by the similar consideration using the bi-probability plot. Therefore, we concentrate below on SB1 and MB1 settings.

As mentioned above $R_{\mathrm{V}}$ for SB1 and MB1 settings, and $R_{\mathrm{VII}}$ for SB1 setting are small in region $\sin ^{2} 2 \theta_{13} \gtrsim 10^{-2}$. The region of $\theta_{13}$, however, is nothing but a good target for superbeam experiments, and it will be a challenge for them to lift the degeneracy solutions. Here, we try to understand this feature, but in a wider perspective which includes the energy dependence of $\sin ^{2} 2 \theta_{13}^{\mathrm{V}}$ and $\sin ^{2} 2 \theta_{13}^{\mathrm{VII}}$ for SB1 and MB1 settings. In Fig. 15, we observe that the difference between $\sin ^{2} 2 \theta_{13}^{\mathrm{V}}$ (or, $\sin ^{2} 2 \theta_{13}^{\mathrm{VII}}$ ) and $\sin ^{2} 2 \theta_{13}^{\text {true }}$ is nonzero but energy independent in a wide region except for at very low energies, a somewhat unexpected behavior to see.

Now, we point out that the behaviors mentioned above can be understood by formulating the $\theta_{23}$ perturbation theory, as done in Appendix B. Namely, one can derive the perturbative expression of $\sin ^{2} 2 \theta_{13}^{\mathrm{N}}$ and other quantities by assuming that deviation of $\theta_{23}$ from $\pi / 4$ is small, $\theta_{23}-\pi / 4 \equiv \epsilon_{\text {oct }} \ll 1$. One can expect that the expansion by $\epsilon_{\text {oct }}$ is indeed a good approximation because e.g., $\epsilon_{\text {oct }}=0.05$ for $\theta_{23}=42^{\circ}$. Then, we obtain

$$
\sin ^{2} 2 \theta_{13}^{\mathrm{V}}-\sin ^{2} 2 \theta_{13}^{\text {true }}=4 \epsilon_{\text {oct }} \sin ^{2} 2 \theta_{13}^{\text {true }}
$$

for which we have used the fact that the last term in $(\overline{\mathrm{B} 2})$ is negligibly small as far as we remain in a region $\sin ^{2} 2 \theta_{13}^{\text {true }} \gg Z$. In fact, we confirmed that the correction term becomes non-negligible at small $\theta_{13}$ around $\sin 2 \theta_{13}^{\text {true }}=10^{-3}$. Certainly, the condition is fulfilled for the settings SB1 and MB1. The similar equation holds for $\theta_{13}^{\mathrm{VII}}$ but with replacement of $\theta_{13}^{\text {true }}$ to $\theta_{13}^{\mathrm{III}}$ because their relation as the intrinsic degeneracy partner. Therefore, $R_{\mathrm{V}}$ and $R_{\mathrm{VII}}$ are small and the difference in (76) is approximately energy independent. One may ask why the feature does not exist in MB2 setting with small $\theta_{13}$. As mentioned before, the solar term is dominant in this region. Therefore, if $\epsilon_{\mathrm{oct}} Y_{ \pm}$is comparable to $s_{1}$ the difference $s_{\mathrm{V}}-s_{1}$ is no more small.

Another notable point is that region of no degeneracy solution is not additive, as can be seen by comparing Figs. 10 and 14. That is, the region of no degeneracy solution with $\Delta m_{31}^{2}$-sign and $\theta_{23}$ octant flips (VII and VIII) is not the union of no-solution regions of the sign- $\Delta m_{31}^{2}$ (III) and the $\theta_{23}$ octant (V) degeneracies. It is simply because the degeneracy solution with both sign and octant flips can exist even in a region of $\theta_{13}$ and $\delta$ where e.g., the octant degeneracy solution does not exist.

In Fig. 16, from the top to the bottom, the normalized differences between the true and fake solutions of phases, $D_{\mathrm{V}}^{(1)}, D_{\mathrm{VI}}^{(2)}, D_{\mathrm{VII}}^{(2)}$, and $D_{\mathrm{VIII}}^{(1)}$ defined in 75 are presented for SB1 (left panels), MB1 (middle panels), MB2 (right panels) settings. In Fig. 17, the energy dependence of $D_{\mathrm{V}}^{(1)}$ is plotted. The behavior of $D_{\mathrm{VI}}^{(2)}$ is similar to $D_{\mathrm{II}}^{(2)}$ given in Fig. 9 , while those of $D_{\mathrm{VII}}^{(2)}$ and $D_{\mathrm{VIII}}^{(1)}$ are very similar to the ones of $D_{\mathrm{III}}^{(2)}$ and $D_{\mathrm{IV}}^{(1)}$ (the latter not shown but the behavior explained) in Fig. 13 . See [79, 82. We notice that for SB1 setting $D_{\mathrm{N}}^{(i)}(i$ either 1 or 2) is small in most of the regions of true values of $\delta$ for all the solutions V-VIII. For MB1 setting the same statement applies for the solutions V and VI. A notable feature is that $D_{\mathrm{V}}^{(1)}$ (and $D_{\mathrm{VII}}^{(2)}$ ) is small in MB2 setting. It can also be understood from the $\theta_{23}$ perturbative formula for $\delta$ given in Appendix B; The difference between $\delta_{1}$ and $\delta_{\mathrm{V}}$ is always suppressed by $\epsilon_{\text {oct }}$. 

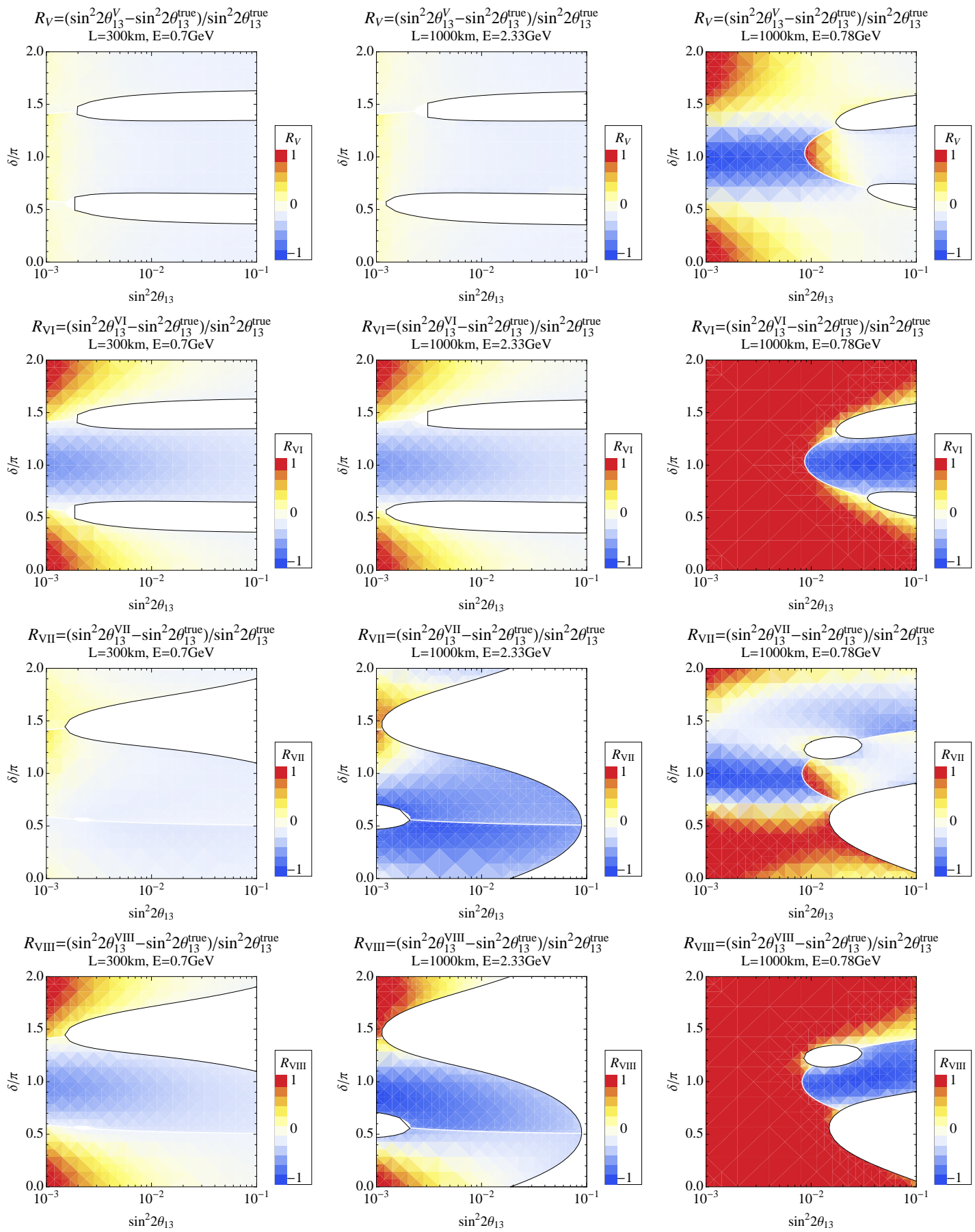

FIG. 14: Plotted are the ratios $R_{\mathrm{V}}=\left[\sin ^{2} 2 \theta_{13}^{\mathrm{V}}-\sin ^{2} 2 \theta_{13}^{\text {true }}\right] / \sin ^{2} 2 \theta_{13}^{\text {true }}$ (top three panels), $R_{\mathrm{VI}}$ (next to top panels), $R_{\mathrm{VII}}$ (next next to top panels), and $R_{\mathrm{VIII}}$ (bottom three panels) defined in 74 in $\sin ^{2} 2 \theta_{13}-\delta / \pi$ space is presented for three typical cases of energies and baselines, SB1 (left panels), MB1 (middle panels), and MB2 (right panels) defined in Sec. VIA. The regions of white color denote the regions of no degeneracy solution. 

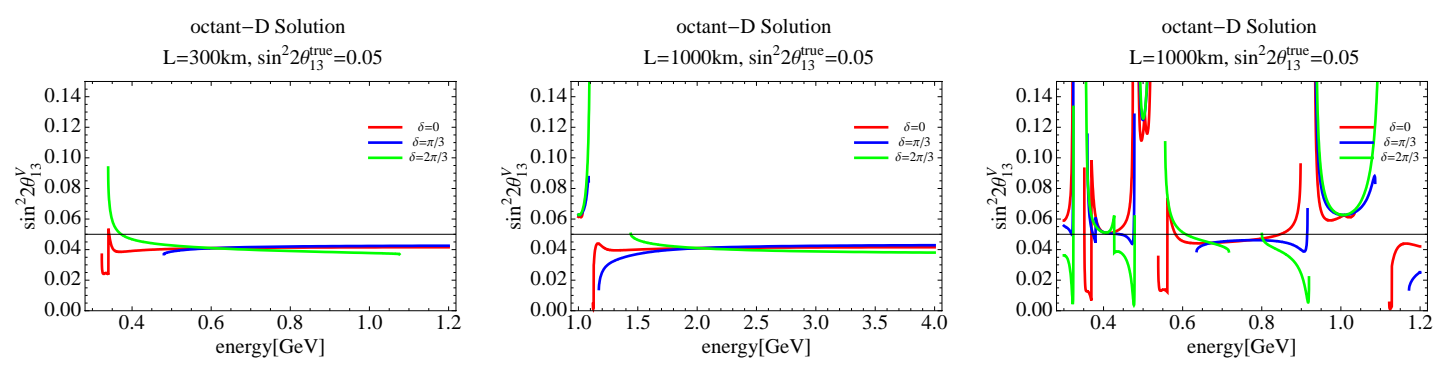

FIG. 15: The energy dependence of $\sin ^{2} 2 \theta_{13}^{\mathrm{V}}$ are plotted for the three typical settings SB1 (left panel), MB1 (middle panel), and MB2 (right panel) defined in Sec. VIA. For the behavior of the other solutions not shown, $\sin ^{2} 2 \theta_{13}^{\mathrm{VI}}, \sin ^{2} 2 \theta_{13}^{\mathrm{VII}}$, and $\sin ^{2} 2 \theta_{13}^{\mathrm{VIII}}$, see the text. The true value of $\theta_{13}$ is taken as $\sin ^{2} 2 \theta_{13}=0.05$, which is indicated by the horizontal solid line in the figure. The true value of $\theta_{23}$ is $42^{\circ}$.

As discussed above the energy dependence is very mild for most of the solutions V-VIII, except for at low energies, $E \lesssim 0.4 \mathrm{GeV}$, in SB1 and MB1 settings. Therefore, it may be extremely challenging for experiments with the settings to lift the degeneracy. Because of the likely difficulty in resolving the $\theta_{23}$ octant degeneracy several methods have been proposed; the reactor-accelerator combined method [8, 58, the various ways to detect solar $\Delta m_{21}^{2}$ scale oscillations, using atmospheric [59 62] or accelerator neutrinos [54 56], or both combined [51, 63]. The silver channel could be of help [64]. As in the previous cases the behavior of degeneracy solutions are far more violent in MB2 setting. It by itself might mean the great sensitivity to resolve the degeneracy. Or, it is a natural way of thinking to combine it with the measurement at the first oscillation maximum.

We give here a brief summary of the characteristic features of the degeneracy in superbeams, SB1, MB1, and MB2 settings.

- A prominent difference between the true and the clone solutions at relatively large $\theta_{13}, \sin ^{2} 2 \theta_{13} \gtrsim 10^{-2}$, exists in $R_{\mathrm{N}}$ in the intrinsic degeneracy, while for the sign- $\Delta m_{31}^{2}$ degeneracy it is in the phase difference $D_{\mathrm{N}}^{(i)}$ for SB1 and MB1 settings.

- The solutions III for the sign- $\Delta m_{31}^{2}$ degeneracy appears to be difficult to resolve for SB1 setting even if spectrum information is available, because energy dependences are so weak for both $R_{\mathrm{III}}$ and $D_{\mathrm{III}}^{(2)}$. The similar difficulty exists for MB1 if $\theta_{13}$ is large, $\sin ^{2} 2 \theta_{13} \gtrsim 10^{-2}$. For the same reason, the solutions V and VII of the $\theta_{23}$ octant degeneracy is difficult to lift.

- The short baseline SB1 option is unique among the three superbeam settings in the sense that it by itself may not be able to lift the sign- $\Delta m_{31}^{2}$ and the $\theta_{23}$ octant degeneracies, but can provide a clean discovery of $\mathrm{CP}$ violation without confusion with $\mathrm{CP}$ conservation. This is in accord with the basic motivation for low energy superbeam [66].

- In comparison with SB1 and MB1 settings, the features of degeneracy solutions are always quite distinct at MB2 setting, where the energy region around the second oscillation maximum is explored. It by itself, or combined with other settings, would provide ways to help resolving the eightfold degeneracy. 

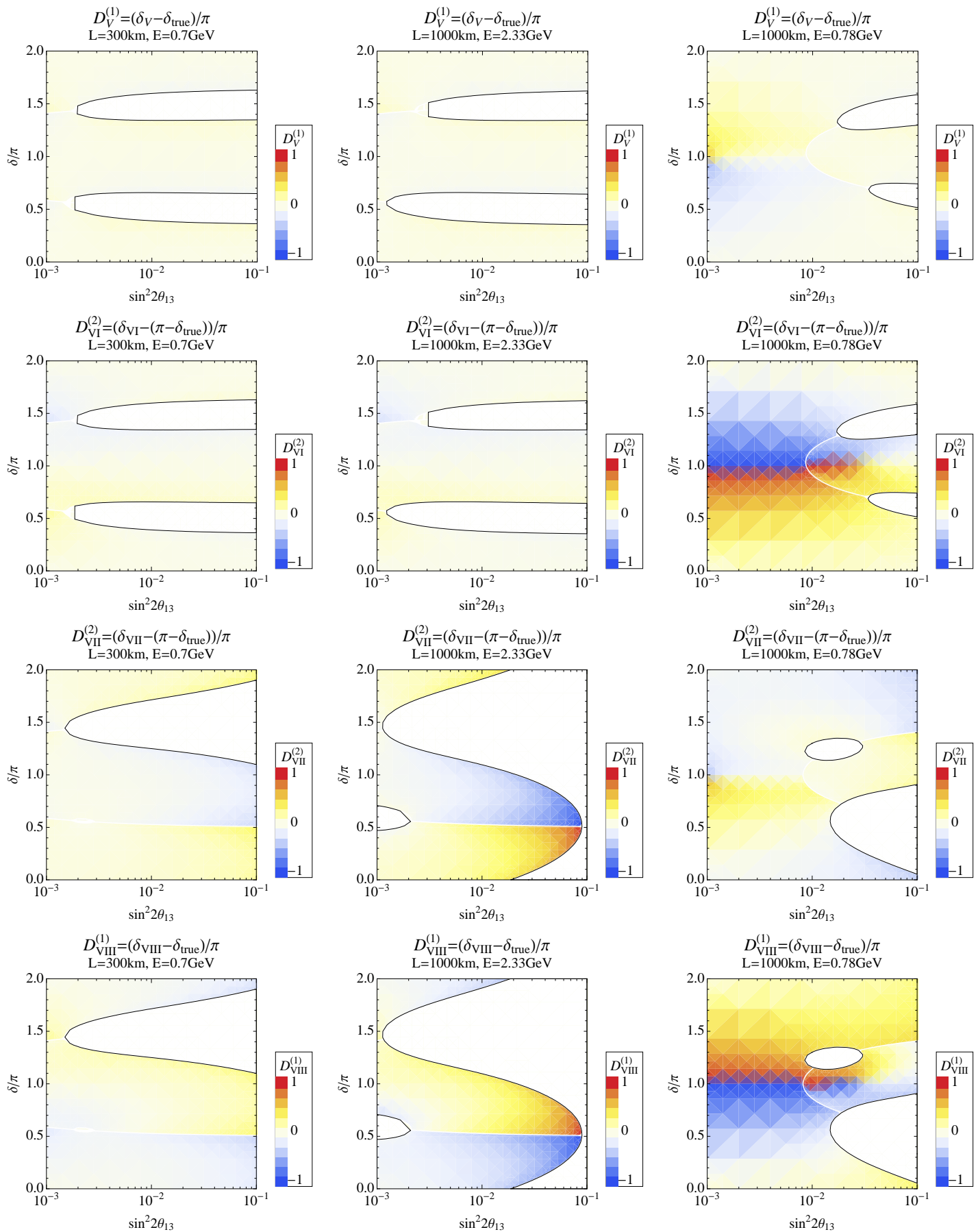

FIG. 16: From top panels to bottom panels presented are in order: $D_{\mathrm{V}}^{(1)} \equiv\left(\delta^{\mathrm{V}}-\delta^{\text {true }}\right) / \pi$, $D_{\mathrm{VI}}^{(2)} \equiv\left[\delta^{\mathrm{VI}}-\left(\pi-\delta^{\text {true }}\right)\right] / \pi, D_{\mathrm{VII}}^{(2)} \equiv\left[\delta^{\mathrm{VII}}-\left(\pi-\delta^{\text {true }}\right)\right] / \pi$, and $D_{\mathrm{VIII}}^{(1)} \equiv\left(\delta^{\mathrm{VIII}}-\delta^{\text {true }}\right) / \pi$ defined in 75. for three typical cases of energies and baselines in $\sin ^{2} 2 \theta_{13}^{\text {true }}-\delta^{\text {true }} / \pi$ space. The regions of white color denote the regions of no sign-degeneracy solution. 

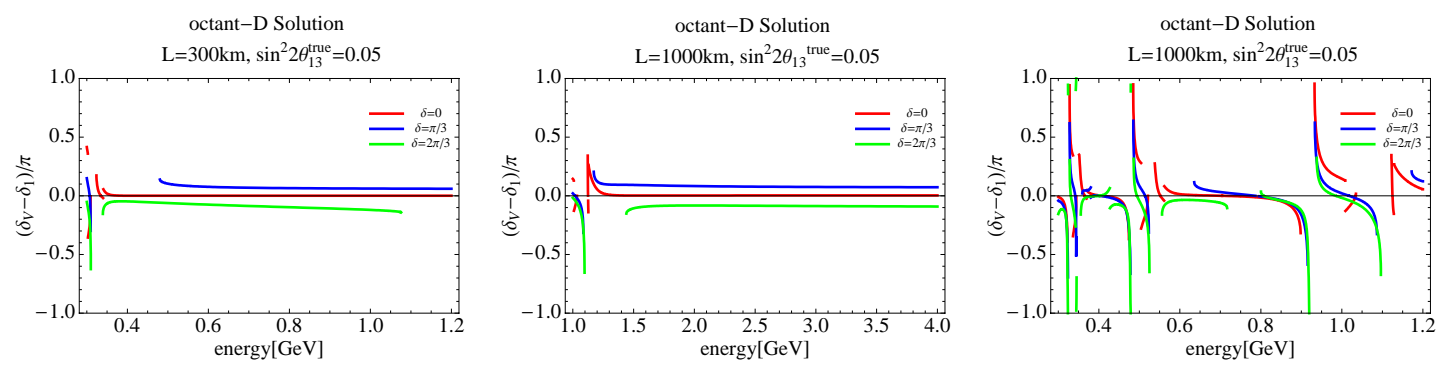

FIG. 17: The energy dependence of the ratio $D_{\mathrm{V}}^{(1)}$ defined in 75 are plotted for the three typical settings SB1 (left panel), MB1 (middle panel), and MB2 (right panel) defined in Sec. VIA. For the behavior of the other solutions not shown, $D_{\mathrm{VI}}^{(2)}, D_{\mathrm{VII}}^{(2)}$, and $D_{\mathrm{VIII}}^{(1)}$, see the text. The true value of $\theta_{13}$ is taken as $\sin ^{2} 2 \theta_{13}=0.05$.

\section{E. Parameter degeneracy in neutrino factory setting}

In this subsection, we display the features of various degeneracy solutions by taking a setting which may be appropriate for neutrino factory. The two-detector setting with baselines $L=3000-4000 \mathrm{~km}$ and $L \sim 7000 \mathrm{~km}$ seems to be considered as the "standard" one [83] both for measurement of standard mixing parameters [23, 84], possibly as well as for search for effects of NSI [39, 85, 86. For the former purpose, the far detector at the "magic baseline" (as named by [84]) plays a key role in resolving the degeneracy because of absence of $\delta$ dependence [27]. By restricting our purpose to illuminate the features of the degeneracy, we use the setup with just one detector at $L=4000 \mathrm{~km}$.

Note that we have used set of probabilities $P\left(\nu_{\mu} \rightarrow \nu_{e}\right)$ and $P\left(\bar{\nu}_{\mu} \rightarrow \bar{\nu}_{e}\right)$ to obtain the degenerate solutions. Therefore, if you want to consider the more realistic setting of a neutrino factory in which T-conjugate (golden) channels will be used, please regard $\delta$ as $2 \pi-\delta$.

In Figs. 18 and 19, the differences between the true solution and the fake degeneracy solutions, $R_{\mathrm{N}}$ and $D_{\mathrm{N}}^{(i)}(\mathrm{N}=\mathrm{II}-\mathrm{VIII}, i=1$ or 2$)$, respectively, are plotted. As can be seen in these figures the differences between the true solution and the fake degeneracy solutions are generically larger than the cases of superbeam type settings discussed in the previous subsections. In accord with the expected higher sensitivities, we extend the region of $\sin ^{2} 2 \theta_{13}$ to $10^{-4}$.

In Figs. 20 and 21, the energy dependences of $\sin ^{2} 2 \theta_{13}^{\mathrm{N}}$ and $D_{\mathrm{N}}^{(i)}$, respectively, are presented for three representative solutions, II, III, and V. For energy dependences of the other solutions see [79, 82]. Generally speaking, the energy dependences of both of the quantities are significant compared to those in the SB1 and MB1 settings. Notable exceptions are the solution $\mathrm{V}$ (both $\sin ^{2} 2 \theta_{13}^{\mathrm{V}}$ and $D_{\mathrm{V}}^{(1)}$ ), and possibly $\sin ^{2} 2 \theta_{13}^{\mathrm{III}}$ and $\sin ^{2} 2 \theta_{13}^{\mathrm{VII}}$, all except for the low energy region $E \lesssim 10 \mathrm{GeV}$. Unless there is a sensitivity to the low-energy region it would be difficult to resolve the degeneracy, in particular $\mathrm{V}$, by the spectrum informations. Therefore, it is extremely important to lower the threshold into $E \lesssim 10 \mathrm{GeV}$ to resolve the degeneracy by spectrum analysis. An extensive effort toward this direction is made and the task is in progress [87.

It is possible to understand, at least qualitatively, smallness of the difference from the true solution and lack of strong energy dependence of the solutions V and VII. Because the $\theta_{23}$ perturbation theory also applies to NF setting, it can be expected that the difference 

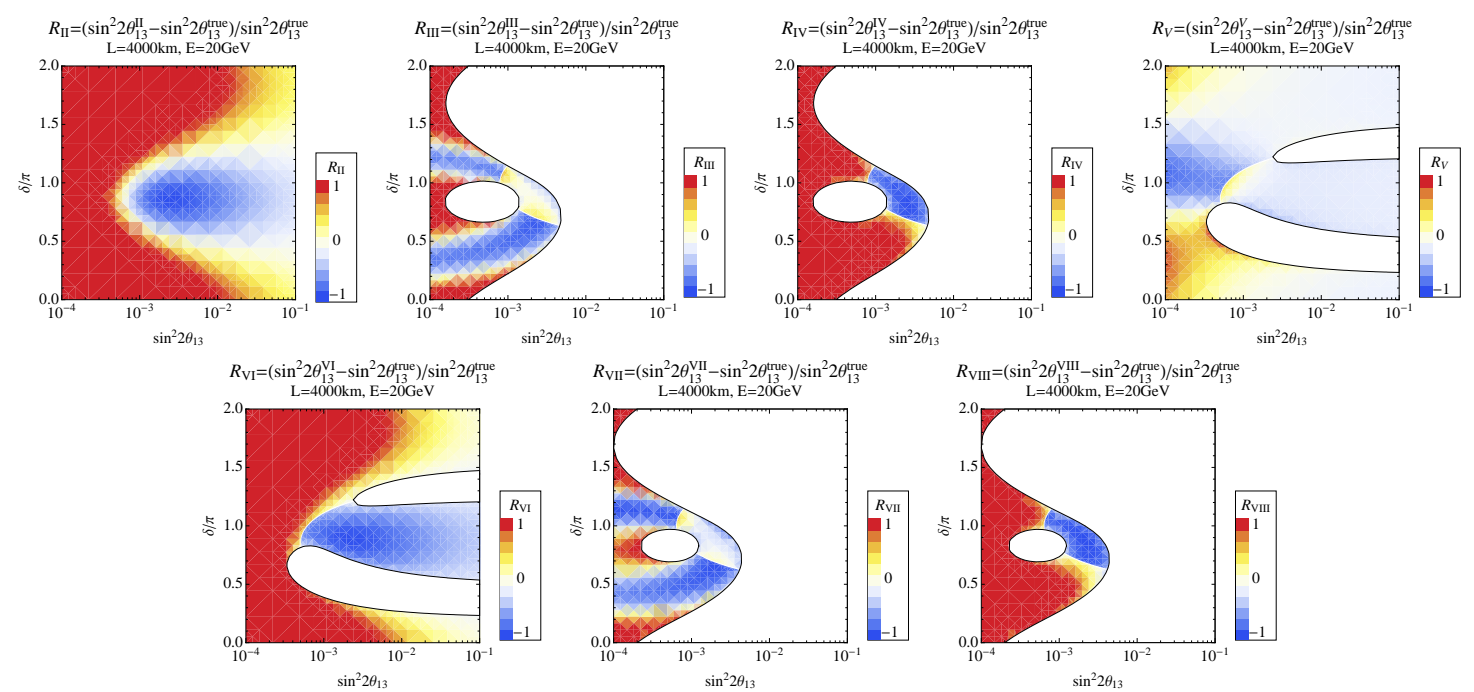

FIG. 18: The ratio $R_{\mathrm{II}}-R_{\mathrm{VIII}}$ are plotted in $\sin ^{2} 2 \theta_{13}-\delta / \pi$ space for a typical baseline and energy of neutrino factory setting. The ratio $R_{\mathrm{N}}$ is defined as $R_{\mathrm{N}}=\left[\sin ^{2} 2 \theta_{13}^{\mathrm{N}}-\sin ^{2} 2 \theta_{13}^{\text {true }}\right] / \sin ^{2} 2 \theta_{13}^{\text {true }}$ defined in $(74)$
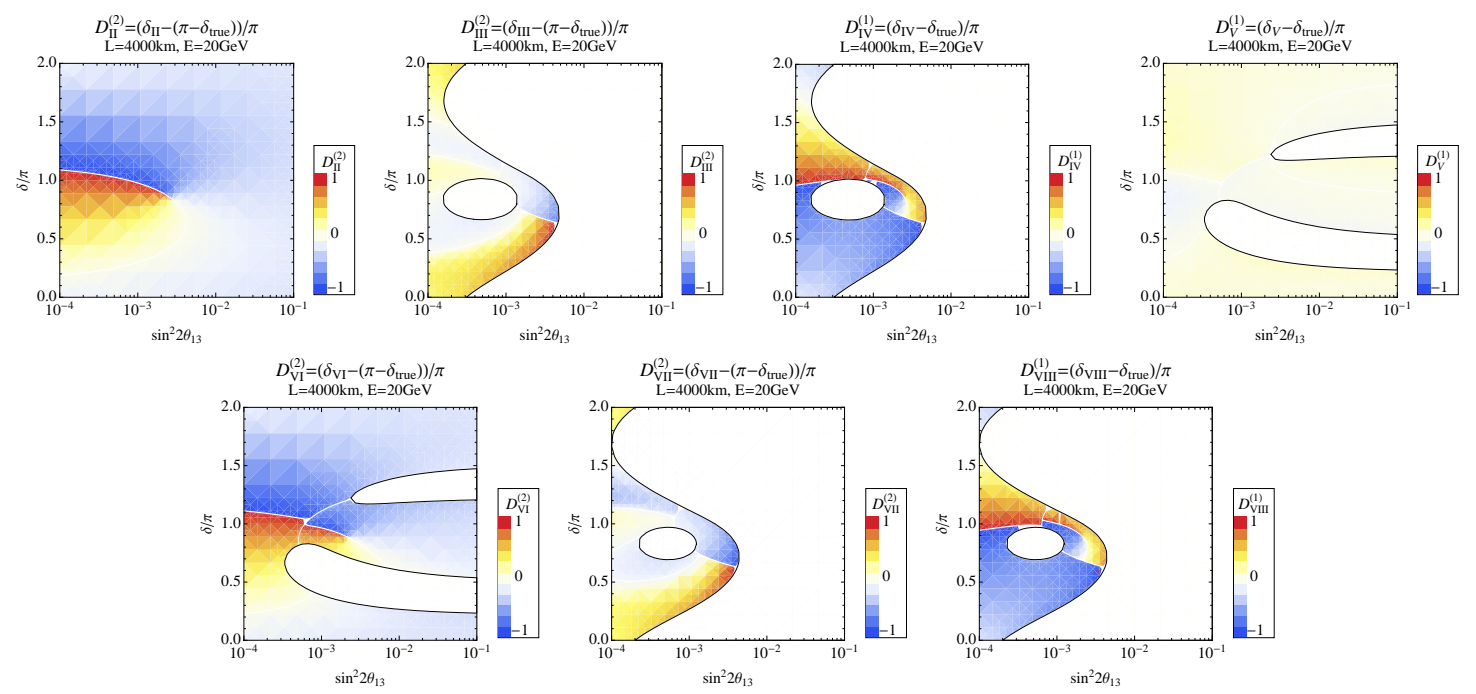

FIG. 19: The normalized differences $D_{\mathrm{II}}^{(2)}, D_{\mathrm{III}}^{(2)}, D_{\mathrm{IV}}^{(1)}, D_{\mathrm{V}}^{(1)}, D_{\mathrm{VI}}^{(2)}, D_{\mathrm{VII}}^{(2)}$, and $D_{\mathrm{VIII}}^{(1)}$ are plotted in order in $\sin ^{2} 2 \theta_{13}-\delta / \pi$ space for a typical baseline and energy of neutrino factory setting. $D_{\mathrm{N}}$ is defined in 75 as $D_{\mathrm{N}}^{(2)} \equiv\left[\delta^{\mathrm{N}}-\left(\pi-\delta^{\text {true }}\right)\right] / \pi$ and $D_{\mathrm{N}}^{(1)} \equiv\left[\delta^{\mathrm{N}}-\delta^{\text {true }}\right] / \pi$.

between the true solution and the clone one $\mathrm{V}$ is small. The similar statement holds for the solution VII given the smallness of the energy dependent term in $\sin ^{2} 2 \theta_{13}^{\text {III }}$. Then, the question is why $\sin ^{2} 2 \theta_{13}^{\text {III }}$ is small and lacks the significant energy dependence despite that the matter perturbation theory is not valid for NF setting. Qualitatively, the answer is that pinning to a small value due to the fact that the assumed true value itself is small, and lack of energy dependence because of fast reach to the asymptotic behavior discussed in Sec. V C. 

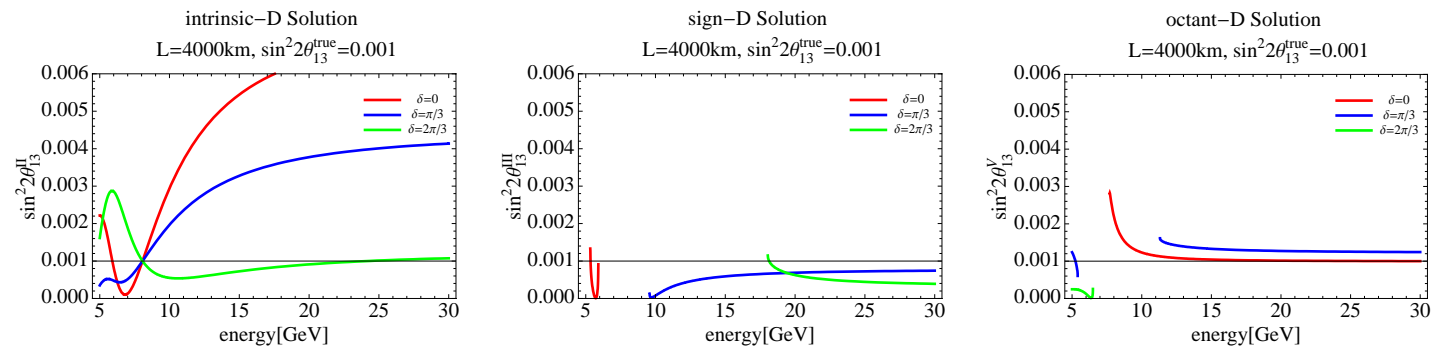

FIG. 20: The energy dependences of $\sin ^{2} 2 \theta_{13}^{\mathrm{II}}$ (left panel), $\sin ^{2} 2 \theta_{13}^{\mathrm{III}}$ (middle panel), and $\sin ^{2} 2 \theta_{13}^{\mathrm{V}}$ (right panel) are plotted. The true value of $\theta_{13}$ is taken $\operatorname{as}^{2} \sin ^{2} 2 \theta_{13}=0.001$, which is indicated by the horizontal solid line in the figure.
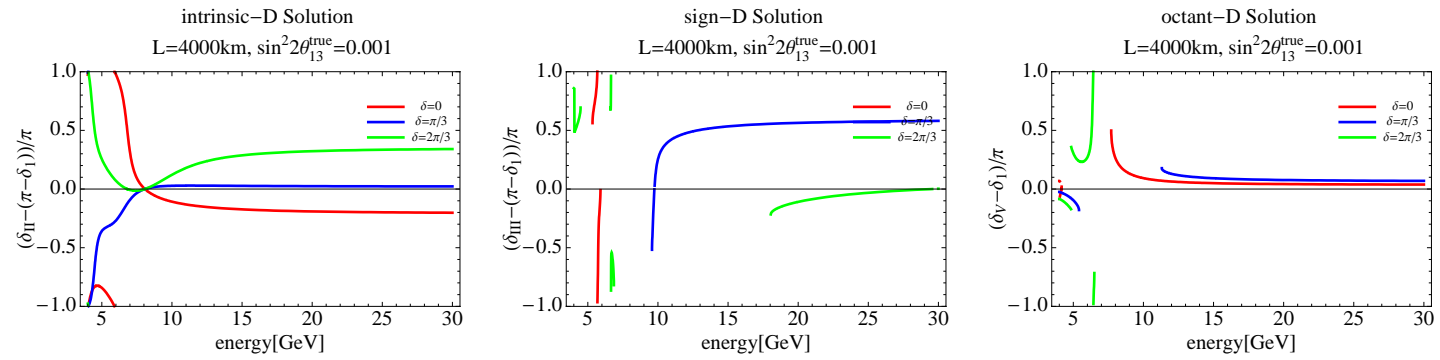

FIG. 21: The energy dependences of $D_{\mathrm{II}}^{(2)}$ (left panel), $D_{\mathrm{III}}^{(2)}$ (middle panel), and $D_{\mathrm{V}}^{(1)}$ (right panel) are plotted. $D_{\mathrm{N}}^{(1)}$ and $D_{\mathrm{N}}^{(2)}$ are defined in 75 .

One notices that the intrinsic solution II in NF setting has the similar features as the one in MB2 setting, as can be seen by comparing Figs. 18 and 19 to Figs. 5 and 6 . It is because the value of $\theta_{13}$ taken is small and the atmospheric term is comparable to the solar term. Parallelism is not so complete in the other types of degeneracies, but some features can be understood in analogy to the case of MB2 setting. ${ }^{14}$ In doing so the difference due to the much wider no-solution region of the sign- $\Delta m_{31}^{2}$ degeneracy due to long baseline must, of course, be taken into account.

\section{PARAMETER DEGENERACY IN T-CONJUGATE MEASUREMENT}

We analyze in this section the problem of parameter degeneracy in T-conjugate measurement in neutrino oscillation. Though measurement of $\mathrm{T}$ violation does not appear to be feasible immediately understanding its structure may be interesting theoretically. The topics was first treated in [29], but we make the structure of the degeneracy more transparent in harmony with the symmetry argument in Sec. II B. In fact, the structure of the degeneracy with T-conjugate measurement is one of the simplest one among the cases discussed due to

14 An example is that there is the region that $R_{\mathrm{V}}$ is large in small $\theta_{13}$. This is for the same reason of the case in MB2 setting, the difference between the true and $\mathrm{V}$ of order $\sim \epsilon_{\mathrm{oct}} Y_{ \pm}$, is negligible compare with $s_{1}$. Another example is much stronger energy dependence of $s_{\text {II }}$ in NF setting than SB1's, which is reminiscent of the feature of MB2 setting. It comes from larger effect of the solar-scale oscillation term. 
the symmetry. It is also an ideal tool to obtain the vacuum limit.

\section{A. The intrinsic degeneracy in T-conjugate measurement}

The intrinsic degeneracy solutions $\left(s_{i}, \delta_{i}\right)(\mathrm{i}=1,2)$ are defined in $\nu_{\mu} \rightarrow \nu_{e}$ channel by

$$
\begin{aligned}
& P-Z=X_{ \pm} s_{1}^{2}+Y_{ \pm} s_{1}\left(\cos \delta_{1} \cos \Delta_{31} \mp \sin \delta_{1} \sin \Delta_{31}\right), \\
& P-Z=X_{ \pm} s_{2}^{2}+Y_{ \pm} s_{2}\left(\cos \delta_{2} \cos \Delta_{31} \mp \sin \delta_{2} \sin \Delta_{31}\right),
\end{aligned}
$$

and in T-conjugate channel by

$$
\begin{aligned}
& P^{T}-Z=X_{ \pm} s_{1}^{2}+Y_{ \pm} s_{1}\left(\cos \delta_{1} \cos \Delta_{31} \pm \sin \delta_{1} \sin \Delta_{31}\right) \\
& P^{T}-Z=X_{ \pm} s_{2}^{2}+Y_{ \pm} s_{2}\left(\cos \delta_{2} \cos \Delta_{31} \pm \sin \delta_{2} \sin \Delta_{31}\right) .
\end{aligned}
$$

By subtracting two equations in (77) and (78), respectively, and then subtracting and adding the resultant two equations, we obtain (assuming $Y_{ \pm} \sin \Delta_{31} \neq 0$ )

$$
\begin{aligned}
s_{2} \sin \delta_{2} & =s_{1} \sin \delta_{1}, \\
s_{2} \cos \delta_{2} & =s_{1} \cos \delta_{1}+\frac{X_{ \pm}}{Y_{ \pm} \cos \Delta_{31}}\left(s_{1}^{2}-s_{2}^{2}\right) .
\end{aligned}
$$

Inserting (79) into $\cos ^{2} \delta_{2}+\sin ^{2} \delta_{2}=1$ yields the equation for $s_{2}$ in a form $\left(s_{2}^{2}-s_{1}^{2}\right)\left(s_{2}^{2}-s_{\text {II }}^{2}\right)=$ 0 , which admits the intrinsic degeneracy solution

$$
s_{\mathrm{II}}=\sqrt{s_{1}^{2}+2\left(\frac{Y_{ \pm} \cos \Delta_{31}}{X_{ \pm}}\right) s_{1} \cos \delta_{1}+\left(\frac{Y_{ \pm} \cos \Delta_{31}}{X_{ \pm}}\right)^{2}} .
$$

Given the solution $s_{2}=s_{\text {II }}$ the solution for $\delta_{2}$ can be obtained by using $(79)$ as

$$
\begin{aligned}
& s_{\mathrm{II}} \sin \delta_{\mathrm{II}}=s_{1} \sin \delta_{1}, \\
& s_{\mathrm{II}} \cos \delta_{\mathrm{II}}=-\left(s_{1} \cos \delta_{1}+\frac{Y_{ \pm} \cos \Delta_{31}}{X_{ \pm}}\right) .
\end{aligned}
$$

By further expanding by $\frac{Y_{ \pm}}{X}$, assuming it small, the solution obtained in [29] is reproduced;

$$
s_{\mathrm{II}} \simeq s_{1}+\frac{Y_{ \pm} \cos \Delta_{31}}{X_{ \pm}} \cos \delta_{1} .
$$

\section{B. The sign- $\Delta m^{2}$ degeneracy in T-conjugate measurement}

As we learned in Sec. IIB the symmetry argument tells us that there exists the sign$\Delta m^{2}$ degeneracy in T-conjugate measurement. In this subsection we verify it by deriving explicit solutions without recourse to the symmetry argument. We denote $s_{13}$ variable for the opposite-sign $\Delta m_{31}^{2}$ solution as $\left(s_{3}, \delta_{3}\right)$, whose two (as we prove) solutions will be denoted as $\left(s_{\mathrm{III}}, \delta_{\mathrm{III}}\right)$ and $\left(s_{\mathrm{IV}}, \delta_{\mathrm{IV}}\right)$.

The sign- $\Delta m_{31}^{2}$ degeneracy is defined by the following two sets of equations:

$$
\begin{aligned}
& P-Z=X_{ \pm} s_{1}^{2}+Y_{ \pm} s_{1}\left(\cos \delta_{1} \cos \Delta_{31} \mp \sin \delta_{1} \sin \Delta_{31}\right), \\
& P-Z=X_{\mp} s_{3}^{2}+Y_{\mp} s_{3}\left(\cos \delta_{3} \cos \Delta_{31} \pm \sin \delta_{3} \sin \Delta_{31}\right),
\end{aligned}
$$


and

$$
\begin{aligned}
& P^{T}-Z=X_{ \pm} s_{1}^{2}+Y_{ \pm} s_{1}\left(\cos \delta_{1} \cos \Delta_{31} \pm \sin \delta_{1} \sin \Delta_{31}\right) \\
& P^{T}-Z=X_{\mp} s_{3}^{2}+Y_{\mp} s_{3}\left(\cos \delta_{3} \cos \Delta_{31} \mp \sin \delta_{3} \sin \Delta_{31}\right) .
\end{aligned}
$$

By the similar procedure as in the previous subsection we obtain (assuming $Y_{ \pm} \sin \Delta_{31} \neq 0$ )

$$
\begin{aligned}
& s_{3} \sin \delta_{3}=-\left(\frac{Y_{ \pm}}{Y_{\mp}}\right) s_{1} \sin \delta_{1}, \\
& s_{3} \cos \delta_{3}=\left(\frac{Y_{ \pm}}{Y_{\mp}}\right) s_{1} \cos \delta_{1}+\frac{1}{Y_{\mp} \cos \Delta_{31}}\left(X_{ \pm} s_{1}^{2}-X_{\mp} s_{3}^{2}\right) .
\end{aligned}
$$

Inserting 85 into $\cos ^{2} \delta_{3}+\sin ^{2} \delta_{3}=1$ leads to the equation for $s_{3}^{2}$ as

$$
\left(s_{3}^{2}-\frac{X_{ \pm}}{X_{\mp}} s_{1}^{2}\right)\left(s_{3}^{2}-\frac{X_{ \pm}}{X_{\mp}} s_{\mathrm{II}}^{2}\right)=0,
$$

where $s_{\mathrm{II}}$ is defined in $(80)$. We note that the relation $(9)$ is essential to reduce the equation in (86) to the current form. The solutions for CP phase $\delta$ can be obtained by inserting the solutions of $(86)$, its positive root, into (85) with use of $(9)$, and for $\delta_{\text {IV }}$ together with (79). The solutions of $\delta$ together with the $\theta_{13}$ solutions of the sign- $\Delta m_{31}^{2}$ degeneracy are summarized as below:

$$
\begin{aligned}
& s_{\mathrm{III}}=\sqrt{\frac{X_{ \pm}}{X_{\mp}}} s_{1}, \quad \delta_{\mathrm{III}}=\pi-\delta_{1}, \\
& s_{\mathrm{IV}}=\sqrt{\frac{X_{ \pm}}{X_{\mp}}} s_{\mathrm{II}}, \quad \delta_{\mathrm{IV}}=\pi-\delta_{\mathrm{II}} .
\end{aligned}
$$

The solutions (87) are in perfect agreement with the expectation based on invariance of the oscillation probability given in Sec. IIB. Figure 2 clearly exhibits the structure obtained in (87).

\section{The octant degeneracy in T-conjugate measurement}

The $\theta_{23}$ octant degeneracy in T-conjugate measurement is defined by

$$
\begin{aligned}
P & =X_{ \pm}^{\text {true }} s_{1}^{2}+Y_{ \pm} s_{1}\left(\cos \delta_{1} \cos \Delta_{31} \mp \sin \delta_{1} \sin \Delta_{31}\right)+Z^{\text {true }} \\
P & =X_{ \pm}^{\text {false }} s_{5}^{2}+Y_{ \pm} s_{5}\left(\cos \delta_{5} \cos \Delta_{31} \mp \sin \delta_{5} \sin \Delta_{31}\right)+Z^{\text {false }} \\
P^{T} & =X_{ \pm}^{\text {true }} s_{1}^{2}+Y_{ \pm} s_{1}\left(\cos \delta_{1} \cos \Delta_{31} \pm \sin \delta_{1} \sin \Delta_{31}\right)+Z^{\text {true }} \\
P^{T} & =X_{ \pm}^{\text {false }} s_{5}^{2}+Y_{ \pm} s_{5}\left(\cos \delta_{5} \cos \Delta_{31} \pm \sin \delta_{5} \sin \Delta_{31}\right)+Z^{\text {false }}
\end{aligned}
$$

From these equations, we obtain

$$
\begin{aligned}
s_{5} \cos \delta_{5} & =s_{1} \cos \delta_{1}+\frac{1}{Y_{ \pm} \cos \Delta_{31}}\left[X_{ \pm}^{\text {true }} s_{1}^{2}-X_{ \pm}^{\text {false }} s_{5}^{2}+Z^{\text {true }}-Z^{\text {false }}\right] \\
s_{5} \sin \delta_{5} & =s_{1} \sin \delta_{1}
\end{aligned}
$$


The relation $\cos ^{2} \delta_{5}+\sin ^{2} \delta_{5}=1$ gives the quadratic equation for $s_{5}^{2}$ which results in the similar solution

$$
\begin{gathered}
s_{\mathrm{V}, \mathrm{VI}}^{2}=\frac{1}{\left(X_{ \pm}^{\text {false }}\right)^{2}}\left[X_{ \pm}^{\text {false }}\left\{X_{ \pm}^{\text {true }} s_{1}^{2}+Y_{ \pm} \cos \Delta_{31} s_{1} \cos \delta_{1}+\left(Z^{\text {true }}-Z^{\text {false }}\right)\right\}\right. \\
\left.+\frac{1}{2}\left(Y_{ \pm} \cos \Delta_{31}\right)^{2} \mp d_{ \pm}^{\text {T-oct-intr }} \sqrt{\frac{D_{ \pm}^{\text {T-oct-intr }}}{\left(d_{ \pm}^{\text {T-oct-intr }}\right)^{2}}}\right]
\end{gathered}
$$

where the upper $-($ lower +$)$ sign is for $s_{\mathrm{V}}\left(s_{\mathrm{VI}}\right)$. The functions $D_{ \pm}^{\mathrm{T}-\text { oct-intr }}$ and $d_{ \pm}^{\text {T-oct-intr }}$ are defined by

$$
\begin{aligned}
& D_{ \pm}^{\mathrm{T} \text {-oct-intr }}=\left(Y_{ \pm} \cos \Delta_{31}\right)^{2} \\
\times & {\left[\left(X_{ \pm}^{\text {false }} s_{1} \cos \delta_{1}+\frac{1}{2} Y_{ \pm} \cos \Delta_{31}\right)^{2}+X_{ \pm}^{\text {false }}\left\{\left(Z^{\text {true }}-Z^{\text {false }}\right)-\left(X_{ \pm}^{\text {true }}-X_{ \pm}^{\text {false }}\right) s_{1}^{2}\right\}\right] } \\
& d_{ \pm}^{\text {T-oct-intr }}=\lim _{\theta_{23} \rightarrow \pi / 4} D_{ \pm}^{\mathrm{T}-\text { oct-intr }}=Y_{ \pm} \cos \Delta_{31}\left(X_{ \pm} s_{1} \cos \delta_{1}+\frac{1}{2} Y_{ \pm} \cos \Delta_{31}\right)
\end{aligned}
$$

where $X_{ \pm}$and $Y_{ \pm}$in the last line is meant to be those at $\theta_{23}=\pi / 4$. Once the solutions $s_{\mathrm{V}}$ and $s_{\mathrm{VI}}$ are known one can readily obtain $\delta_{\mathrm{V}}$ and $\delta_{\mathrm{VI}}$ by inserting the $s_{13}$ solutions into (90).

Though, we do not discuss in any detail, it must be obvious that the solutions $\left(s_{\mathrm{VII}}, \delta_{\mathrm{VII}}\right)$ and $\left(s_{\mathrm{VIII}}, \delta_{\mathrm{VIII}}\right)$, the ones with both the $\Delta m_{31}^{2}$-sign and the octant flips, are given by using the solutions obtained in this subsection by the same type of equation as (87): $s_{\mathrm{VII}}=$ $\sqrt{X_{ \pm}^{\text {false }} / X_{\mp}^{\text {false }}} s_{\mathrm{V}}, s_{\mathrm{VIII}}=\sqrt{X_{ \pm}^{\text {false }} / X_{\mp}^{\text {false }}} s_{\mathrm{VI}}, \delta_{\mathrm{VII}}=\pi-\delta_{\mathrm{V}}$, and $\delta_{\mathrm{VIII}}=\pi-\delta_{\mathrm{VI}}$. Alternatively, they can be also obtained by the octant flip mapping, implicitly given in (91) and (90), from $\left(s_{\mathrm{III}}, \delta_{\mathrm{III}}\right)$ and $\left(s_{\mathrm{IV}}, \delta_{\mathrm{IV}}\right)$ obtained in the previous section.

\section{Parameter Degeneracy in Vacuum}

Though it is pedagogically useful to work out the parameter degeneracy in vacuum we just give the results by taking the vacuum limit in the degeneracy solutions obtained for $\mathrm{T}$ conjugate measurement. We need the results in vacuum to define our convention we used to specify unambiguously the solutions for the sign- $\Delta m_{31}^{2}$ degeneracy in Sec. III D. ${ }^{15}$

In fact, it is straightforward to observe that the expressions of the degeneracy solutions in vacuum: They are almost identical to those obtained in this section; The only necessary step is to take the vacuum limit

$$
\begin{aligned}
\lim _{a \rightarrow 0} X_{ \pm} & =X_{v a c} \equiv 4 s_{23}^{2} \sin ^{2} \Delta_{31}, \\
\lim _{a \rightarrow 0} Y_{ \pm} & = \pm Y_{v a c} \equiv \pm 2 \sin 2 \theta_{12} \sin 2 \theta_{23} \Delta_{21} \sin \Delta_{31} \\
\lim _{a \rightarrow 0} Z & =Z_{\text {vac }} \equiv c_{23}^{2} \sin ^{2} 2 \theta_{12},
\end{aligned}
$$

15 Notice that the mass hierarchy does matter in the discussion of parameter degeneracy in vacuum though it might be thought contrary. In fact, it is known that the hierarchy can be determined by measuring the sign of the solar-atmospheric interference term in vacuum 88 . 
in the solutions (80), 81), and (87). Their explicit forms are given in Appendix D of [38]. The degeneracy solutions which involve $\theta_{23}$ octant flip can also be obtained by taking the same limit in the solutions obtained in Sec. VIIC.

\section{PARAMETER DEGENERACY WITH GOLDEN AND SILVER CHANNELS}

We discuss the parameter degeneracy for a given measurement in the $\nu_{e} \rightarrow \nu_{\mu}$ (golden) and the $\nu_{e} \rightarrow \nu_{\tau}$ (silver) channels. The oscillation probability in the former and the latter channels are given by $P^{T}$ in (5) and $P^{S}$ in (10), respectively. We note that detection of $\nu_{\tau}$ requires the energy at least above $\tau$ production threshold, and therefore most probably, neutrino factory would be the appropriate place for the silver channel [71].

\section{A. The intrinsic degeneracy in Golden-Silver measurement}

The intrinsic degeneracy is defined by

$$
\begin{aligned}
& P^{T}-Z=X_{ \pm} s_{1}^{2}+Y_{ \pm} s_{1}\left(\cos \delta_{1} \cos \Delta_{31} \pm \sin \delta_{1} \sin \Delta_{31}\right) \\
& P^{T}-Z=X_{ \pm} s_{2}^{2}+Y_{ \pm} s_{2}\left(\cos \delta_{2} \cos \Delta_{31} \pm \sin \delta_{2} \sin \Delta_{31}\right)
\end{aligned}
$$

and

$$
\begin{aligned}
& P^{S}-\tan ^{2} \theta_{23} Z=\cot ^{2} \theta_{23} X_{ \pm} s_{1}^{2}-Y_{ \pm} s_{1}\left(\cos \delta_{1} \cos \Delta_{31} \pm \sin \delta_{1} \sin \Delta_{31}\right) \\
& P^{S}-\tan ^{2} \theta_{23} Z=\cot ^{2} \theta_{23} X_{ \pm} s_{2}^{2}-Y_{ \pm} s_{2}\left(\cos \delta_{2} \cos \Delta_{31} \pm \sin \delta_{2} \sin \Delta_{31}\right)
\end{aligned}
$$

By subtracting two equations in (94) and (95) we obtain

$$
X_{ \pm}\left(s_{1}^{2}-s_{2}^{2}\right)+Y_{ \pm} S_{I}=0, \quad X_{ \pm}\left(s_{1}^{2}-s_{2}^{2}\right)-\tan ^{2} \theta_{23} Y_{ \pm} S_{I}=0
$$

where

$$
S_{I \pm} \equiv\left[\cos \Delta_{31}\left(s_{1} \cos \delta_{1}-s_{2} \cos \delta_{2}\right) \pm \sin \Delta_{31}\left(s_{1} \sin \delta_{1}-s_{2} \sin \delta_{2}\right)\right]
$$

Then, it follows that $s_{\mathrm{II}}=s_{1}$ (using the label $s_{\mathrm{II}}$ for the intrinsic solution) and $S_{I}=0$ assuming that $Y_{ \pm} \neq 0$. The former result is, of course, expected by the feature of "shrunk ellipse" in the bi-probability plot given in Fig. 22. Using $s_{\mathrm{II}}=s_{1}$ the equation $S_{I}=0$ has a solution, apart from the trivial solution $\delta_{2}=\delta_{1}$, as

$$
\cos \delta_{\mathrm{II}}=\cos \left(\delta_{1} \mp 2 \Delta_{31}\right), \quad \sin \delta_{\mathrm{II}}=-\sin \left(\delta_{1} \mp 2 \Delta_{31}\right)
$$

which implies that

$$
\delta_{\mathrm{II}}=2 \pi-\left(\delta_{1} \mp 2 \Delta_{31}\right) \quad(\bmod .2 \pi) .
$$

The structure of the solutions of $\delta$ should be obvious from the form of the oscillation probabilities in (94) and (95) which is reflected to the feature of shrunk ellipse in Fig. 22, The two degenerate solutions must have the same values of $\cos \left(\delta \mp \Delta_{31}\right)$, and hence $\delta_{\text {II }} \mp \Delta_{31}=2 \pi-\left(\delta_{1} \mp \Delta_{31}\right)$. 


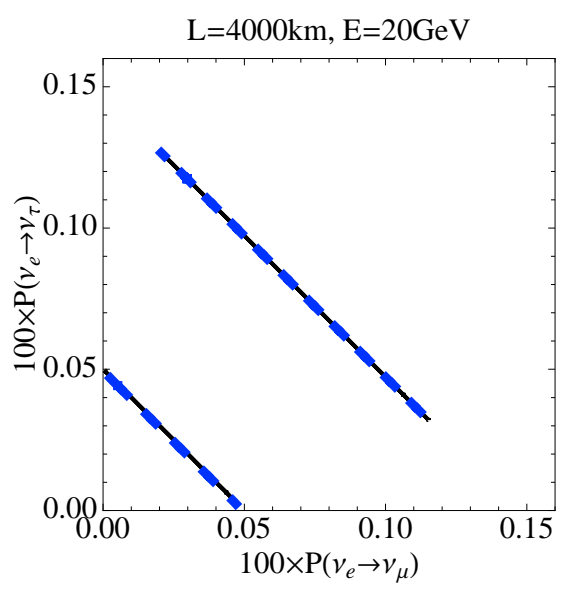

FIG. 22: $\quad P^{T}-P^{S}$ (golden-silver) bi-probability plot for a neutrino factory setting. Black solid line : $\sin ^{2} 2 \theta_{13}=0.004(0.001)$ with normal hierarchy for larger (smaller) oscillation probability. Blue dashed line : $\sin ^{2} 2 \theta_{13}=0.0158(0.0039)$ with inverted hierarchy for lager (smaller) oscillation probability. Notice that black and blue lines are overlapping. $\theta_{23}$ is taken to be 42 degree.

\section{B. The sign- $\Delta m^{2}$ degeneracy in Golden-Silver measurement}

The sign- $\Delta m^{2}$ degeneracy is defined by

$$
\begin{gathered}
P^{T}-Z=X_{ \pm} s_{1}^{2}+Y_{ \pm} s_{1}\left(\cos \delta_{1} \cos \Delta_{31} \pm \sin \delta_{1} \sin \Delta_{31}\right) \\
P^{T}-Z=X_{\mp} s_{3}^{2}+Y_{\mp} s_{3}\left(\cos \delta_{3} \cos \Delta_{31} \mp \sin \delta_{3} \sin \Delta_{31}\right) \\
P^{S}-\tan ^{2} \theta_{23} Z=\cot ^{2} \theta_{23} X_{ \pm} s_{1}^{2}-Y_{ \pm} s_{1}\left(\cos \delta_{1} \cos \Delta_{31} \pm \sin \delta_{1} \sin \Delta_{31}\right) \\
P^{S}-\tan ^{2} \theta_{23} Z=\cot ^{2} \theta_{23} X_{\mp} s_{3}^{2}-Y_{\mp} s_{3}\left(\cos \delta_{3} \cos \Delta_{31} \mp \sin \delta_{3} \sin \Delta_{31}\right)
\end{gathered}
$$

By subtracting two equations in 100 and 101 we obtain

$$
X_{ \pm} s_{1}^{2}-X_{\mp} s_{3}^{2}+S_{S}=0, \quad X_{ \pm} s_{1}^{2}-X_{\mp} s_{3}^{2}-\tan ^{2} \theta_{23} S_{S}=0,
$$

where

$$
S_{S} \equiv \cos \Delta_{31}\left(Y_{ \pm} s_{1} \cos \delta_{1}-Y_{\mp} s_{3} \cos \delta_{3}\right) \pm \sin \Delta_{31}\left(Y_{ \pm} s_{1} \sin \delta_{1}+Y_{\mp} s_{3} \sin \delta_{3}\right) .
$$

Then, 102 implies that the two separate factors both have to vanish:

$$
s_{\mathrm{III}}=\sqrt{\frac{X_{ \pm}}{X_{\mp}}} s_{1}, \quad S_{S}=0,
$$

where we use the notations $\left(s_{\mathrm{III}}, \delta_{\mathrm{III}}\right)$ and $\left(s_{\mathrm{IV}}, \delta_{\mathrm{IV}}\right)$ for the sign- $\Delta m_{31}^{2}$ degeneracy solutions. The former result is in agreement with the symmetry argument given in Sec. IIB. Because $s_{\mathrm{II}}=s_{1}, s_{\mathrm{IV}}=s_{\mathrm{III}}$ holds.

By using the above $s_{3}$ solution and the relation (9) in Sec. II, it is easy to show that the equation $S_{S}=0$ can be converted to the same equation as (98) (after obvious replacement 
of $\delta_{2}$ to $\delta_{3}$ ) apart from the sign change of $\cos \delta_{3}$ term. It means that $\pi-\delta_{3}$ obeys exactly the same equation as (99). Then, we obtain the solutions

$$
\begin{aligned}
& \delta_{\mathrm{III}}=\pi-\delta_{1}, \\
& \delta_{\mathrm{IV}}=\pi-\delta_{\mathrm{II}}=-\pi+\left(\delta_{1} \mp 2 \Delta_{31}\right) \quad(\bmod .2 \pi) .
\end{aligned}
$$

The structure of the solution in one-to-one correspondence to the intrinsic degeneracy solutions is perfectly consistent with the symmetry argument in Sec. II B.

\section{The octant degeneracy in Golden-Silver measurement}

Now, we discuss the $\theta_{23}$ octant degeneracy. We will see that simplicity of the golden-silver setting prevails in it. The $\theta_{23}$ octant degeneracy solutions $\left(s_{5}, \delta_{5}\right)$ satisfy

$$
\begin{aligned}
& P^{T}=X_{ \pm}^{\text {true }} s_{1}^{2}+Y_{ \pm} s_{1}\left(\cos \delta_{1} \cos \Delta_{31} \pm \sin \delta_{1} \sin \Delta_{31}\right)+Z^{\text {true }} \\
& P^{T}=X_{ \pm}^{\text {false }} s_{5}^{2}+Y_{ \pm} s_{5}\left(\cos \delta_{5} \cos \Delta_{31} \pm \sin \delta_{5} \sin \Delta_{31}\right)+Z^{\text {false }}
\end{aligned}
$$

and

$$
\begin{aligned}
& P^{S}=\cot ^{2} \theta_{23} X_{ \pm}^{\text {true }} s_{1}^{2}-Y_{ \pm} s_{1}\left(\cos \delta_{1} \cos \Delta_{31} \pm \sin \delta_{1} \sin \Delta_{31}\right)+\tan ^{2} \theta_{23} Z^{\text {true }} \\
& P^{S}=\tan ^{2} \theta_{23} X_{ \pm}^{\text {false }} s_{5}^{2}-Y_{ \pm} s_{5}\left(\cos \delta_{5} \cos \Delta_{31} \pm \sin \delta_{5} \sin \Delta_{31}\right)+\cot ^{2} \theta_{23} Z^{\text {false }}
\end{aligned}
$$

Using (39), (107) can be written as

$$
\begin{aligned}
& P^{S}=X_{ \pm}^{\text {false }} s_{1}^{2}-Y_{ \pm} s_{1}\left(\cos \delta_{1} \cos \Delta_{31} \pm \sin \delta_{1} \sin \Delta_{31}\right)+Z^{\text {false }} \\
& P^{S}=X_{ \pm}^{\text {true }} s_{5}^{2}-Y_{ \pm} s_{5}\left(\cos \delta_{5} \cos \Delta_{31} \pm \sin \delta_{2} \sin \Delta_{31}\right)+Z^{\text {true }}
\end{aligned}
$$

By subtracting two equations in (106) and (108) with the same octant labels one can easily obtain the solution of $s_{5}$ as $s_{5}^{2}=s_{1}^{2}$ which leads to

$$
s_{\mathrm{V}}=s_{\mathrm{VI}}=s_{1} .
$$

To obtain $\delta_{5}$, we proceed as usual which leads to the result

$$
\cos \left(\delta_{\mathrm{V}} \mp \Delta_{31}\right)=\cos \left(\delta_{1} \mp \Delta_{31}\right)+\frac{1}{s_{1} Y_{ \pm}}\left[\left(X_{ \pm}^{\text {true }}-X_{ \pm}^{\text {false }}\right) s_{1}^{2}+\left(Z^{\text {true }}-Z^{\text {false }}\right)\right] .
$$

As dictated by the general argument, $s_{\mathrm{VI}}$ must be given by $s_{\mathrm{V}}$ as above, and $\delta_{\mathrm{VI}}$ as

$$
\delta_{\mathrm{VI}}=2 \pi-\left(\delta_{V} \mp 2 \Delta_{31}\right) \quad(\bmod .2 \pi),
$$

using (99) because they are the intrinsic degeneracy pair. In fact, it is easy to see that $\delta_{\mathrm{V}}$ and $\delta_{\mathrm{VI}}$ are the two solutions which satisfy $\cos \left(\delta_{\mathrm{V}} \mp \Delta_{31}\right)=\cos \left(\delta_{\mathrm{VI}} \mp \Delta_{31}\right)$ in 1110 .

As in the case of T-conjugate measurement described in Sec. VIIC, the solutions $\left(s_{\mathrm{VII}}, \delta_{\mathrm{VII}}\right)$ and $\left(s_{\mathrm{VII}}, \delta_{\mathrm{VII}}\right)$, the ones with octant as well as the $\Delta m_{31}^{2}$-sign flips, can be obtained from the above ones as

$$
\begin{aligned}
& s_{\mathrm{VII}}=s_{\mathrm{VIII}}=\sqrt{\frac{X_{ \pm}^{\text {false }}}{X_{\mp}^{\text {false }}}} s_{\mathrm{V}} \\
& \delta_{\mathrm{VII}}=\pi-\delta_{\mathrm{V}}, \quad \delta_{\mathrm{VIII}}=\pi-\delta_{\mathrm{VI}} .
\end{aligned}
$$




\section{PARAMETER DEGENERACY IN CPT-CONJUGATE MEASUREMENT}

We discuss in this section the problem of parameter degeneracy in CPT violation measurement. CPT-violation observable was considered to be useful to resolve the mass hierarchy because it gives the probability difference $P-P^{C P T}$ which is largest among the similar quantities [89, 90].

\section{A. The intrinsic degeneracy in CPT-conjugate measurement}

With expression of the oscillation probabilities in (3) and (6), the intrinsic degeneracy solutions $\left(s_{i}, \delta_{i}\right)(\mathrm{i}=1,2)$ in CPT-conjugate measurement are defined with $\nu_{\mu} \rightarrow \nu_{e}$ channel by

$$
\begin{aligned}
& P-Z=X_{ \pm} s_{1}^{2}+Y_{ \pm} s_{1}\left(\cos \delta_{1} \cos \Delta_{31} \mp \sin \delta_{1} \sin \Delta_{31}\right), \\
& P-Z=X_{ \pm} s_{2}^{2}+Y_{ \pm} s_{2}\left(\cos \delta_{2} \cos \Delta_{31} \mp \sin \delta_{2} \sin \Delta_{31}\right)
\end{aligned}
$$

and in CPT-conjugate $\bar{\nu}_{e} \rightarrow \bar{\nu}_{\mu}$ channel by

$$
\begin{aligned}
& P^{C P T}-Z=X_{\mp} s_{1}^{2}-Y_{\mp} s_{1}\left(\cos \delta_{1} \cos \Delta_{31} \mp \sin \delta_{1} \sin \Delta_{31}\right), \\
& P^{C P T}-Z=X_{\mp} s_{2}^{2}-Y_{\mp} s_{2}\left(\cos \delta_{2} \cos \Delta_{31} \mp \sin \delta_{2} \sin \Delta_{31}\right) .
\end{aligned}
$$

By subtracting two equations in 113 and (114) respectively, and subtracting and adding the resultant two equations we obtain, assuming that $C^{(+)} \neq 0$,

$$
S_{I \mp}=0, \quad 2\left(s_{1}^{2}-s_{2}^{2}\right) \pm C^{(-)} S_{I \mp}=0 .
$$

where $C^{( \pm)}$and $S_{I \pm}$ are defined in 17 and $(97)$, respectively. We then obtain $s_{I I}=s_{1}$, that is, the intrinsic degeneracy solution of $\theta_{13}$ for CPT conjugate measurement is identical to the true one, in agreement with the expectation of the bi-probability plot. See Fig. 23. It is obvious that the solution of the first equation (115) is given by

$$
\delta_{\mathrm{II}}=2 \pi-\left(\delta_{1} \pm 2 \Delta_{31}\right) \quad(\bmod .2 \pi) .
$$

\section{B. The sign- $\Delta m^{2}$ degeneracy in CPT-conjugate measurement}

We now discuss the sign- $\Delta m_{31}^{2}$ degeneracy in CPT-conjugate measurement. The true input solution $\left(s_{1}, \delta_{1}\right)$ and the opposite $\Delta m_{31}^{2}$-sign clone solution $\left(s_{3}, \delta_{3}\right)$ satisfy the following equations. In the $\nu_{\mu} \rightarrow \nu_{e}$ channel,

$$
\begin{aligned}
& P-Z=X_{ \pm} s_{1}^{2}+Y_{ \pm} s_{1}\left(\cos \delta_{1} \cos \Delta_{31} \mp \sin \delta_{1} \sin \Delta_{31}\right), \\
& P-Z=X_{\mp} s_{3}^{2}+Y_{\mp} s_{3}\left(\cos \delta_{3} \cos \Delta_{31} \pm \sin \delta_{3} \sin \Delta_{31}\right),
\end{aligned}
$$

and in CPT-conjugate channel

$$
\begin{aligned}
& P^{C P T}-Z=X_{\mp} s_{1}^{2}-Y_{\mp} s_{1}\left(\cos \delta_{1} \cos \Delta_{31} \mp \sin \delta_{1} \sin \Delta_{31}\right) \\
& P^{C P T}-Z=X_{ \pm} s_{3}^{2}-Y_{ \pm} s_{3}\left(\cos \delta_{3} \cos \Delta_{31} \pm \sin \delta_{3} \sin \Delta_{31}\right) .
\end{aligned}
$$




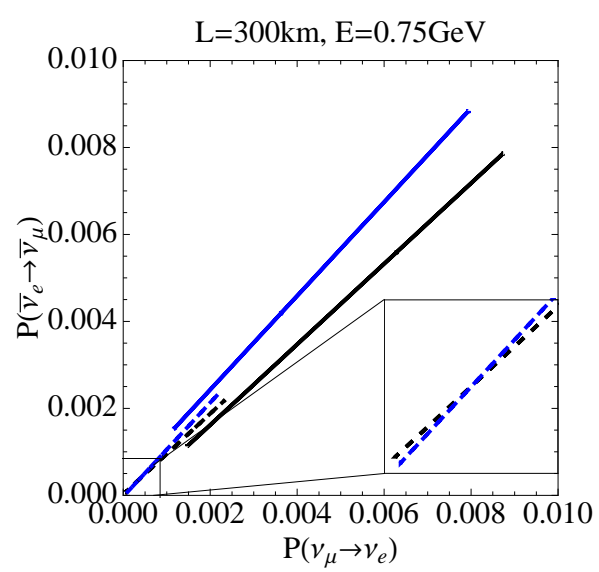

FIG. 23: $P-P^{C P T}$ bi-probability plot. The black and the blue solid (dashed) lines, which correspond respectively to the normal and the inverted hierarchies, are for $\sin ^{2} 2 \theta_{13}=0.01(0.001)$. The region with small probabilities $P \leq 0.0007$ is magnified into the sub-panel to show more clearly the crossing of the two shrunk ellipses in the region.

By subtracting two equations in (117) and (118), respectively, and then subtracting and adding the resultant two equations, we obtain

$$
\begin{array}{r}
\frac{T_{1 \pm}^{C P T}}{C^{(+)}}-s_{3} \cos \delta_{3} \cos \Delta_{31} \mp s_{3} \sin \delta_{3} \sin \Delta_{31}=0, \\
\frac{T_{2 \pm}^{C P T}-2 s_{3}^{2}}{C^{(-)}} \pm s_{3} \cos \delta_{3} \cos \Delta_{31}+s_{3} \sin \delta_{3} \sin \Delta_{31}=0 .
\end{array}
$$

where we have defined

$$
\begin{aligned}
& T_{1 \pm}^{C P T} \equiv \pm E^{(-)} s_{1}^{2}+D^{(+)}\left[s_{1} \cos \delta_{1} \cos \Delta_{31} \mp s_{1} \sin \delta_{1} \sin \Delta_{31}\right] \\
& T_{2 \pm}^{C P T} \equiv E^{(+)} s_{1}^{2} \pm D^{(-)}\left[s_{1} \cos \delta_{1} \cos \Delta_{31} \mp s_{1} \sin \delta_{1} \sin \Delta_{31}\right]
\end{aligned}
$$

where $C^{( \pm)}$is defined in $(17)$, while $D^{( \pm)}$and $E^{( \pm)}$are given in $(27)$. From 119 and $(120)$ it is straightforward to obtain the $s_{3}$ solution:

$$
s_{\mathrm{III}}=s_{\mathrm{IV}}=\frac{1}{\sqrt{2}} \sqrt{T_{2 \pm}^{C P T} \pm\left(\frac{C^{(-)}}{C^{(+)}}\right) T_{1 \pm}^{C P T}}
$$

where the \pm sign is the hierarchy sign. Upon obtaining the $s_{3}$ solution one can readily obtain $\delta_{3}$ by solving $(119)$ for $\cos \left(\delta_{3} \mp \Delta_{31}\right)$. The solutions read

$$
\begin{aligned}
& \delta_{\mathrm{III}}= \pm \Delta_{31}+\arccos \left(\frac{T_{1 \pm}^{C P T}}{C^{(+)} s_{\mathrm{III}}}\right) \quad(\bmod .2 \pi), \\
& \delta_{\mathrm{IV}}=2 \pi-\delta_{\mathrm{III}} \pm 2 \Delta_{31} .
\end{aligned}
$$

One might have suspected, from the feature of the bi-probability plot in Fig. 23, that the sign- $\Delta m_{31}^{2}$ degeneracy solutions exist in a very limited region of small $\theta_{13}$. Therefore, we present in Fig. 24 the region of no solution of the sign- $\Delta m_{31}^{2}$ degeneracy region by the shaded region. 

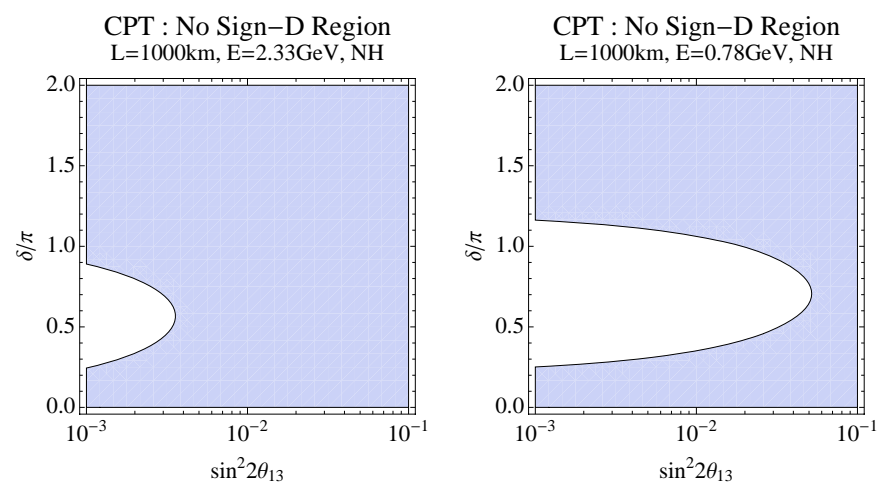

FIG. 24: Depicted as the shaded areas in the $\sin ^{2} 2 \theta_{13}-\delta / \pi$ space are the regions where no sign- $\Delta m_{31}^{2}$ degeneracy solution exists for CPT conjugate measurement for MB1 (left panel) and MB2 (right panel) settings. The true mass hierarchy is taken to be the normal one.

\section{The $\theta_{23}$ octant degeneracy in CPT-conjugate measurement}

The $\theta_{23}$ octant degeneracy is defined by the following two sets of equations:

$$
\begin{gathered}
P=X_{ \pm}^{\text {true }} s_{1}^{2}+Y_{ \pm} s_{1}\left(\cos \delta_{1} \cos \Delta_{31} \mp \sin \delta_{1} \sin \Delta_{31}\right)+Z^{\text {true }}, \\
P=X_{ \pm}^{\text {false }} s_{5}^{2}+Y_{ \pm} s_{5}\left(\cos \delta_{5} \cos \Delta_{31} \mp \sin \delta_{5} \sin \Delta_{31}\right)+Z^{\text {false }} . \\
P^{C P T}=X_{\mp}^{\text {true }} s_{1}^{2}-Y_{\mp} s_{1}\left(\cos \delta_{1} \cos \Delta_{31} \mp \sin \delta_{1} \sin \Delta_{31}\right)+Z^{\text {true }} \\
P^{C P T}=X_{\mp}^{\text {false }} s_{5}^{2}-Y_{\mp} s_{5}\left(\cos \delta_{5} \cos \Delta_{31} \mp \sin \delta_{5} \sin \Delta_{31}\right)+Z^{\text {false }} .
\end{gathered}
$$

Following the similar procedure as before it is not difficult to obtain the equation which involve neither $\delta_{5}$ nor $\delta_{1}$. Then, by using (9) and (39) we obtain

$$
s_{\mathrm{V}}=s_{\mathrm{VI}}=\tan \theta_{23} \sqrt{s_{1}^{2}-\frac{Z^{\text {true }}-Z^{\text {false }}}{\sqrt{X_{ \pm}^{\text {true }} X_{\mp}^{\text {true }}}}} .
$$

Then, the phase $\delta_{5}$ is determined as

$$
s_{\mathrm{V}} \cos \left(\delta_{\mathrm{V}} \pm \Delta_{31}\right)=s_{1} \cos \left(\delta_{1} \pm \Delta_{31}\right)+\left(\frac{1}{Y_{ \pm}}-\frac{1}{Y_{\mp}}\right)\left(Z^{\text {true }}-Z^{\text {false }}\right) .
$$

As in the previous section the intrinsic degeneracy partner $\delta_{\mathrm{VI}}$ is given by using (116) as

$$
\delta_{\mathrm{VI}}=2 \pi-\left(\delta_{V} \pm 2 \Delta_{31}\right) \quad(\bmod .2 \pi) .
$$

As in the previous cases, the solutions with octant as well as the $\Delta m_{31}^{2}$-sign flips, $\left(s_{\mathrm{VII}}, \delta_{\mathrm{VII}}\right)$ and $\left(s_{\mathrm{VII}}, \delta_{\mathrm{VII}}\right)$, are given by the general argument as

$$
\begin{aligned}
& s_{\mathrm{VII}}=s_{\mathrm{VIII}}=\xi_{ \pm}^{\mathrm{CPT}}\left(s_{\mathrm{V}}, \delta_{\mathrm{V}}\right) \\
& \delta_{\mathrm{VII}}=\eta_{ \pm}^{\mathrm{CPT}}\left(s_{\mathrm{V}}, \delta_{\mathrm{V}}\right), \quad \delta_{\mathrm{VIII}}=\eta_{ \pm}^{\mathrm{CPT}}\left(s_{\mathrm{VI}}, \delta_{\mathrm{VI}}\right),
\end{aligned}
$$

where $\xi_{ \pm}^{\mathrm{CPT}}$ and $\eta_{ \pm}^{\mathrm{CPT}}$ are defined in 122 and the first line in 123 , respectively, as a function of $\left(s_{1}, \delta_{1}\right)$. 


\section{CONCLUSION}

In this paper, we have analyzed the problem of parameter degeneracy in various settings, CP-conjugate, T-conjugate, CPT-conjugate measurement, as well as combining the golden and the silver channels. Using the approximate form of the oscillation probabilities obtained by Cervera et al. we have derived, for the first time except for CP-conjugate setting, the exact analytic expressions of the eightfold degeneracy solutions in all these cases assuming $\theta_{23} \neq \pi / 4$. We hope that the simple explicit expressions of the clone solutions nicely fill the "hole" of informations and help understand the nature of the degeneracy. Furthermore, they would prove to be useful if they can be implemented in an analysis codes such as [91, 92. to facilitate the search for fake minima of the $\chi^{2}$. All in all, we expect that such solutions would help in correctly interpreting data to be taken in precision measurement in the future neutrino oscillation experiments.

We have presented a new view of the parameter degeneracy as invariance under the discrete mappings of the flavor mixing parameters including the mass hierarchies. The explicit forms of the mappings can be obtained by the symmetry respected by a pair of the oscillation probabilities alone, (11) in Sec. II B, for the sign- $\Delta m_{31}^{2}$ degeneracy in T-conjugate and the Golden-Silver measurement. In all the other cases, the explicit forms of the mappings are given by the analytic expressions of the degeneracy solutions. Nature of the degeneracy as the intrinsic degeneracy duplicated by the sign of $\Delta m_{31}^{2}$ and $\theta_{23}$ octant is now given its precise meaning as the mapping relations between each pair of degeneracy solutions given in (65). The structure emerged, the one-to-one correspondence between the true and the degeneracy solutions which we call the solution network, is illustrated pictorially in Fig. 4. We have also clarified the relationships between the degeneracy solutions for the given true mass hierarchies, normal or inverted, in Sec. VB.

The explicit analytic expressions of the eightfold degeneracy solutions are used to make plots of the difference between the true and the degeneracy solutions to give an overview of the degeneracy. The features of the degeneracy solutions are so profound, making the true overview of the degeneracy extremely difficult. However, we believe that we have illuminated some of the significant features by taking the three superbeam type settings as well as the one akin to neutrino factory. In particular, the mild energy dependence of the difference between the true and the clone solutions indicate the robustness of the degeneracy against spectrum analysis. These plots illuminate which degeneracy is likely to be difficult to lift, hence it could be useful to design future experiments in preparation of the degeneracy to be met in the measurement. Such precise understanding of the parameter degeneracy would be a definitive help if future precision measurement could be contaminated by new effects outside of the standard three-flavor mixing of neutrinos.

\section{Acknowledgments}

We thank Andrea Donini for the numerous useful informative correspondences. H.M. thanks Renata Zukanovich Funchal and Instituto de Física, Universidade de São Paulo, for the hospitality extended to him during a visit Dec. 2009-Jan. 2010 where part of this work was carried out. This work has been supported in part by KAKENHI, Grant-in-Aid for Scientific Research No. 19340062, and is supported by Grant-in-Aid for JSPS Fellows No. 209677, Japan Society for the Promotion of Science. 


\section{Appendix A: Matter Perturbation Theory of Sign- $\Delta m_{31}^{2}$ Degeneracy}

Here, we present the approximate formulas of the sign- $\Delta m_{31}^{2}$ degeneracy solutions within the framework of matter perturbation theory [77, 78] which assumes $\frac{A}{\Delta_{31}} \ll 1 .{ }^{16}$ As can be seen in (2), the values of the ratio (assuming the matter density and $\Delta m_{31}^{2}$ referenced in the equation) are 0.060, 0.066, and 0.20, respectively, for SB1, MB2, MB1 settings discussed in Sec. VI. Therefore, the condition for validity of matter perturbation theory holds in a good approximation for the former two settings. For MB1 setting the approximation may be modest but we may utilize it for a qualitative discussions.

Having the analytic expressions of the sign- $\Delta m_{31}^{2}$ degeneracy solutions at hand (see Sec. III C), it is straightforward to expand it in terms of the small parameter $\frac{A}{\Delta_{31}}$. We only present the results. To first order in $\frac{A}{\Delta_{31}}$ we obtain the following expressions. For $\theta_{13}$,

$$
\sin ^{2} 2 \theta_{13}^{\mathrm{III}}=\sin ^{2} 2 \theta_{13}^{\mathrm{I}}\left[1+\frac{A}{\Delta_{31}} \frac{4 \sin \delta_{1}\left(\Delta_{31} \cos \Delta_{31}-\sin \Delta_{31}\right)\left(2 s_{1} X_{\mathrm{vac}} \cos \Delta_{31} \pm Y_{\mathrm{vac}} \cos \delta_{1}\right)}{\sin ^{2} \Delta_{31}\left(2 s_{1} X_{\mathrm{vac}} \cos \delta_{1} \pm Y_{\mathrm{vac}} \cos \Delta_{31}\right)}\right]
$$

and for $\delta$,

$$
\begin{aligned}
\cos \delta_{\mathrm{III}}=-\cos \delta_{1} & +4\left(\frac{A}{\Delta_{31}}\right)\left[\frac{\sin \delta_{1}\left(\Delta_{31} \cos \Delta_{31}-\sin \Delta_{31}\right)}{\sin ^{2} \Delta_{31}}\right] \\
& \times\left[\frac{s_{1}^{2} X_{\mathrm{vac}}+Z_{\mathrm{vac}} \cos \left(\delta_{1}-\Delta_{31}\right) \cos \left(\delta_{1}+\Delta_{31}\right) \pm s_{1} Y_{\mathrm{vac}} \cos \delta_{1} \cos \Delta_{31}}{ \pm s_{1} Y_{\mathrm{vac}} \cos \delta_{1}+2 Z_{\mathrm{vac}} \cos \Delta_{31}}\right], \\
\sin \delta_{\mathrm{III}}=\sin \delta_{1} & +4\left(\frac{A}{\Delta_{31}}\right)\left[\frac{\cos \delta_{1}\left(\Delta_{31} \cos \Delta_{31}-\sin \Delta_{31}\right)}{\sin ^{2} \Delta_{31}}\right] \\
& \times\left[\frac{s_{1}^{2} X_{\mathrm{vac}}+Z_{\mathrm{vac}} \cos \left(\delta_{1}-\Delta_{31}\right) \cos \left(\delta_{1}+\Delta_{31}\right) \pm s_{1} Y_{\mathrm{vac}} \cos \delta_{1} \cos \Delta_{31}}{ \pm s_{1} Y_{\mathrm{vac}} \cos \delta_{1}+2 Z_{\mathrm{vac}} \cos \Delta_{31}}\right],
\end{aligned}
$$

where $X_{\text {vac }}$ etc. are defined in Sec. VIID.

\section{Appendix B: Perturbation Theory of $\theta_{23}$ Octant Degeneracy}

In order to understand features of $\theta_{23}$ octant degeneracy it is useful to have a perturbative framework assuming that deviation of $\theta_{23}$ from the maximal is small,

$$
\theta_{23}=\frac{\pi}{4}+\epsilon_{\mathrm{oct}} \quad\left(\epsilon_{\mathrm{oct}} \ll 1\right) .
$$

By expanding the octant degeneracy solution derived in Sec. IV we obtain $\theta_{13}$ to first order in $\epsilon_{\mathrm{oct}}$, as

$$
\begin{aligned}
& \sin ^{2} 2 \theta_{13}^{\mathrm{V}}=\sin ^{2} 2 \theta_{13}^{\mathrm{I}}\left(1+4 \epsilon_{\mathrm{oct}}\right) \\
& +16 \epsilon_{\mathrm{oct}} Z\left[\frac{ \pm 2 s_{1}^{2} \sqrt{X_{ \pm} X_{\mp}} \sin 2 \Delta_{31}+s_{1}\left\{Y_{ \pm} \sin \left(\delta_{1} \pm \Delta_{31}\right)+Y_{\mp} \sin \left(\delta_{1} \mp \Delta_{31}\right)\right\}}{\sqrt{X_{ \pm} X_{\mp}}\left[s_{1}\left\{Y_{ \pm} \sin \left(\delta_{1} \mp \Delta_{31}\right)+Y_{\mp} \sin \left(\delta_{1} \pm \Delta_{31}\right)\right\} \mp 2 Z \sin 2 \Delta_{31}\right]}\right]
\end{aligned}
$$

16 It is known that the matter perturbation theory treatment of the parameter degeneracy gives rise to a transparent view of the degeneracy, which include e.g., decoupling between degeneracies [38, 54, 58]. 
where $X_{ \pm}$etc. implies those evaluated at $\theta_{23}=\pi / 4$. Similarly, we obtain for $\delta$

$$
\begin{aligned}
& \cos \delta_{\mathrm{V}}=\cos \delta_{1}+2 \epsilon_{\mathrm{oct}} \sin \delta_{1} \\
& \times\left[\frac{\left(s_{1}^{2} X_{\mp}-Z\right) Y_{ \pm} \cos \left(\delta_{1} \pm \Delta_{31}\right)+\left(s_{1}^{2} X_{ \pm}-Z\right) Y_{\mp} \cos \left(\delta_{1} \mp \Delta_{31}\right)-2 s_{1} Z\left(X_{ \pm}-X_{\mp}\right)}{s_{1} \sqrt{X_{ \pm} X_{\mp}}\left\{s_{1} Y_{\mp} \sin \left(\delta_{1} \pm \Delta_{31}\right)+s_{1} Y_{ \pm} \sin \left(\delta_{1} \mp \Delta_{31}\right)-2 Z \sin 2 \Delta_{31}\right\}}\right], \\
& \sin \delta_{\mathrm{V}}=\sin \delta_{1}-2 \epsilon_{\mathrm{oct}} \cos \delta_{1} \\
& \quad \times\left[\frac{\left(s_{1}^{2} X_{\mp}-Z\right) Y_{ \pm} \cos \left(\delta_{1} \pm \Delta_{31}\right)+\left(s_{1}^{2} X_{ \pm}-Z\right) Y_{\mp} \cos \left(\delta_{1} \mp \Delta_{31}\right)-2 s_{1} Z\left(X_{ \pm}-X_{\mp}\right)}{s_{1} \sqrt{X_{ \pm} X_{\mp}}\left\{s_{1} Y_{\mp} \sin \left(\delta_{1} \pm \Delta_{31}\right)+s_{1} Y_{ \pm} \sin \left(\delta_{1} \mp \Delta_{31}\right)-2 Z \sin 2 \Delta_{31}\right\}}\right] .
\end{aligned}
$$

[1] Z. Maki, M. Nakagawa and S. Sakata, Prog. Theor. Phys. 28, 870 (1962).

[2] T. Kajita, New J. Phys. 6, 194 (2004).

[3] A. B. McDonald, New J. Phys. 6, 121 (2004) [arXiv:astro-ph/0406253.

[4] K. Inoue, New J. Phys. 6, 147 (2004).

[5] E. Aliu et al. [K2K Collaboration], Phys. Rev. Lett. 94, 081802 (2005) arXiv:hep-ex/0411038]. M. H. Ahn et al. [K2K Collaboration], Phys. Rev. D 74, 072003 (2006) arXiv:hep-ex/0606032.

[6] P. Adamson et al. [MINOS Collaboration], Phys. Rev. Lett. 101, 131802 (2008) arXiv:0806.2237 [hep-ex]].

[7] V. Martemyanov, L. Mikaelyan, V. Sinev, V. Kopeikin and Yu. Kozlov, Phys. Atom. Nucl. 66, 1934 (2003) [Yad. Fiz. 66, 1982 (2003)] arXiv:hep-ex/0211070].

[8] H. Minakata, H. Sugiyama, O. Yasuda, K. Inoue and F. Suekane, Phys. Rev. D 68, 033017 (2003) [Erratum-ibid. D 70, 059901 (2004)] [arXiv:hep-ph/0211111.

[9] F. Ardellier et al. [Double Chooz Collaboration], arXiv:hep-ex/0606025,

[10] X. Guo et al. [Daya Bay Collaboration], arXiv:hep-ex/0701029;

[11] K. K. Joo [RENO Collaboration], Nucl. Phys. Proc. Suppl. 168, 125 (2007).

[12] See also K. Anderson et al., arXiv:hep-ex/0402041.

[13] Y. Itow et al., arXiv:hep-ex/0106019.

For an updated version, see: http://neutrino.kek.jp/jhfnu/loi/loi.v2.030528.pdf

[14] D. Ayres et al. [Nova Collaboration], arXiv:hep-ex/0503053.

[15] M. Kobayashi and T. Maskawa, Prog. Theor. Phys. 49, 652 (1973).

[16] S. Abe et al. [KamLAND Collaboration], Phys. Rev. Lett. 100, 221803 (2008) arXiv:0801.4589 [hep-ex]].

[17] B. Aharmim et al. [SNO Collaboration], arXiv:0910.2984 [nucl-ex].

[18] Y. Ashie et al. [Super-Kamiokande Collaboration], Phys. Rev. Lett. 93, 101801 (2004) arXiv:hep-ex/0404034. Y. Ashie et al. [Super-Kamiokande Collaboration], Phys. Rev. D 71, 112005 (2005) arXiv:hep-ex/0501064.

[19] M. Apollonio et al. [CHOOZ Collaboration], Eur. Phys. J. C 27, 331 (2003) [arXiv:hepex/0301017]; Phys. Lett. B 466, 415 (1999) [arXiv:hep-ex/9907037.

[20] The Palo Verde Collaboration, F. Boehm et al., Phys. Rev. D 64, 112001 (2001) arXiv:hepex/0107009.

[21] M. H. Ahn et al. [K2K Collaboration], Phys. Rev. Lett. 93, 051801 (2004) arXiv:hepex/0402017. 
[22] P. Adamson et al. [MINOS Collaboration], arXiv:0909.4996 [hep-ex].

[23] J. Burguet-Castell, M. B. Gavela, J. J. Gomez-Cadenas, P. Hernandez and O. Mena, Nucl. Phys. B 608, 301 (2001) arXiv:hep-ph/0103258.

[24] H. Minakata and H. Nunokawa, JHEP 0110, 001 (2001) arXiv:hep-ph/0108085.

[25] G. L. Fogli and E. Lisi, Phys. Rev. D 54, 3667 (1996) arXiv:hep-ph/9604415.

[26] H. Minakata and H. Nunokawa, Nucl. Phys. Proc. Suppl. 110, 404 (2002) arXiv:hepph/0111131].

[27] V. Barger, D. Marfatia and K. Whisnant, Phys. Rev. D 65, 073023 (2002) arXiv:hepph/0112119.

[28] T. Kajita, H. Minakata and H. Nunokawa, Phys. Lett. B 528, 245 (2002) arXiv:hepph/0112345].

[29] H. Minakata, H. Nunokawa and S. J. Parke, Phys. Rev. D 66, 093012 (2002) arXiv:hepph/0208163.

[30] A. Cervera, A. Donini, M. B. Gavela, J. J. Gomez Cadenas, P. Hernandez, O. Mena and S. Rigolin, Nucl. Phys. B 579, 17 (2000) [Erratum-ibid. B 593, 731 (2001)] [arXiv:hepph/0002108.

[31] A. Donini, D. Meloni and S. Rigolin, JHEP 0406, 011 (2004) arXiv:hep-ph/0312072.

[32] L. Wolfenstein, Phys. Rev. D 17, 2369 (1978).

[33] J. W. F. Valle, Phys. Lett. B 199 (1987) 432.

[34] M. M. Guzzo, A. Masiero and S. T. Petcov, Phys. Lett. B 260, 154 (1991).

[35] Y. Grossman, Phys. Lett. B 359, 141 (1995) arXiv:hep-ph/9507344.

[36] Z. Berezhiani and A. Rossi, Phys. Lett. B 535, 207 (2002) arXiv:hep-ph/0111137.

[37] H. Minakata, arXiv:0905.1387 [hep-ph].

[38] T. Kikuchi, H. Minakata and S. Uchinami, JHEP 0903, 114 (2009) [arXiv:0809.3312 [hep-ph]].

[39] A. M. Gago, H. Minakata, H. Nunokawa, S. Uchinami and R. Zukanovich Funchal, JHEP 1001, 049 (2010) arXiv:0904.3360 [hep-ph]].

[40] J. Burguet-Castell, M. B. Gavela, J. J. Gomez-Cadenas, P. Hernandez and O. Mena, Nucl. Phys. B 646, 301 (2002) arXiv:hep-ph/0207080.

[41] P. Huber, M. Lindner and W. Winter, Nucl. Phys. B 645, 3 (2002) arXiv:hep-ph/0204352].

[42] P. Huber, M. Lindner and W. Winter, Nucl. Phys. B 654, 3 (2003) |arXiv:hep-ph/0211300].

[43] P. Huber, M. Lindner and W. Winter, JHEP 0505, 020 (2005) arXiv:hep-ph/0412199.

[44] A. Donini, AIP Conf. Proc. 721, 219 (2004) [arXiv:hep-ph/0310014].

[45] D. Autiero et al., Eur. Phys. J. C 33, 243 (2004) arXiv:hep-ph/0305185].

[46] A. Donini, E. Fernandez-Martinez, P. Migliozzi, S. Rigolin and L. Scotto Lavina, Nucl. Phys. B 710, 402 (2005) arXiv:hep-ph/0406132.

[47] J. Burguet-Castell, D. Casper, J. J. Gomez-Cadenas, P. Hernandez and F. Sanchez, Nucl. Phys. B 695, 217 (2004) arXiv:hep-ph/0312068.

[48] O. Mena and S. J. Parke, Phys. Rev. D 70, 093011 (2004) arXiv:hep-ph/0408070.

[49] O. Mena Requejo, S. Palomares-Ruiz and S. Pascoli, Phys. Rev. D 72, 053002 (2005) arXiv:hep-ph/0504015.

[50] O. Mena, S. Palomares-Ruiz and S. Pascoli, Phys. Rev. D 73, 073007 (2006) arXiv:hepph/0510182.

[51] J. E. Campagne, M. Maltoni, M. Mezzetto and T. Schwetz, JHEP 0704, 003 (2007) arXiv:hep$\mathrm{ph} / 0603172$.

[52] D. Beavis et al., arXiv:hep-ex/0205040; M. V. Diwan et al., Phys. Rev. D 68, 012002 (2003) arXiv:hep-ph/0303081. 
[53] M. Ishitsuka, T. Kajita, H. Minakata and H. Nunokawa, Phys. Rev. D 72, 033003 (2005) arXiv:hep-ph/0504026.

[54] T. Kajita, H. Minakata, S. Nakayama and H. Nunokawa, Phys. Rev. D 75, 013006 (2007) arXiv:hep-ph/0609286.

[55] S. Geer, O. Mena and S. Pascoli, Phys. Rev. D 75, 093001 (2007) arXiv:hep-ph/0701258.

[56] A. D. Bross, M. Ellis, S. Geer, O. Mena and S. Pascoli, Phys. Rev. D 77, 093012 (2008) arXiv:0709.3889 [hep-ph]].

[57] V. Barger, M. Dierckxsens, M. Diwan, P. Huber, C. Lewis, D. Marfatia and B. Viren, Phys. Rev. D 74, 073004 (2006) arXiv:hep-ph/0607177.

[58] K. Hiraide, H. Minakata, T. Nakaya, H. Nunokawa, H. Sugiyama, W. J. C. Teves and R. Zukanovich Funchal, Phys. Rev. D 73, 093008 (2006) arXiv:hep-ph/0601258.

[59] O. L. G. Peres and A. Y. Smirnov, Phys. Lett. B 456, 204 (1999) arXiv:hep-ph/9902312]; Nucl. Phys. B 680, 479 (2004) |arXiv:hep-ph/0309312];

[60] M. C. Gonzalez-Garcia, M. Maltoni and A. Y. Smirnov, Phys. Rev. D 70, 093005 (2004) arXiv:hep-ph/0408170.

[61] S. Choubey and P. Roy, Phys. Rev. D 73, 013006 (2006) arXiv:hep-ph/0509197.

[62] M. Shiozawa, T. Kajita, S. Nakayama, Y. Obayashi, and K. Okumura, in Proceedings of the RCCN International Workshop on Sub-dominant Oscillation Effects in Atmospheric Neutrino Experiments, Kashiwa, Japan, Dec. 2004, p.57; T. Kajita, Nucl. Phys. Proc. Suppl. 155, 87 (2006).

[63] P. Huber, M. Maltoni and T. Schwetz, Phys. Rev. D 71, 053006 (2005) arXiv:hepph/0501037].

[64] D. Meloni, Phys. Lett. B 664, 279 (2008) arXiv:0802.0086 [hep-ph]].

[65] E. K. Akhmedov, R. Johansson, M. Lindner, T. Ohlsson and T. Schwetz, JHEP 0404, 078 (2004) arXiv:hep-ph/0402175.

[66] H. Minakata and H. Nunokawa, Phys. Lett. B 495, 369 (2000) arXiv:hep-ph/0004114].

[67] J. Sato, Nucl. Instrum. Meth. A 472, 434 (2001) arXiv:hep-ph/0008056].

[68] B. Richter, arXiv:hep-ph/0008222.

[69] S. Geer, Phys. Rev. D 57, 6989 (1998) [Erratum-ibid. D 59, 039903 (1999)] [arXiv:hepph/9712290];

[70] A. De Rujula, M. B. Gavela and P. Hernandez, Nucl. Phys. B 547, 21 (1999) arXiv:hepph/9811390].

[71] A. Donini, D. Meloni and P. Migliozzi, Nucl. Phys. B 646, 321 (2002) arXiv:hep-ph/0206034].

[72] H. Minakata, H. Nunokawa and S. J. Parke, Phys. Lett. B 537, 249 (2002) arXiv:hepph/0204171.

[73] H. Minakata, Acta Phys. Polon. B 40, 3023 (2009) [arXiv:0910.5545 [hep-ph]].

[74] P. Zucchelli, Phys. Lett. B 532, 166 (2002).

[75] J. Bouchez, M. Lindroos and M. Mezzetto, AIP Conf. Proc. 721, 37 (2004) arXiv:hepex/0310059.

[76] H. Minakata and H. Nunokawa, Nucl. Instrum. Meth. A 503, 218 (2001) arXiv:hepph/0111130.

[77] J. Arafune, M. Koike and J. Sato, Phys. Rev. D 56, 3093 (1997) [Erratum-ibid. D 60, 119905 (1999)] arXiv:hep-ph/9703351].

[78] H. Minakata and H. Nunokawa, Phys. Rev. D 57, 4403 (1998) arXiv:hep-ph/9705208.

[79] S. Uchinami, Dr. of Science Thesis, Tokyo Metropolitan University, online at http://musashi.phys.metro-u.ac.jp/PhD-underscore-Uchinami.pdf 
[80] T. Kobayashi, talk given at 8th TOKUTEI-RCCN Workshop on Neutrinos, November 9, Institute for Cosmic Ray Research, Chiba, Japan (2001), http://www-rccn.icrr.u-tokyo.ac.jp/numeeting/08/04-Kobayashi.pdf, and private communications.

[81] H. Minakata and H. Nunokawa, Phys. Lett. B 413, 369 (1997) arXiv:hep-ph/9706281.

[82] H. Minakata and S. Uchinami, arXiv:1001.4219v1 [hep-ph].

[83] A. Bandyopadhyay et al. [ISS Physics Working Group], Rept. Prog. Phys. 72, 106201 (2009) arXiv:0710.4947 [hep-ph]].

[84] P. Huber and W. Winter, Phys. Rev. D 68, 037301 (2003) arXiv:hep-ph/0301257.

[85] N. Cipriano Ribeiro, H. Minakata, H. Nunokawa, S. Uchinami and R. Zukanovich Funchal, JHEP 0712, 002 (2007) arXiv:0709.1980 [hep-ph]].

[86] J. Kopp, T. Ota and W. Winter, Phys. Rev. D 78, 053007 (2008) [arXiv:0804.2261 [hep-ph]].

[87] T. Abe et al. [ISS Detector Working Group], JINST 4, T05001 (2009) arXiv:0712.4129 [physics.ins-det]].

[88] H. Minakata, H. Nunokawa, S. J. Parke and R. Zukanovich Funchal, Phys. Rev. D 76, 053004 (2007) [Erratum-ibid. D 76, 079901 (2007)] arXiv:hep-ph/0701151.

[89] H. Minakata, H. Nunokawa and S. J. Parke, Phys. Rev. D 68, 013010 (2003) arXiv:hepph/0301210].

[90] A. Jansson, O. Mena, S. J. Parke and N. Saoulidou, Phys. Rev. D 78, 053002 (2008) arXiv:0711.1075 [hep-ph]].

[91] P. Huber, M. Lindner and W. Winter, Comput. Phys. Commun. 167, 195 (2005) arXiv:hepph/0407333.

[92] M. Blennow and E. Fernandez-Martinez, Comput. Phys. Commun. 181, 227 (2010) arXiv:0903.3985 [hep-ph]]. 